\title{
Middle Permian ostracods (Crustacea) from the Guadalupe Mountains, West Texas, USA
}

\author{
Anaëlle TARNAC ${ }^{1}$, Marie-Béatrice FOREL ${ }^{2, *}$, Galina NESTELL ${ }^{3}$, \\ Merlynd NESTELL ${ }^{4} \&$ Sylvie CRASQUIN ${ }^{5}$ \\ ${ }^{1,2,5}$ Centre de Recherches en Paléontologie - Paris, MNHN - SU - CNRS, 8 rue Buffon, \\ 75005 Paris, France. \\ ${ }^{3,4}$ Department of Earth and Environmental Sciences, University of Texas at Arlington, \\ Arlington, TX 76019, USA. \\ "Corresponding author: marie-beatrice.forel@mnhn.fr \\ ${ }^{1}$ Email: tarnac.anaelle@gmail.com \\ ${ }^{3}$ Email: gnestell@uta.edu \\ ${ }^{4}$ Email: nestell@uta.edu \\ ${ }^{5}$ Email: sylvie.crasquin@mnhn.fr \\ ${ }^{1}$ urn:1sid:zoobank.org:author:D9FF2E72-B3F0-4EC7-ADC2-3F04D27E7E1E \\ ${ }^{2}$ urn:lsid:zoobank.org:author:063C1F7E-6D26-48F9-A8B1-2AFA496B5FB8 \\ ${ }^{3}$ urn:lsid:zoobank.org:author:4343960A-89AF-47A7-8418-6B715A7ED791 \\ ${ }^{4}$ urn:1sid:zoobank.org:author:544B3992-191B-4427-B1F7-7A19A9CBCB63 \\ ${ }^{5}$ urn:lsid:zoobank.org:author:6FDDD550-8522-404D-B3A2-D30BFAA86927
}

\begin{abstract}
Middle Permian (Guadalupian) ostracods are described from the Williams Ranch Member of the Cutoff Formation (Roadian) and the McKittrick Canyon Member of the Bell Canyon Formation (Capitanian) from two sections exposed in Culberson County, Guadalupe Mountains, West Texas. Their taxonomy is discussed and adds to the scientific understanding of marine ostracod biodiversity and palaeobiogeography during the Middle Permian. Ostracod assemblages are represented by 51 species of 26 genera and 15 families. Eleven species are newly described: Healdia mckittrickensis Crasquin sp. nov., Healdia cutoffella Crasquin sp. nov., Aurikirkbya guadalupensis Crasquin sp. nov., Hollinella (Hollinella) williamsranchensis Crasquin sp. nov., Geisina culbersonensis Crasquin sp. nov., Paraparchites pecosensis Crasquin sp. nov., Bairdia elcapitanensis Forel sp. nov., Ceratobairdia mescaleroella Forel sp. nov., Ceratobairdia sexagintaduella Forel sp. nov., Ceratobairdia pratti Forel sp. nov., and Denticupachydomella bellcanyonensis Forel sp. nov. The diagnosis of the genus Denticupachydomella is emended. The palaeobiogeographic distribution of the species is analyzed and exemplifies the taxon exchanges between Tethyan and Panthalassic localities in the Permian. The eastern margin of the Palaeo-Tethyan realm is shown to have played a major role in radiation of taxa in the Permian.
\end{abstract}

Keywords. Ostracods, Roadian, Capitanian, Guadalupe Mountains.

Tarnac A., Forel M.-B., Nestell G., Nestell M. \& Crasquin S. 2021. Middle Permian ostracods (Crustacea) from the Guadalupe Mountains, West Texas, USA. European Journal of Taxonomy 770: 1-60.

https://doi.org/10.5852/ejt.2021.770.1499 


\section{Introduction}

The Guadalupian Epoch, Middle Permian, witnessed major global changes, including the formation of the Pangaea supercontinent, the end of the Late Palaeozoic Ice Age, and sea-level changes as well as major biotic events (e.g., Shen et al. 2020 and references therein). Ostracods are millimetre-size crustaceans that are significant components of the meiofauna known from the Early Palaeozoic (e.g., Salas et al. 2007) to the present time. It is now acknowledged that Permian marine ostracod communities went through significant changes up to the end-Permian extinction (see Crasquin \& Forel 2014 and Forel \& Crasquin 2020 for reviews) but their response to the so-called end-Guadalupian mass extinction remains enigmatic. The evolution of meiobenthic biodiversity through this key interval can only be understood by first characterizing the taxonomic structure of Middle Permian communities worldwide. Guadalupian marine ostracods have been reported, for instance, from Greece (Crasquin-Soleau \& Baud 1998), Turkey (Kozur et al. 2000; Crasquin-Soleau et al. 2004), Sultanate of Oman (Crasquin-Soleau et al. 1999), the Russian Platform and Far East in Russia (e.g., Khivintseva 1969; Gramm 1997), Thailand (e.g., Chitnarin et al. 2012, 2017), China (Zazzali et al. 2015), Japan (Ishizaki 1964; Tanaka et al. 2013), Afghanistan (Forel 2018), and North America (e.g., Girty 1908, 1910; Sohn 1954, 1960, 1982).

In North America, Middle Permian ostracods have been described from the Guadalupian outcrops that are widespread in the Delaware Basin: in the Guadalupe, Apache and Glass mountains (Fig. 1). The Guadalupe Mountains are the World stratotype area for the Middle Permian and the Guadalupe Mountains National Park contains the GSSPs for the Roadian, Wordian and Capitanian stages (Glenister et al. 1999). The first appearance of the conodonts Jinogondolella nankingensis (Jin, 1960), J. aserrata (Clark \& Behnken, 1979) and J. postserrata (Behnken, 1975), respectively, define the lower boundary of the Roadian, Wordian and Capitanian stages. The Guadalupian strata present in the Delaware Basin represent various palaeoenvironments from back reef, reef, fore reef to basinal.

The ostracods from the Delaware Basin are known from the Guadalupe and Glass Mountains. In the Guadalupe Mountains, the first ostracods were mentioned by Girty (1908) who described and illustrated (drawings) three species of silicified ostracods: Cythere? sp. from the "dark limestone" of Pine Spring (=Goat Seep Limestone), Bairdia aff. plebeia Reuss, 1854 from basal black limestone (=Bone Spring Limestone of King that includes members of the Cutoff Formation, Lambert et al. 2000) of Guadalupe Point, both from the Guadalupe Mountains, and Argilloecia sp. from the Delaware Mountain Formation of the southern Delaware Mountains. Sohn (1954) included Girty's species Argilloecia sp. in his species Miltonella shupei Sohn, 1950 and reillustrated it in Sohn (1954: pl. 5 fig. 19). Later, Sohn (1960: pl. 1 fig. 22) reillustrated another of Girty's species, Bairdia aff. plebeia, and assigned it to the species Bairdia pecosensis Delo, 1930. According to Sohn (1982: 105), the third of Girty's species Cythere? sp. is "an unidentifiable broken smooth valve on limestone." Sohn (1961a) listed 23 species of ostracods from the Getaway Limestone Member of the Cherry Canyon Formation in the Guadalupe Mountains. Almost all species were identified in open nomenclature, except for six: Amphissites aff. centronotus (Ulrich \& Bassler, 1906), Aurikirkbya aff. wordensis (Hamilton, 1942), Ceratobairdia wordensis (Hamilton, 1942), Healdia? vidriensis Hamilton, 1942, Polytylites digitatus? Sohn, 1954, and Roundyella dorsopapillosa? Sohn, 1954.

In the Glass Mountains, Guadalupian age ostracods have been studied from the Word \# 1 limestone of the Word Formation (Hamilton 1942). According to Cooper \& Grant (1964), this limestone is assigned to the Road Canyon Formation, which is of Roadian age (Wardlaw 2002). Also, ostracods have been described by Sohn $(1950,1954,1962)$ from the upper part of the Leonardian or lower part of the Word Formation (USNM 703c locality that is assigned to the Road Canyon Formation) and the Road Canyon Formation (Sohn 1972). Hamilton (1942) and Sohn $(1950,1954,1962)$ described 29 new species, some of which occur in the material studied herein. 


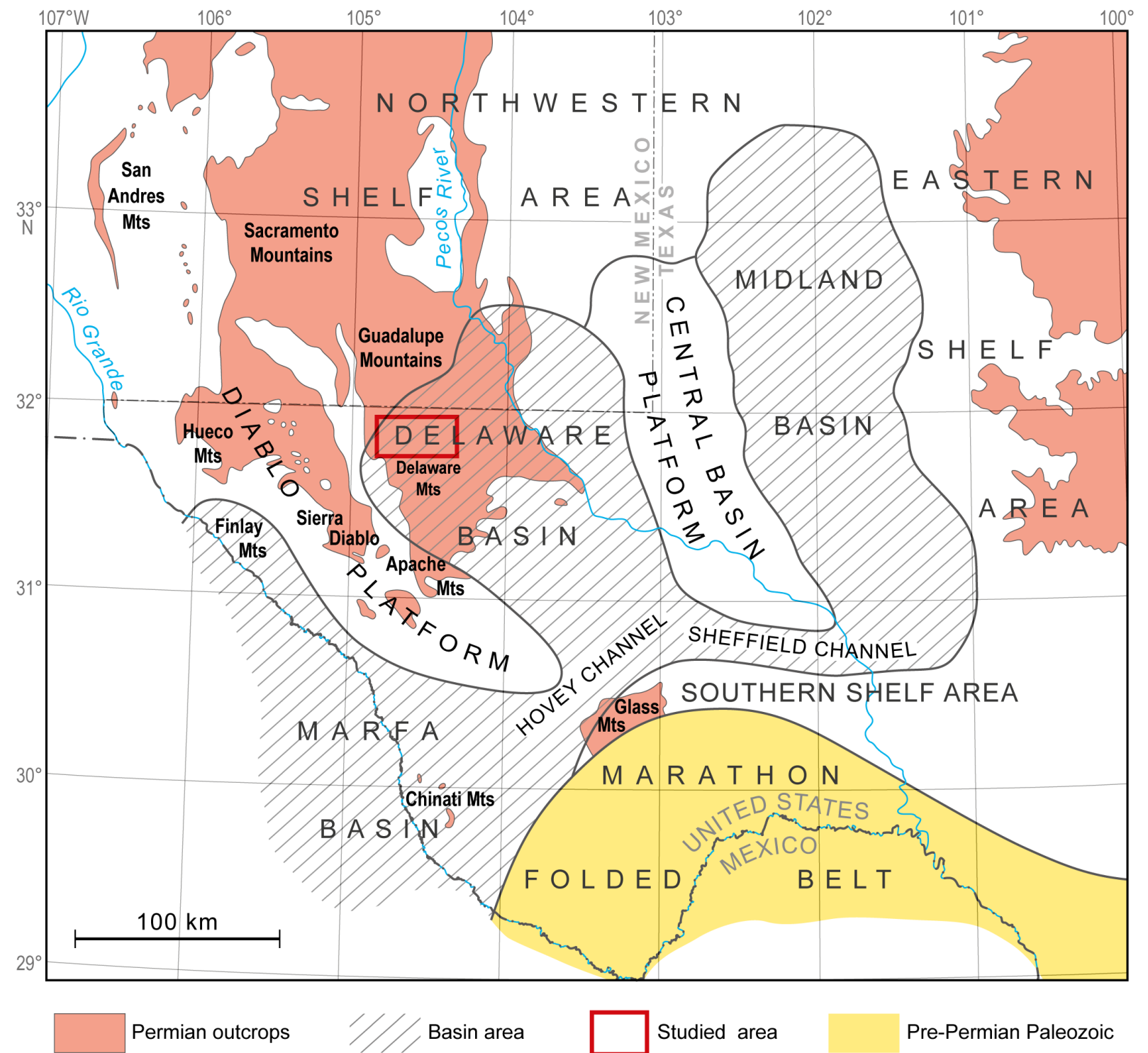

Fig. 1. Major structural features of the Permian Basin region in West Texas, USA showing the location of the Delaware Basin and surrounding mountains (modified from Harris 2000). Black lines indicate the boundaries of provinces of Permian time.

Herein, we analyse ostracod assemblages obtained from two sections in the Guadalupe Mountains area of West Texas: the Quarry section exposing the upper part of the Cutoff Formation (Roadian) and the MKCS1 section exposing the middle part of the Bell Canyon Formation (Capitanian). Ostracods are described and taxonomy is discussed, adding important information to better constraint the Middle Permian biodiversity of ostracod assemblages along the eastern Panthalassa margin. The palaeogeographical links with coeval assemblages are also analysed and provide evidence of TethyanPanthalassic exchanges through the Permian.

\section{Geological setting}

The Guadalupian strata of basinal setting exposed in the Delaware Basin are subdivided into the Cutoff, Brushy Canyon, Cherry Canyon and Bell Canyon Formations (Fig. 2). 


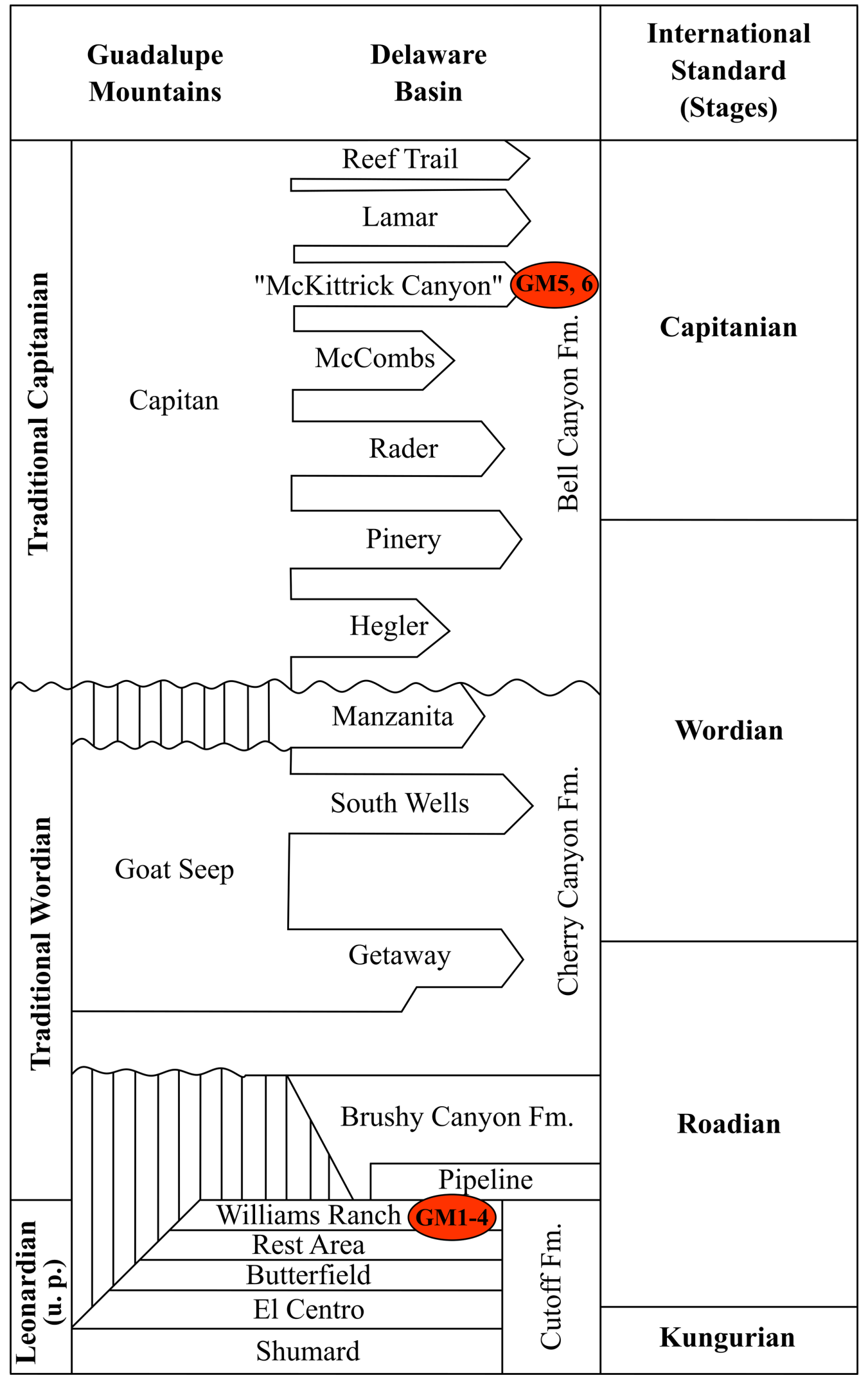

Fig. 2. Stratigraphical units of the Guadalupian in the Delaware Basin area (modified from Wardlaw 2004) and position of the studied Quarry section (samples GM1-GM4) and MKCS1 section (samples GM5, GM6). Abbreviations: u.p. =upper part; Fm. = formation. 


\section{Cutoff Formation}

The history and stratigraphical nomenclature of the Cutoff Formation in the Guadalupe Mountains is discussed in Harris (2000), Lambert et al. (2000), Hurd et al. (2018), and Nestell \& Nestell (2020). The Cutoff Formation is a succession of alternating carbonate and siliciclastic rocks and recently has been subdivided into 5 members (Hurd et al. 2016, 2018): Shumard, El Centro, Butterfield, Rest Area, and Williams Ranch, in ascending order (Fig. 2). The lower boundary of the Roadian is in the middle of the El Centro Member as shown by the first appearance of the conodont J. nankingensis (Glenister et al. 1999; Lambert et al. 2000). The Williams Ranch Member is the uppermost member of the Cutoff Formation that unconformably overlies the Rest Area Member and is unconformably overlain by the Brushy Canyon Formation (Hurd et al. 2018). It ranges from 10 to $47 \mathrm{~m}$ in thickness and consists of deformed soft-sediments, thin-bedded carbonate mudstone, wackstone and packstone.

The Williams Ranch Member recently has been restudied and resampled by Nestell \& Nestell (2020) for the radiolarian analysis in the Quarry section. Besides radiolarians, the studied samples contain conodonts, foraminifers, fish teeth and ostracods, the latter are investigated herein. The Quarry section is located along US Highway 62/180 $3 \mathrm{~km}$ northeast of its junction with Texas Highway 54 (Fig. 3). The strata of the Williams Ranch Member of the Cutoff Formation are exposed as two roadcuts on both sides of the U.S. Highway $62 / 180$. The ostracod fauna is studied from samples taken on the northwestern side of the highway in a small quarry that exposes about $13 \mathrm{~m}$ of limestone beds (Nestell \& Nestell 2020). The bottom of the section is composed of thin-bedded black carbonate mudstone with small black pebbles of packstone with soft-sediment deformation. A zone of debris with exposures in some places of thin- to medium-bedded black carbonate mudstone with lenses of mollusc-bearing packstone occur higher in the section. The packstone lenses contain ammonoids, a very rich assemblage of fish remains, small foraminifers, rare fusulinids, conodonts, diverse radiolarians, scolecodonts, holothurian sclerites, and ostracods (Spinosa et al. 1975; Lambert et al. 2000; Nestell et al. 2015; Nestell \& Nestell 2020).

\section{Bell Canyon Formation}

In the Guadalupe Mountains, the Bell Canyon Formation is subdivided into seven limestone members (in ascending order): Hegler, Pinery, Rader, McCombs, 'McKittrick Canyon' (its status is not formalised yet), Lamar and Reef Trail (Fig. 2). The ostracods were studied from the 'McKittrick Canyon' Member exposed in one section (MKSC1) at the locality GUMO GEO 00252 in the Patterson Hills area (Ivanov et al. 2020). Strata of this locality are represented by thin-bedded limestone about $3 \mathrm{~m}$ thick with several thin beds of debris flow. Eight samples were taken from this locality, but ostracods have been found only in two samples. It is worthy to note that fish remains have recently been described from this locality (Ivanov et al. 2020).

\section{Material and methods}

Six samples processed by diluted acid used for conodont analysis are studied herein (Fig. 2). Four samples from the Williams Ranch Member, Cutoff Formation (Roadian; GM1, GM2, GM3 and GM4) and two samples from the 'McKittrick Canyon' Member, Bell Canyon Formation (Capitanian; samples GM5, GM6) have been studied for ostracod analysis (Fig. 3). All of the recovered specimens of ostracods are silicified. 1662 specimens were mounted on stubs and photographed with SEM. 943 specimens have been identified: they belong to 15 families, 26 genera and 51 species. 23 species are kept in open nomenclature or compared to previously known species.

We follow herein the general ostracod classification of Moore (1961) and Horne et al. (2002).

\section{Material repository}

All type and figured specimens from the Roadian and Capitanian of the Guadalupe Mountains are temporarily deposited in the Micropalaeontology collections of the Muséum national d'histoire naturelle, Paris, France (MNHN) under catalogue numbers MNHN.F.F63365-MNHN.F.F63500 and 


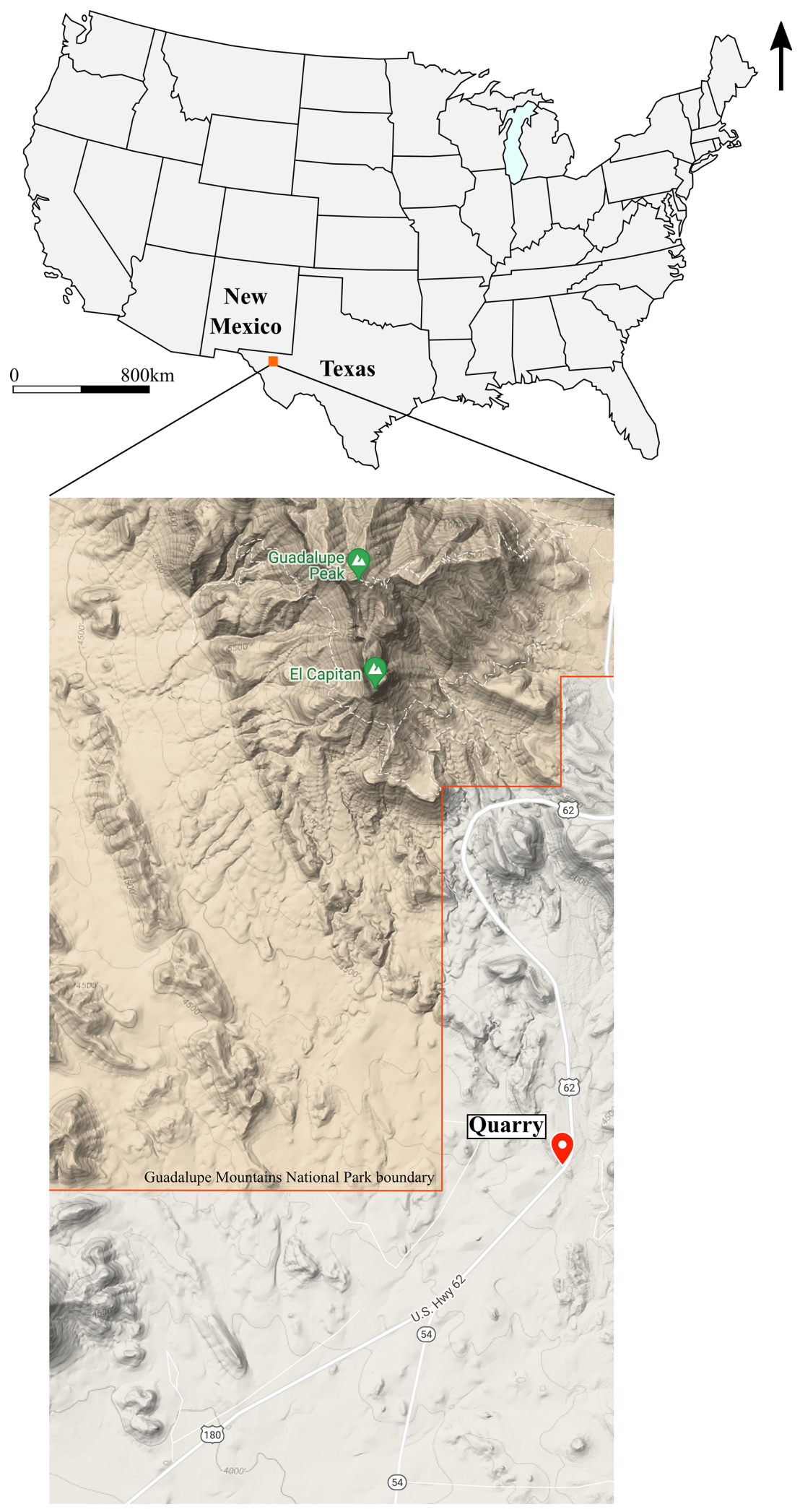

Fig. 3. A. Location of Texas in the USA. B. Location of the studied sections along US Highway $62 / 180$ in the Guadalupe Mountains, West Texas (Google Maps 2021). The orange area shows the Park and its boundary. The section MKSC1 is located within the park but its precise position cannot be disclosed because of park regulations. 
MNHN.F.F63653-MNHN.F.F63660. Individual collection numbers are only provided for type and figured specimens, other mentioned materials are given lot numbers.

Two specimens from the late Capitanian, Middle Permian, Penglaitan section, Guangxi, South China are deposited in the collections of the Sorbonne Universite, Paris, France under catalogue numbers P6M 3778 and P6M 3779.

\section{Anatomical abbreviations}

$\begin{array}{ll}\text { AB } & =\text { anterior border } \\ \mathrm{ACA} & =\text { anterior cardinal angle } \\ \mathrm{ADB} & =\text { antero-dorsal border } \\ \mathrm{AVB} & =\text { antero-ventral border } \\ \mathrm{DB} & =\text { dorsal border } \\ \mathrm{H} & =\text { height } \\ \mathrm{Hmax} & =\text { maximal height } \\ \mathrm{Hmean} & =\text { mean height } \\ \mathrm{L} & =\text { length } \\ \mathrm{L}_{1}, \mathrm{~L}_{2}, \mathrm{~L}_{3} & =\text { lobers } \\ \mathrm{Lmax} & =\text { maximal length } \\ \mathrm{Lmean} & =\text { mean length } \\ \mathrm{LV} & =\text { left valve } \\ \mathrm{PB} & =\text { posterior border } \\ \mathrm{PCA} & =\text { posterior cardinal angle } \\ \mathrm{PDB} & =\text { postero-dorsal border } \\ \mathrm{PVB} & =\text { postero-ventral border } \\ \mathrm{RV} & =\text { right valve } \\ \mathrm{S} & =\text { median sulcus } \\ \mathrm{VB} & =\text { ventral border } \\ \mathrm{W} & =\text { width } \\ \mathrm{Wmax} & =\text { maximal width }\end{array}$

\section{Results}

Systematic palaeontology (M.-B. Forel \& S. Crasquin)

Class Ostracoda Latreille, 1806

Subclass Podocopa Müller, 1894

Order Metacopida Sylvester-Bradley, 1961

Suborder Metacopina Sylvester-Bradley, 1961

Superfamily Healdioidea Harlton, 1933

Family Healdiidae Harlton, 1933

Genus Healdia Roundy, 1926

\section{Type species}

Healdia simplex Roundy, 1926 by original designation.

Healdia mckittrickensis Crasquin sp. nov.

urn:lsid:zoobank.org:act:2454FD9D-BB92-4D01-BD84-11011B2DC72F

Fig. $4 \mathrm{~A}-\mathrm{C}$

\section{Diagnosis}

A species of Healdia with a stocky carapace, a massive overlap of LV on RV, thick posterior spines; a curved ridge extends in front of the spines. 


\section{Etymology}

In reference to the 'McKittrick Canyon' Member, Bell Canyon Formation, Guadalupe Mountains, West Texas, where this species has been discovered.

\section{Material examined}

\section{Holotype}

USA • complete carapace, (Fig. 4A); Guadalupe Mountains, West Texas, 'McKittrick Canyon' Member, Bell Canyon Formation, MKCS1 section, sample GM5; Capitanian, Middle Permian; MNHN.F.F63365.

\section{Paratype}

USA 1 incomplete carapace (DB and PVB of LV are missing), (Fig. 4B); Guadalupe Mountains, West Texas, Williams Ranch Member, Cutoff Formation, Quarry section, sample GM1; Roadian, Middle Permian; MNHN.F.F63366.

\section{Additional material}

USA 11 right valve; Guadalupe Mountains, West Texas, 'McKittrick Canyon' Member, Bell Canyon Formation, MKCS1 section, sample GM5; Capitanian, Middle Permian; MNHN.F F63367 • 3 complete carapaces; Guadalupe Mountains, West Texas, Williams Ranch Member, Cutoff Formation, Quarry section, samples GM1, GM4; Roadian, Middle Permian; MNHN.F.F63473.

\section{Dimensions}

$\mathrm{L}=224-807 \mu \mathrm{m} ; \mathrm{H}=139-489 \mu \mathrm{m}$.

\section{Description}

Carapace stocky, subtriangular to ovoid in lateral view; important overlap of RV by LV with maximum along PDB; dorsal margin of LV regularly arched with Hmax at posterior $1 / 3$ of L; dorsal margin of

Fig. 4 (next page). SEM micrographs of ostracods from the Middle Permian (Roadian, Quarry section, samples GM1, GM2, and GM4; Capitanian, MKCS1 section, samples 4 (GM5) and sample 2 (GM6)) of the Guadalupe Mountains (West Texas, USA). All specimens are temporarily housed in the collections of the Muséum national d'histoire naturelle, Paris, France (MNHN). A-C. Healdia mckittrickensis Crasquin sp. nov. A. Holotype, right lateral view of a carapace, sample 4 (GM5) (MNHN.F.F63365). B. Paratype, right lateral view of a carapace, sample GM1 (MNHN.F.F63366). C. External view of a right valve, sample 4 (GM5) (MNHN.F.F63367). - D-F. Healdia cutoffella Crasquin sp. nov. D. Holotype, right lateral view of a carapace, sample GM4 (MNHN.F.F63368). E. Paratype, external view of a left valve, sample GM1 (MNHN.F.F63369). F. External view of a left valve, sample GM4 (MNHN.F.F63370). - G-I. Sulcella mesopermiana Kozur, 1991. G. External view of a left valve, sample GM1 (MNHN.F.F63371). H. Left lateral view of a carapace, sample 2 (GM6) (MNHN.F.F63372). I. External view of a left valve, sample GM2 (MNHN.F.F63373). - J. Kirkbyidae gen. et sp. indet., right lateral view of a carapace, sample 4 (GM5) (MNHN.F.F63374). - K-L. Amphissites centronotus (Ulrich \& Bassler, 1906). K. External view of a right valve, sample GM1 (MNHN.F.F63375). L. External view of a right valve, sample GM1 (MNHN.F.F63376). - M. Polytylites? sp., external view of a left valve, sample GM4 (MNHN.F.F63377). - N. Kindlella fissiloba Sohn, 1954, external view of a right valve, sample GM1 (MNHN.F.F63378). - O. Kindlella sp., external view of a left valve, sample GM1 (MNHN.F.F63379). - P-Q. Kindlella bellsundi Olempska \& Błaszyk, 1996. P. External view of a right valve, sample 4 (GM5) (MNHN.F.F63380). Q. External view of a right valve, sample 4 (GM5 ) (MNHN.F.F63381) - R. Kirkbya sp., external view of a right valve, sample GM1 (MNHN.F.F63382). Scale bars: $100 \mu \mathrm{m}$. 


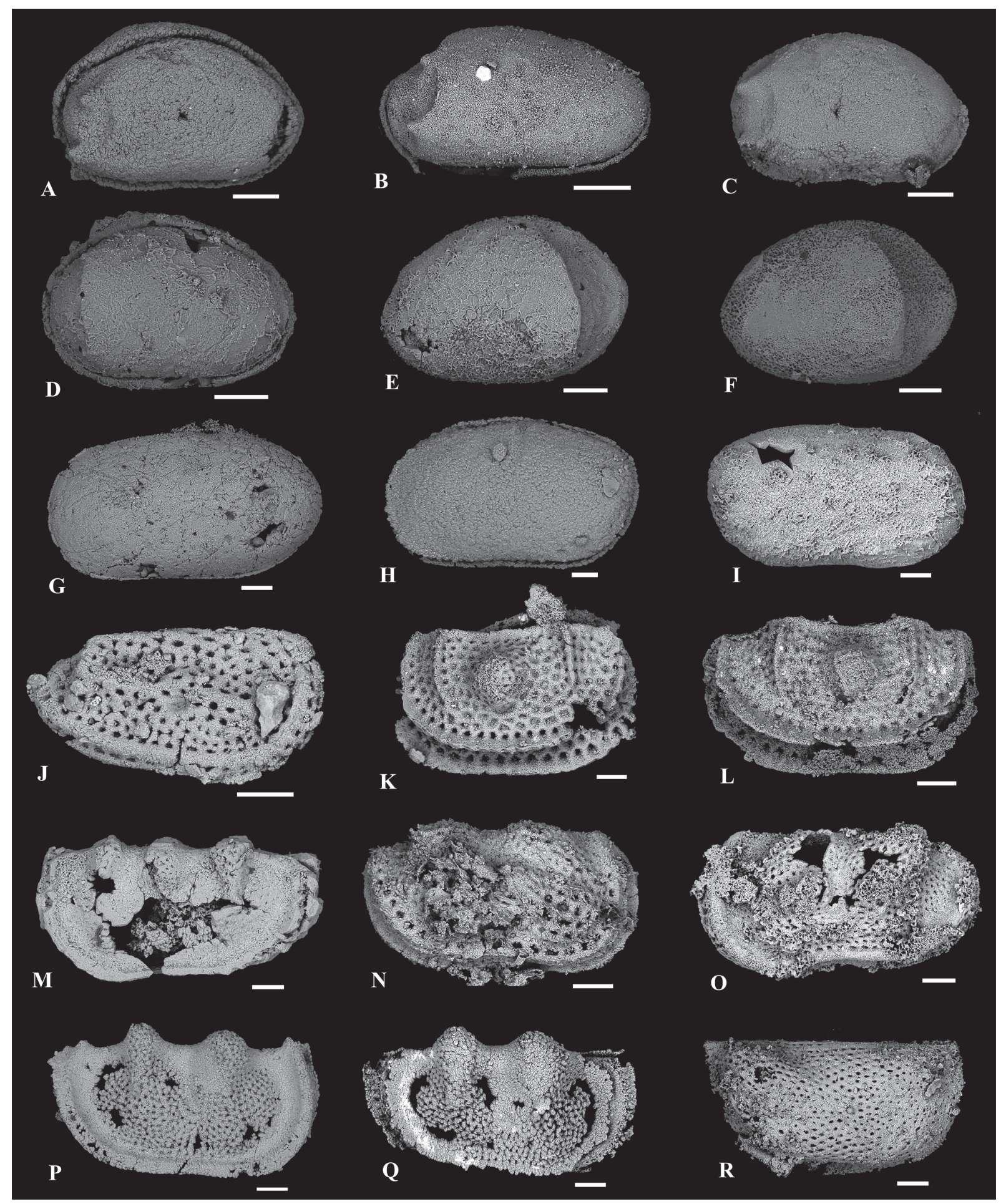


RV with Hmax at mid-L; AB with maximum of convexity located slightly below Hmax; VB slightly concave at both valves; PB large and close to vertical; presence of two posterior spines quite large, their bases are linked by an intra-spines tenuous ridge; in front of the spines, presence of a curved ridge, more developed than the intra-spines one; surface smooth.

\section{Occurrence}

Samples GM1, 4 (GM5), Quarry section, Williams Ranch Member, Cutoff Formation, Roadian; MKCS1 section, sample GM5, 'McKittrick Canyon' Member, Bell Canyon Formation, Capitanian; Middle Permian, Guadalupe Mountains, West Texas, USA (this work).

\section{Remarks}

Healdia mckittrickensis sp. nov. has an unique morphology. The general outline is reminiscent of Healdia subtrangula Kotschetkova, 1959 from the Kazanian (Roadian) of Bashkiria, SW of the Ural Mts (Kotschetkova 1959) but H. mckittrickensis sp. nov. has a less triangular lateral outline with thicker spines.

Healdia cutoffella Crasquin sp. nov. urn:lsid:zoobank.org:act:7615C462-45B0-4D0E-A961-7E469B332767

Figs 4D-F, 5A

\section{Diagnosis}

A species of Healdia with a short and stocky carapace and a posterior ridge more or less vertical without spines, ADB longer than PDB in both valves.

\section{Etymology}

After the Cutoff Formation, Roadian, Middle Permian, West Texas that contains this species.

\section{Material examined}

\section{Holotype}

USA - complete carapace, (Fig. 4D); Guadalupe Mountains, West Texas, Williams Ranch Member, Cutoff Formation, Quarry section, sample GM4; Roadian, Middle Permian; MNHN.F.F63368.

\section{Paratype}

USA - 1 complete carapace, (Fig. 4E); Guadalupe Mountains, West Texas, Williams Ranch Member, Cutoff Formation, Quarry section, sample GM1; Roadian, Middle Permian; MNHN.F.F63369.

\section{Additional material}

USA • 1 left valve, (Fig. 4F); Guadalupe Mountains, West Texas, Williams Ranch Member, Cutoff Formation, Quarry section, sample GM4; Roadian, Middle Permian; MNHN.F.F63370 • 10 complete carapaces, 32 valves; Guadalupe Mountains, West Texas, Williams Ranch Member, Cutoff Formation, Quarry section, samples GM1, GM4; Roadian, Middle Permian; MNHN.F.F63474.

\section{Dimensions}

$\mathrm{L}=452-667 \mu \mathrm{m} ; \mathrm{H}=300-448 \mu \mathrm{m}$ (Fig. 5A)

\section{Description}

Short and stocky carapace, egg-shaped in lateral view; LV strongly overlaps RV all around the carapace; ADB longer than PDB at both valves; $\mathrm{AB}$ large with maximum of curvature located around mid- $\mathrm{H}$; 

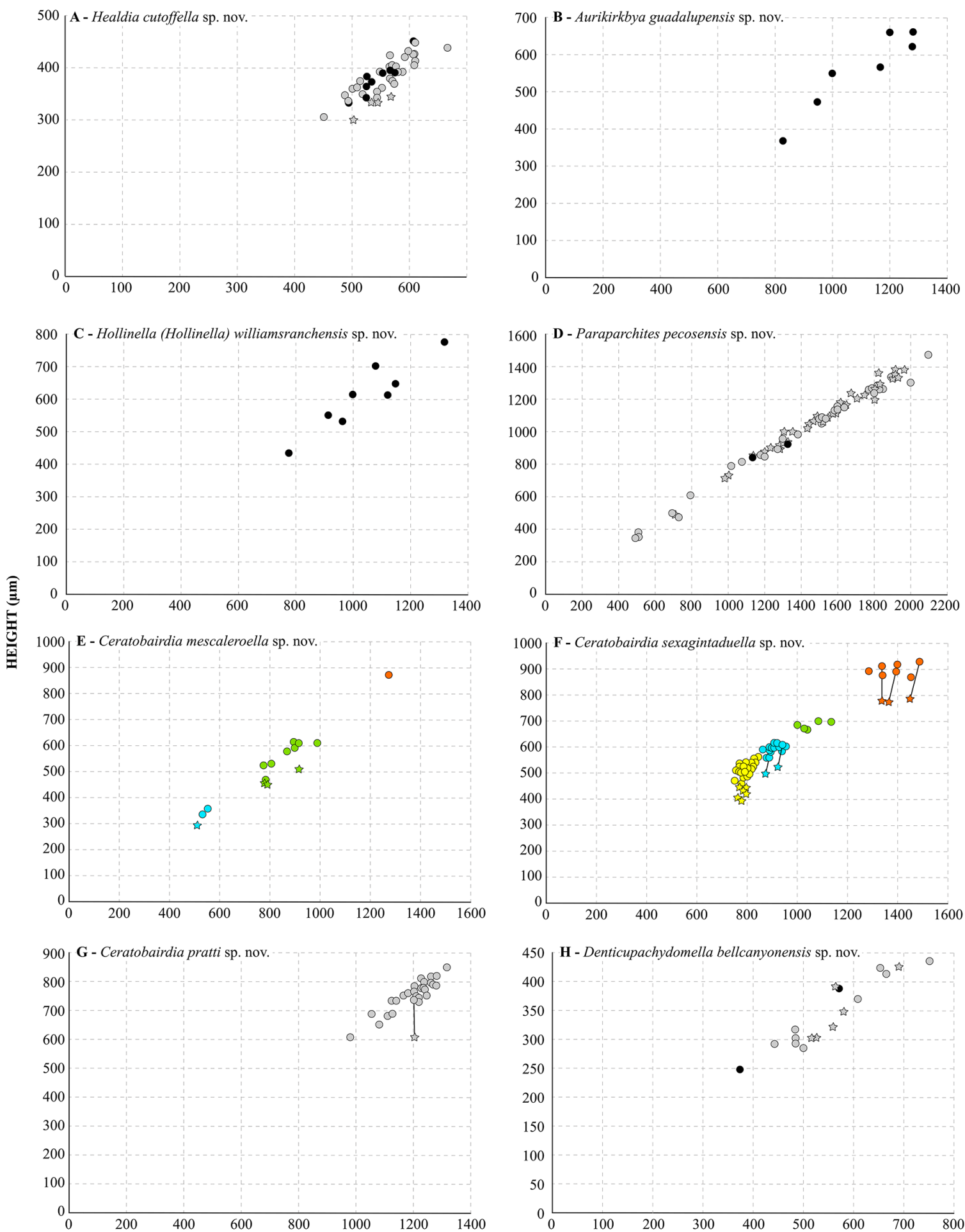

$$
\begin{array}{|ll|}
\hline & \text { carapaces } \\
\text { is right valves } \\
0 & \text { left valves } \\
0 & \text { adults } \\
0 & \text { A-1 } \\
0 & \text { A-2 } \\
0 & \text { A-3 }
\end{array}
$$

Fig. 5. Height/length scatter plots of species from the Middle Permian (Roadian, Capitanian) of the Guadalupe Mountains (West Texas, USA). A-1, A-2, A-3. Three last juvenile stages before adult. 
$\mathrm{PB}$ quite equivalent to $\mathrm{AB}$; presence of a posterior vertical to slightly tilted ridge, without spines; carapace laterally compressed posterior to ridge, more strongly at LV.

\section{Occurrence}

Samples GM1, GM4, Quarry section, Williams Ranch Member, Cutoff Formation, Roadian; sample 4 (GM5), MKCS1 section, 'McKittrick Canyon' Member, Bell Canyon Formation, Capitanian; Middle Permian, Guadalupe Mountains, West Texas, USA (this work).

\section{Remarks}

Healdia cutoffella sp. nov. is very similar to Healdia simplissima Harlton, 1933 from the Pennsylvanian of Texas and Oklahoma (Harlton 1933; Hoare \& Merrill 2004). Both species have the same ridge without spines, equivalent $\mathrm{AB}$ and $\mathrm{PB}$, but mainly differ by $\mathrm{PB}$ being longer than $\mathrm{AB}$ in $H$. simplissima (herein $\mathrm{AB}$ is longer than $\mathrm{PB})$.

Order Platycopida Sars, 1866

Family Cavellinidae Egorov, 1950

Genus Sulcella Coryell \& Sample, 1932

\section{Type species}

Sulcella sulcata Coryell \& Sample, 1932 by original designation.

Sulcella mesopermiana Kozur, 1991

Fig. 4G-I

Sulcella mesopermiana Kozur, 1991: 22, pl. 5 figs 3-4.

Sulcella mesopermiana - Crasquin-Soleau \& Baud 1998: 139, pl. 3 figs 3, 5, 6, 9. - Zazzali et al. 2015: 306, fig. 17a. - Chitnarin et al. 2017: 681, figs 24o-r.

\section{Material examined}

USA - 1 left valve; Guadalupe Mountains, West Texas, Williams Ranch Member, Cutoff Formation, Quarry section, sample GM1; Roadian, Middle Permian; MNHN.F.F63371 • 1 complete carapace; Guadalupe Mountains, West Texas, 'McKittrick Canyon' Member, Bell Canyon Formation, MKCS1 section, sample 2 (GM6); Capitanian, Middle Permian; MNHN.F.F63372 • 1 left valve; Guadalupe Mountains, West Texas, Williams Ranch Member, Cutoff Formation, Quarry section, sample GM2; Roadian, Middle Permian; MNHN.F.F63373 - 3 complete carapaces, 10 valves; Guadalupe Mountains, West Texas, Williams Ranch Member, Cutoff Formation, Quarry section, samples GM1, GM3, GM4; Roadian; MNHN.F.F63653 2 complete carapaces, 7 valves; same locality as for preceding; 'McKittrick Canyon' Member, Bell Canyon Formation, MKCS1 section, samples 4 (GM5) and 2 (GM6); Capitanian, Middle Permian; MNHN.F.F63475.

\section{Occurrence}

Bükk Mountains, Hungary, Capitanian-Wuchiapingian, Middle-Late Permian (Kozur 1991); Hydra Island, Greece, Capitanian-Wuchiapingian, Middle-Late Permian (Crasquin-Soleau \& Baud 1998); Chaotian section, Sichuan, South China, Wuchiapingian, Late Permian (Zazzali et al. 2015); Pha Nok Khao Formation, Khao Kana section, Phetchabun Province, central Thailand, Sakmarian-Artinskian, Early Permian (Chitnarin et al. 2017); Quarry section, samples GM1, GM3, GM4, Williams Ranch Member, Cutoff Formation, Roadian; MKCS1 section, samples 4 (GM5) and 2 (GM6), 'McKittrick Canyon' Member, Bell Canyon Formation, Capitanian; Guadalupe Mountains, West Texas, USA, Middle Permian (this work). 
Order Palaeocopida Henningsmoen, 1953

Suborder Kloedenellocopina Scott, 1961

Superfamily Kirkbyoidea Ulrich \& Bassler, 1906

Kirkbyidae gen. et sp. indet.

Fig. 4J

\section{Material examined}

USA 11 complete carapace; Guadalupe Mountains, West Texas, 'McKittrick Canyon' Member, Bell Canyon Formation, MKCS1 section, sample 4 (GM5); Capitanian, Middle Permian; MNHN.F.F63374.

\section{Remarks}

This specimen shows a clear oval kirkbyan pit in the middle part of the valve. The right valve has $\mathrm{PB}$ and $\mathrm{AB}$ with a different radius of curvature, whereas they are quite equivalent in Kirkbyidae. Herein, the PB has a very small radius of curvature and $\mathrm{AB}$ a large one. The ornamentation is coarse.

\section{Occurrence}

Sample 4 (GM5), MKCS1 section, 'McKittrick Canyon' Member, Bell Canyon Formation, Guadalupe Mountains, West Texas, USA, Capitanian, Middle Permian (this work).

Family Amphissitidae Knight, 1928

Genus Amphissites Girty, 1910

\section{Type species}

Amphissites rugosus Girty, 1910 by original designation.

Amphissites centronotus (Ulrich \& Bassler, 1906)

Fig. 4K-L

Kirkbya centronota Ulrich \& Bassler 1906: 159, pl. 11, figs 16, 17.

Amphissites centronota - Harlton 1927: 207, pl. 32 figs 10a-b (new combination). — Knight 1928: 259, pl. 32 figs 6a-e. - Sohn 1961b: 118-119, pl. 7 figs 8-10. - Kellett 1933: pl. 95, pl. 16 figs 16-22. — Ishizaki 1964: pl. 17 figs 4-5. — Thompson \& Shaver 1964: fig. 2. — Schneider 1966: 110. — Shaver \& Smith 1974: 35, pl. 1 figs 1-5. — Sanchez de Posada 1977: 416, pl. 2 figs 9-11. — Shi 1982: 301, pl. 1 figs 4-6. — Kozur 1991: pl.1 fig. 1. — Zhang \& Liang 1987: 301, pl. 2 fig. 5. — Burke 1985: fig. 4.12. — Jones 1988: 260, text fig. 1a, pl. 1 figs 1-4, 7; 1989: 44, pl. 10, figs 1a-2d, text fig. 19b. - Melnyk \& Maddocks 1988: pl. 2 fig. 9. — Christopher et al. 1990: 979, figs 4.164.20. - Fohrer 1991: 22, pl. 7 figs 1-3; 1997: 114-115, pl. 10, figs 7-8. — Becker \& Wang 1992: 12, pl. 1 fig. 5. - Crasquin-Soleau \& Orchard 1994: pl. 3 fig. 2. - Crasquin-Soleau 1997: 51, pl. 2 figs 9-11. - Becker 1997: pl. 3 figs 1-2. - Ferdinando 2001: 43-44, pl. 1 figs 29-30, pl. 2 figs 1-8. Amphissites (Amphissites) centronotus - Olempska 1999: 406, figs 14a-b.

\section{Material examined}

USA 1 right valve; Guadalupe Mountains, West Texas, Williams Ranch Member, Cutoff Formation, Quarry section, sample GM1; Roadian, Middle Permian; MNHN.F.F63375 • 1 right valve; same locality as preceding; Roadian, Middle Permian; MNHN.F.F63376 • 24 valves; Guadalupe Mountains, West 
Texas, Williams Ranch Member, Cutoff Formation, Quarry section, samples GM1, GM4; Roadian, Middle Permian; MNHN.F.F63476.

\section{Occurrence}

Kansas, USA, Carboniferous (Ulrich \& Bassler 1906); Oklahoma, USA, Pennsylvanian (Harlton 1927); Texas, USA, Pennsylvanian (Coryell \& Billings 1932); USA, Gzhelian, Pennsylvanian, SakmarianArtinskian, Early Permian (Kellett 1933); Kansas, USA, Carboniferous-Permian (Sohn 1961b); Japan, Capitanian, Middle Permian (Ishizaki 1964); Illinois, USA, Early Pennsylvanian (Thompson \& Shaver 1964); Russian Platform, Late Permian (Schneider 1966); Illinois, USA, Pennsylvanian (Shaver \& Smith 1974); Asturias, Spain, Late Carboniferous (Sanchez de Posada 1977); Ohio, USA, Pennsylvanian (Burke 1985); Bükk Mountains, Hungary, Moscovian, Late Carboniferous (Kozur 1991); Henan Province, East China, Late Carboniferous (Zhang \& Liang 1987); Texas, USA, Pennsylvanian-Kungurian (Melnyk \& Maddocks 1988); Bonaparte Basin, Australia, Early Carboniferous (Jones 1988, 1989); Appalachian Basin, USA, Pennsylvanian (Christopher et al. 1990); South China, Tournaisian, Early Carboniferous (Becker \& Wang 1992; Olempska 1999); British Columbia, Canada, Sakmarian-Artinskian, Early Permian (Crasquin-Soleau \& Orchard 1994; Crasquin-Soleau 1997); Cantabrian Mountains, Spain, Pennsylvanian (Becker 1997); Carnic Alps, Austria, Asselian, Early Permian (Fohrer 1997); Perth Basin, Australia, Sakmarian, Early Permian (Ferdinando 2001); West Texas, USA, Middle Permian, Roadian, Quarry section, samples GM1, GM4, Williams Ranch Member, Cutoff Formation, Guadalupe Mountains (this work).

Family Kelletinidae Sohn, 1954

Genus Kindlella Sohn, 1954

\section{Type species}

Kindlella fissiloba Sohn, 1954 by original designation.

Kindlella fissiloba Sohn, 1954

Fig. 4N

Kindlella fissiloba Sohn, 1954: 16-17, pl. 2 fig. 12, pl. 3 figs 22-27, pl. 4 fig. 13.

\section{Material examined}

USA 1 right valve; Guadalupe Mountains, West Texas, Williams Ranch Member, Cutoff Formation, Quarry section, sample GM1; Roadian, Middle Permian; MNHN.F.F63378 - 3 valves; Guadalupe Mountains, West Texas, Williams Ranch Member, Cutoff Formation, Quarry section, samples GM1, GM4; Roadian, Middle Permian; MNHN.F.F63477.

\section{Occurrence}

Texas, USA, Roadian, Middle Permian (Sohn 1954); samples GM1, GM4, Quarry section, Williams Ranch Member, Cutoff Formation, Guadalupe Mountains, West Texas, USA, Roadian, Middle Permian (this work).

Kindlella bellsundi Olempska \& Błaszyk, 1996

Fig. $4 \mathrm{P}-\mathrm{Q}$

Kindlella bellsundi Olempska \& Błaszyk, 1996: 11-12, pl. 2 figs 3-4, pl. 3 figs 1-4. 


\section{Material examined}

USA 11 right valve; Guadalupe Mountains, West Texas, 'McKittrick Canyon' Member, Bell Canyon Formation, MKCS1 section, sample 4 (GM5); Capitanian, Middle Permian; MNHN.F.F63380 • 1 right valve; same locality as for preceding; Capitanian, Middle Permian; MNHN.F.F63381.

\section{Remarks}

The specimens have a transitional morphology between Kindlella Sohn, 1954 and Kellettina Swartz, 1936. They have the carapace outline and characters of Kindlella and lobes overpassing the DB as in Kelletina.

\section{Occurrence}

West Spitzbergen, Norway, Wuchiapingian, Late Permian (Olempska \& Błaszyk 1996); sample 4 (GM5), MKCS1 section, 'McKittrick Canyon' Member, Bell Canyon Formation, Guadalupe Mountains, West Texas, USA, Capitanian, Middle Permian (this work).

\section{Kindlella sp.}

Fig. $4 \mathrm{O}$

\section{Material examined}

USA 1 right valve; Guadalupe Mountains, West Texas, Quarry section, sample GM1, Williams Ranch Member, Cutoff Formation; Roadian, Middle Permian (this work); MNHN.F.F63379.

\section{Occurrence}

Sample GM1, Quarry section, Williams Ranch Member, Cutoff Formation, Guadalupe Mountains, West Texas, USA, Roadian, Middle Permian (this work).

Family Kirkbyidae Ulrich \& Bassler, 1906

Genus Kirkbya Jones, 1859

\section{Type species}

Dithyrocaris permiana Jones, 1850 subsequently designated by Jones (1859).

\section{Kirkbya sp.}

Fig. 4R

\section{Material examined}

USA 1 right valve; Guadalupe Mountains, West Texas, Williams Ranch Member, Cutoff Formation, Quarry section, sample GM1; Roadian, Middle Permian; MNHN.F.F63382.

\section{Remarks}

The general outline of Kirkbya sp. is close to that of Kirkbya permiana (Jones, 1850) in Woszczyńska, 1987 from the Late Permian of Poland (Woszczyńska 1987) and to Kirkbya bendensis Harlton, 1933 from the Pennsylvanian of USA (Harlton 1933) but the ornamentation in the Texan specimens is very different by a very fine reticulation. 


\section{Occurrence}

Sample GM1, Quarry section, Williams Ranch Member, Cutoff Formation, Guadalupe Mountains, West Texas, USA, Roadian, Middle Permian (this work).

Genus Aurikirkbya Sohn, 1950

\section{Type species}

Kindlella wordensis Hamilton, 1942 subsequently designated by Sohn (1950).

Aurikirkbya guadalupensis Crasquin sp. nov.

urn:lsid:zoobank.org:act:FD8EB2E1-3BDB-4A75-ADA2-4FB287EB27AA

Figs $5 \mathrm{~B}, 6 \mathrm{~A}-\mathrm{B}$

\section{Diagnosis}

A species of Aurikirkbya with an additional ventral ridge parallel to the adventral ridge, extending from mid- $\mathrm{H}$ in the anterior part to mid- $\mathrm{H}$ in the posterior part of the carapace.

\section{Etymology}

After the Guadalupe Mountains, West Texas, USA, where this species has been discovered.

\section{Material examined}

\section{Holotype}

USA - complete carapace, (Fig. 6A); Guadalupe Mountains, West Texas, Williams Ranch Member, Cutoff Formation, Quarry section, sample GM2; Roadian, Middle Permian; MNHN.F.F63383.

Fig. 6 (next page). SEM micrographs of ostracods from the Middle Permian (Roadian, Quarry section, samples GM1, GM2, and GM4; Capitanian, MKCS1 section, samples 4 (GM5) and 2 (GM6)) of the Guadalupe Mountains (West Texas, USA). All specimens are temporarily housed in the collections of the Muséum national d'histoire naturelle, Paris, France (MNHN). A-B. Aurikirkbya guadalupensis Crasquin sp. nov. A. Holotype, left lateral view of a carapace, sample GM2 (MNHN.F.F63383). B. Paratype, left lateral view of a carapace, sample GM1 (MNHN.F.F63384). - C, E. Aurikirkbya wordensis (Hamilton, 1942). C. External view of a left valve, sample 2 (GM6) (MNHN.F.F63385). E. External view of a left valve, sample GM4 (MNHN.F.F63387). - D. Kirkbyella (Berdanella) cf. quadrata Croneis \& Gutke, 1939, external view of a right valve, sample 4 (GM5) (MNHN.F.F63386). - F-H. Roundyella lebaensis Krömmelbein, 1958. F. External view of a left valve, sample GM4 (MNHN.F.F63388). G. External view of a right valve, sample GM4 (MNHN.F.F63389). H. External view of a right valve, sample GM1 (MNHN.F.F63390). - I-L. Hollinella (Hollinella) williamsranchensis Crasquin sp. nov. I. Holotype, external view of a right valve, sample GM1 (MNHN.F.F63391). J. External view of a right valve, sample GM1 (MNHN.F.F63392). K. Paratype, external view of a left valve, sample GM1 (MNHN.F.F63393). L. External view of a left valve, sample GM4 (MNHN.F.F63394). - M. Glyptopleura sp., external view of a right valve, sample GM1 (MNHN.F.F63395). - N-P. Geisina culbersonensis Crasquin sp. nov. N. Holotype, external view of a right valve, sample GM1 (MNHN.F.F63396). O. Paratype, external view of a right valve, sample GM1 (MNHN.F.F63397). P. External view of a left valve, sample GM2 (MNHN.F.F63398). - Q-R. Miltonella cf. shupei Sohn, 1950. Q. External view of a left valve, sample 4 (GM5) (MNHN.F.F63399). R. External view of a left valve, sample 4 (GM5) (MNHN.F.F63400). Scale bars: $100 \mu \mathrm{m}$. 


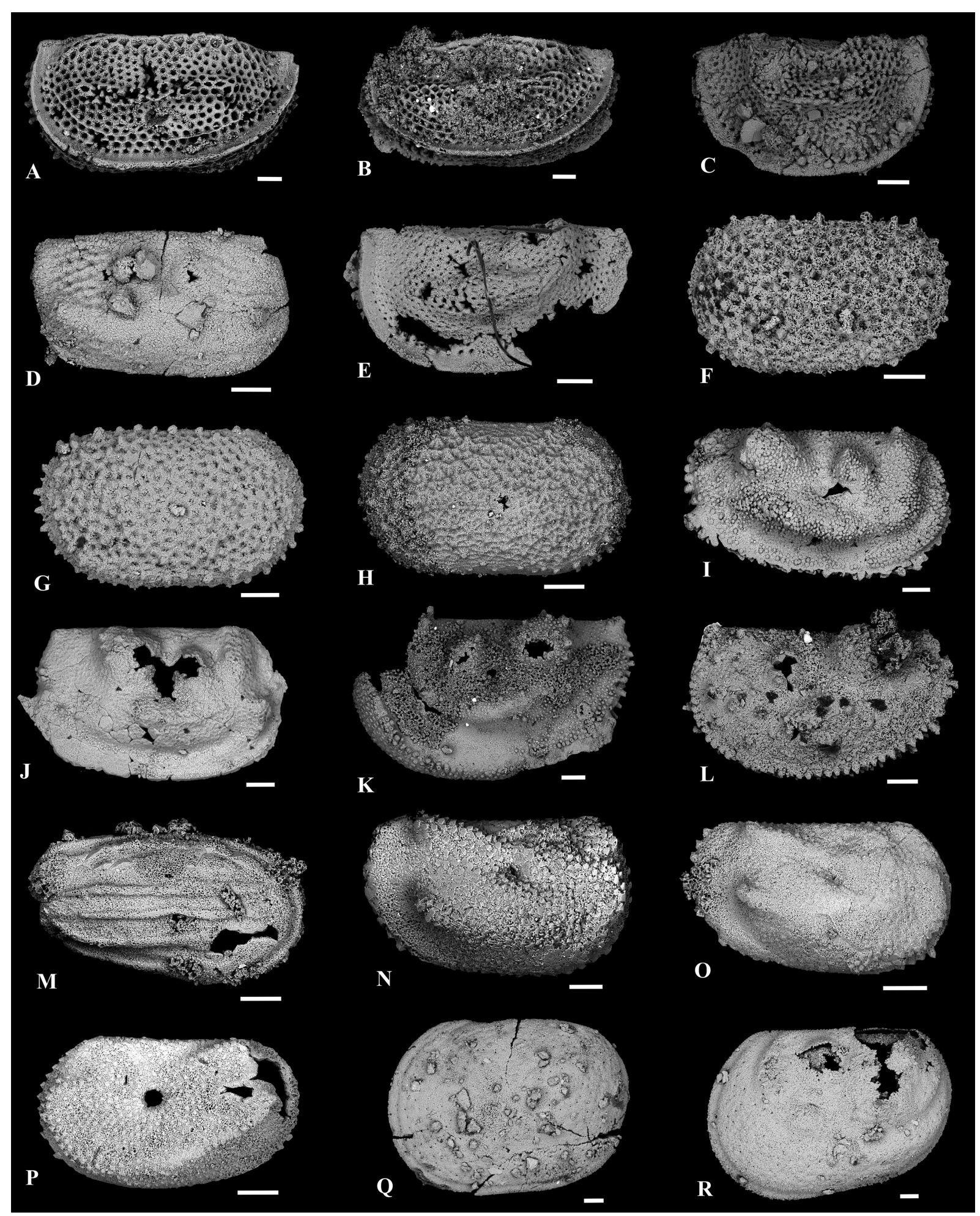


Paratype

USA 1 complete carapace, (Fig. 6B); Guadalupe Mountains, West Texas, Williams Ranch Member, Cutoff Formation, Quarry section, sample GM1; Roadian, Middle Permian; MNHN.F.F63384.

\section{Additional material}

USA $\bullet 8$ valves; Guadalupe Mountains, West Texas, Williams Ranch Member, Cutoff Formation, Quarry section, samples GM1, GM2, and GM3; Roadian, Middle Permian; MNHN.F.F63478.

\section{Dimensions}

$\mathrm{L}=829-1280 \mu \mathrm{m} ; \mathrm{H}=368-660 \mu \mathrm{m}$ (Fig. 5B).

\section{Description}

Carapace sub-rectangular in lateral view with Hmax located in anterior half; Lmax located in dorsal part of Hmax; DB long and straight; posterior shoulder clearly expressed, ACA close to $90^{\circ}$ or slightly obtuse; PCA close to $90^{\circ}$ or slightly acute; $\mathrm{AB}$ with large radius of curvature with maximum located around mid-H; VB almost straight with weak median concavity, parallel to DB; PB with small radius of curvature and maximum located close to DB; 2 vertical lobes connected above kirkbyan pit by small sub-horizontal lobe; kirkbyan pit located at mid-L and below mid-H; two important ridges along free margins, one ventral and one adventral; marginal structure underlined by tubercles; adventral ridge smooth as well as areas around cardinal angles; additional ridge parallel to adventral one, extending from mid-H in anterior part up to mid- $\mathrm{H}$ in posterior part of the carapace; presence of a dorsal shield characteristic of the genus; surface reticulate.

\section{Occurrence}

Samples GM1, GM2, and GM3, Quarry section, Williams Ranch Member, Cutoff Formation, Guadalupe Mountains, West Texas, USA, Roadian, Middle Permian (this work).

\section{Remarks}

Aurikirkbya guadalupensis sp. nov. differs from the three species described by Sohn (1950) (A. wordensis (Hamilton, 1942), A. barbarae Sohn, 1950 and A. auriformis Sohn, 1950) from the Roadian (upper Leonardian or lower Wordian in the sense of Sohn 1950) of the Glass Mountains, Texas by a smaller PB and a diagnostic ridge. Aurikirkbya kelletae (Harlton, 1929) from the Pennsylvanian of Texas and Aurikirkbya miyakei Yanaka \& Ono in Tanaka et al., 2012 from the Early Permian of Japan (Tanaka et al. 2012) have a similar carapace shape but differ by the diagnostic ridge.

Aurikirkbya wordensis (Hamilton, 1942)

Fig. 6C, E

Kirkbya wordensis Hamilton, 1942: 713-714, pl. 110 fig. 13.

Aurikirkbya wordensis - Sohn 1950: pl. 7, figs 1-13.

\section{Material examined}

USA • 1 left valve; Guadalupe Mountains, West Texas, 'McKittrick Canyon' Member, Bell Canyon Formation, MKCS1 section, sample 2 (GM6); Capitanian, Middle Permian; MNHN.F.F63385 • 1 valve; Guadalupe Mountains, West Texas, Williams Ranch Member, Cutoff Formation, Quarry section, sample GM4; Roadian; MNHN.F.F63387 - 1 valve; same locality as for preceding; 'McKittrick Canyon' Member, Bell Canyon Formation, MKCS1 section, sample 4 (GM5); Capitanian, Middle Permian; MNHN.F.F63479. 


\section{Occurrence}

Texas, USA, Roadian, Middle Permian (Hamilton 1942; Sohn 1950); sample GM4, Quarry section, Williams Ranch Member, Cutoff Formation, Roadian; samples GM5 and GM6, MKCS1 section, 'McKittrick Canyon' Member, Bell Canyon Formation, Guadalupe Mountains, West Texas, USA, Capitanian, Middle Permian (this work).

Family Kirkbyellidae Sohn, 1961

Genus Kirkbyella Coryell \& Booth, 1933

\section{Type species}

Kirkbyella typa Coryell \& Booth, 1933 by original designation.

Kirkbyella (Berdanella) cf. quadrata Croneis \& Gutke, 1939

Fig. 6D

\section{Material examined}

USA 11 right valve; Guadalupe Mountains, West Texas, 'McKittrick Canyon' Member, Bell Canyon Formation, MKCS1 section, sample 4 (GM5); Capitanian, Middle Permian; MNHN.F.F63386.

\section{Remarks}

This specimen could belong to a new species with regard to the ornamentation of the carapace that consists of small ridges on the anterior part and a reticulation on the posterior part. The general shape is similar to that of Kirkbyella (Berdanella) quadrata Croneis \& Gutke, 1939 sensu Hoare \& Mapes 2000 from the Mississippian of Arkansas (Hoare \& Mapes 2000) but in this species the reticulation is organised quite horizontally and here it is more oblique.

\section{Occurrence}

Sample 4 (GM5), MKCS1 section, 'McKittrick Canyon' Member, Bell Canyon Formation, Guadalupe Mountains, West Texas, USA, Capitanian, Middle Permian (this work).

Family Scrobiculidae Posner, 1951

Genus Roundyella Bradfield, 1935

\section{Type species}

Amphissites simplicissimus Knight, 1928 subsequently designated by Bradfield (1935).

Roundyella lebaensis Krömmelbein, 1958

Fig. $6 \mathrm{~F}-\mathrm{H}$

Roundyella lebaensis Krömmelbein, 1958: 118, pl. 1 figs 11-15, pl. 3 figs 45-46.

Roundyella krommelbeini sp. nov. Kozur, 1991: 15, 16, pl. 7, fig. 2.

Roundyella lebaensis wangi n. subsp. Kozur, 1991: 15, 16, pl. 8, figs 2, 13.

Roundyella lebaensis - Knüpfer 1967: 76, pl. 1 fig. 1. - Ivanov 1975: 164, pl. 33 fig. 34, pl. 39 fig. 4. — Woszczyńska 1987: 188, pl. 9 figs 1-2, 8. — Olempska \& Błaszyk 1996: 15, pl. 2 fig. 5, pl. 4 fig. 1. 


\section{Material examined}

USA - 1 left valve; Guadalupe Mountains, West Texas, Williams Ranch Member, Cutoff Formation, Quarry section, samples GM4; Roadian, Middle Permian; MNHN.F.F63388 • 1 right valve; same locality as for preceding; Roadian, Middle Permian; MNHN.F.F63389 • 1 right valve; Guadalupe Mountains, West Texas, Williams Ranch Member, Cutoff Formation, Quarry section, samples GM1; Roadian, Middle Permian; MNHN.F.F63390 • 5 valves; Guadalupe Mountains, West Texas, Williams Ranch Member, Cutoff Formation, Quarry section, samples GM1, GM4; Roadian; MNHN.F.F63654 • 3 valves; same locality as for preceding; 'McKittrick Canyon' Member, Bell Canyon Formation, MKCS1 section, sample 4 (GM5) Capitanian; Middle Permian; MNHN.F.F63480.

\section{Occurrence}

German basin, Werrakarbonate, Zechstein (Knüpfer 1967); USSR, Baltic region, Novoakmyanskaya svita (Ivanov 1975); Polish lowlands, cyclothem PZ1, Zechstein (Woszczyńska 1987); Bükk Mountain, Hungary, Wuchiapingian, Late Permian (Kozur 1991); southwest Spitzbergen, Norway, Wuchiapingian, Late Permian (Olempska \& Błaszyk 1996); samples GM1, GM4, Quarry section, Williams Ranch Member, Cutoff Formation, Roadian; sample 4 (GM5), MKCS1 section, 'McKittrick Canyon' Member, Bell Canyon Formation, Capitanian, Guadalupe Mountains, West Texas, USA, Middle Permian (this work).

Superfamily Hollinoidea Swartz, 1936

Family Hollinellidae Bless \& Jordan, 1971

Genus Hollinella Coryell, 1928

\section{Type species}

Hollinella dentata Coryell, 1928 by original designation.

Hollinella (Hollinella) williamsranchensis Crasquin sp. nov. urn:lsid:zoobank.org:act:2A663FBA-1BBF-481E-B29E-1EF99A05F293

Figs $5 \mathrm{C}, 6 \mathrm{I}-\mathrm{L}$

\section{Diagnosis}

A species of Hollinella (Hollinella) with a subvertical $\mathrm{L}_{1}$ in continuation of the ventral lobe and dorsally ended by a small nodule/spine; spine at the posterior part of the velum.

\section{Etymology}

After Williams Ranch Member, Bell Canyon Formation, Capitanian, West Texas where this species has been discovered.

\section{Material examined}

\section{Holotype}

USA - right valve, (Fig. 6I); Guadalupe Mountains, West Texas, Williams Ranch Member, Cutoff Formation, Quarry section, sample GM1; Roadian, Middle Permian; MNHN.F.F63391.

\section{Paratype}

USA • 1 left valve, (Fig. 6K); Guadalupe Mountains, West Texas, Williams Ranch Member, Cutoff Formation, Quarry section, sample GM1; Roadian, Middle Permian; MNHN.F.F63393. 
Additional material

USA 1 right valve; Guadalupe Mountains, West Texas, Williams Ranch Member, Cutoff Formation, Quarry section, sample GM1; Roadian, Middle Permian; MNHN.F.F63392 • 1 left valve; same locality as for preceding but sample GM4; Roadian, Middle Permian; MNHN.F.F63394 • 2 valves; Guadalupe Mountains, West Texas, Williams Ranch Member, Cutoff Formation, Quarry section, samples GM1, GM4, Roadian; MNHN.F.F63655 • 6 valves; same locality as for preceding; 'McKittrick Canyon' Member, Bell Canyon Formation, MKCS1 section, sample 4 (GM5), Capitanian; Middle Permian; MNHN.F.F63481.

\section{Dimensions}

$\mathrm{L}=776-1318 \mu \mathrm{m} ; \mathrm{H}=434-773 \mu \mathrm{m}$ (Fig. 5C).

\section{Description}

Carapace auriform and elongate in lateral view; Hmax at ACA; Lmax at mid-L; DB long and straight; presence of cardinal spine at PCA; AB with maximum of curvature at or below mid-H; VB straight; $\mathrm{PB}$ with quite large radius of curvature underlined by nodules; presence of a granular velum that ends by posterior spine; $\mathrm{L}_{1}$ well expressed, vertical and in continuation of ventral lobe, ended dorsally by small nodule/spine; $\mathrm{L}_{2}$ well expressed, peaked, with small nodule; $\mathrm{L}_{3}$ covered by nodules, higher than $\mathrm{DB}$; surface granular; intraspecific variations observed in velum formed by small spines (Fig. 6L) and blade (Fig. 6I).

\section{Occurrence}

Samples GM1, GM4, Quarry section, Williams Ranch Member, Cutoff Formation, Roadian; sample 4(GM5) MKCS1 section, 'McKittrick Canyon' Member, Bell Canyon Formation, Capitanian; Guadalupe Mountains, West Texas, USA, Middle Permian (this work).

\section{Remarks}

Hollinella (Hollinella) williamsranchensis sp. nov. is reminiscent of $H$. warthini Cooper, 1946 from the Missourian-Virgilian (Late Pennsylvanian) of USA (see synthesis in Christopher et al. 1990) by the carapace shape, spines at PCA and posterior part of the velum and the granular surface. However, in H. warthini, $\mathrm{L}_{1}$ is not visible and $\mathrm{L}_{2}$ and $\mathrm{L}_{3}$ are more bulbous. Hollinella (Hollinella) williamsranchensis sp. nov. has the same ornamentation on $\mathrm{L}_{3}$ as $H$. (H.) lungcamensis Crasquin, 2018 from the PermianTriassic boundary of North Vietnam (Crasquin et al. 2018), which is stockier $(\mathrm{H} / \mathrm{L}=0.55-0.65$ herein, $0.65-0.75$ for $H$. (H.) lungamensis).

Superfamily Kloedenelloidea Ulrich \& Bassler, 1908

Family Glyptopleuridae Girty, 1910

Genus Glyptopleura Girty, 1910

\section{Type species}

Glyptopleura inopinata Girty, 1910 by original designation.

\section{Glyptopleura sp.}

Fig. 6M

\section{Material examined}

USA 1 right valve; Guadalupe Mountains, West Texas, Williams Ranch Member, Cutoff Formation, Quarry section, sample GM1; Roadian, Middle Permian; MNHN.F.F63395 • 7 valves; Guadalupe Mountains, West Texas, Williams Ranch Member, Cutoff Formation, Quarry section, samples GM1, GM2, and GM3; Roadian, Middle Permian; MNHN.F.F63482. 


\section{Remarks}

A species of Glyptopleura with 6 thick ridges on the lateral surface, the upper two ridges are shorter than the others and end before PB. This species may be new, but the material is poorly preserved. So far, this species is the youngest representative of Glyptopleura.

\section{Occurrence}

Samples GM1, GM2, and GM3, Quarry section, Williams Ranch Member, Cutoff Formation, Guadalupe Mountains, West Texas, USA, Roadian, Middle Permian (this work).

Family Geisinidae Sohn, 1961b

Genus Geisina Johnson, 1936

\section{Type species}

Beyrichiella gregaria Ulrich \& Bassler, 1906 subsequently designated by Johnson (1936).

Geisina culbersonensis Crasquin sp. nov. urn:1sid:zoobank.org:act:FA68573E-E0FA-4DC1-B82E-6F860628863F

Fig. $6 \mathrm{~N}-\mathrm{P}$

\section{Diagnosis}

A species of Geisina with a pustulose carapace, two large spines in the posterior part of the carapace and free margins bordered with small spines.

\section{Etymology}

From Culberson County, West Texas, USA, where the studied samples have been collected.

\section{Material examined}

Holotype

USA - complete carapace, (Fig. 6N); Guadalupe Mountains, West Texas, Williams Ranch Member, Cutoff Formation, Quarry section, sample GM1; Roadian, Middle Permian; MNHN.F.F63396.

\section{Paratype}

USA • 1 complete carapace, (Fig. 6O); Guadalupe Mountains, West Texas, Williams Ranch Member, Cutoff Formation, Quarry section, sample GM1; Roadian, Middle Permian; MNHN.F.F63397.

\section{Additional material}

USA $\cdot 1$ complete carapace; Guadalupe Mountains, West Texas, Williams Ranch Member, Cutoff Formation, Quarry section, sample GM2; Roadian, Middle Permian; MNHN.F.F63398 1 complete carapace, 4 valves; Guadalupe Mountains, West Texas, Williams Ranch Member, Cutoff Formation, Quarry section, samples GM1, GM2, GM4; Roadian, Middle Permian; MNHN.F.F63483.

\section{Dimensions}

$\mathrm{L}=602-676 \mu \mathrm{m} ; \mathrm{H}=430-548 \mu \mathrm{m} ; \mathrm{H} / \mathrm{L}=0.53-0.64$.

\section{Description}

Carapace sub-rectangular in lateral view with Hmax in anterior $1 / 3^{\mathrm{rd}}$ of $\mathrm{L}$ and Lmax slightly below mid-H; DB long and straight; $\mathrm{ACA}=115^{\circ}-130^{\circ}, \mathrm{PCA}=105^{\circ}-115^{\circ}$; $\mathrm{AB}$ with large radius of curvature with maximum at lower $1 / 3^{\text {rd }} \mathrm{H}$; VB nearly straight; PB laterally compressed, large with maximum of curvature located at mid- $\mathrm{H}$; free margins bordered by small spines; $\mathrm{L}_{2}$ not well visible; $\mathrm{S}_{2}$ round and 
clearly marked; $\mathrm{L}_{3}$ underlined by two large spines, one dorsal and one medio-ventral, pointing upward; surface completely covered with pustules.

\section{Occurrence}

Samples GM1, GM2, GM4, Quarry section, Williams Ranch Member, Cutoff Formation, Guadalupe Mountains, Western Texas, USA, Roadian, Middle Permian (this work).

\section{Remarks}

Geisina culbersonensis sp. nov. has a very particular shape and primary ornamentation. It can be compared with Geisina sp. in Chitnarin et al. 2012 from the Asselian-Sakmarian, Early Permian of Thailand (Chitnarin et al. 2012), which has a smaller PB and a smooth surface.

Family Miltonellidae Sohn, 1950

Genus Miltonella Sohn, 1950

\section{Type species}

Miltonella shupei Sohn, 1950 by original designation.

Miltonella ef. shupei Sohn, 1950

Fig. 6Q-R

\section{Material examined}

USA - 1 left valve; Guadalupe Mountains, West Texas, 'McKittrick Canyon' Member, Bell Canyon Formation, MKCS1 section, sample 4 (GM5); Capitanian, Middle Permian; MNHN.F.F63399 • 1 left valve; same locality as for preceding; Capitanian, Middle Permian; MNHN.F.F63400 2 right valves; same locality as for preceding; Capitanian, Middle Permian; MNHN.F.F63484.

\section{Occurrence}

Sample 4 (GM5), MKCS1 section, 'McKittrick Canyon' Member, Bell Canyon Formation, Guadalupe Mountains, West Texas, USA, Capitanian, Middle Permian (this work).

\section{Remarks}

This species is similar to Miltonella shupei Sohn, 1950 from the Middle Permian of Texas (Sohn 1950). Herein, the specimens are shorter and the characteristic ridge of the genus begins at ACA and finishes at PCA (instead of PB in type species).

$$
\text { ?Family Miltonellidae Sohn, } 1950
$$

Genus Libumella Rozhdestvenskaya, 1959

\section{Type species}

Libumella discoides Rozhdestvenskaya, 1959 by original designation.

\section{Libumella cf. athabascensis Green, 1963}

Fig. 7A

\section{Material examined}

USA - 1 complete carapace; Guadalupe Mountains, West Texas, Williams Ranch Member, Cutoff Formation, Quarry section, sample GM1; Roadian, Middle Permian; MNHN.F.F63401. 


\section{Occurrence}

Sample GM1, Quarry section, Williams Ranch Member, Cutoff Formation, Guadalupe Mountains, West Texas, USA, Roadian, Middle Permian (this work).

\section{Remarks}

The specimen is similar to Libumella athabascensis Green, 1963 from the Mississippian of the Rocky Mountains, Canada (Green 1963; Crasquin 1985) and from the Late Permian of South China (Yuan et al. 2007). Nevertheless, it differs by the presence of a nodule in the dorso-median part of the carapace.

Order Leiocopa Schallreuter, 1973

Superfamily Paraparchitoidea Scott, 1959

Family Paraparchitidae Scott, 1959

Genus Paraparchites Ulrich \& Bassler, 1906

\section{Type species}

Paraparchites humerosus Ulrich \& Bassler, 1906 by original designation.

Paraparchites texanus Delo, 1930

Fig. 7C

Paraparchites humerosus var. texana Delo, 1930: 153, pl. 12 fig. 1.

Paraparchites texanus - Sohn 1971: A8, pl. 2 figs 1-2, 7-10, 13-15, with all previous synonyms therein. non Paraparchites texanus - Crasquin-Soleau \& Orchard 1994: 59, 60, pl. 4 fig. 12. — Yi 2004: pl. 2 fig. 13.

Fig. 7 (next page). SEM micrographs of ostracods from the Middle Permian (Roadian, Quarry section, samples GM1 and GM2; Capitanian, MKCS1 section, samples 4 (GM5) and 2 (GM6)) of the Guadalupe Mountains (West Texas, USA). All specimens are temporarily housed in the collections of the Muséum national d'histoire naturelle, Paris, France (MNHN). A. Libumella cf. athabascensis Green, 1963, left lateral view of a carapace, sample GM1 (MNHN.F.F63401). - B. Fellerites? sp., external view of a right valve, sample GM1 (MNHN.F.F63402). - C. Paraparchites texanus Delo, 1930, right lateral view of a carapace, sample 4 (GM5) (MNHN.F.F63403). - D-H. Paraparchites pecosensis Crasquin sp. nov. D. Holotype, right lateral view of a carapace, sample GM1 (MNHN.F.F63404). E. Right lateral view of a carapace, sample GM1 (MNHN.F.F63405). F. External view of a right valve, sample 2 (GM6) (MNHN.F.F63406). G. External view of a right valve, sample GM1 (MNHN.F.F63407). H. Paratype, left lateral view of a carapace, sample GM1 (MNHN.F.F63408). - I. Acratia sp., right lateral view of a carapace, sample GM2 (MNHN.F.F63409). - J. Acratia cf. sinuata (Kozur, 1991), external view of a left valve, sample GM1 (MNHN.F.F63410). - K. Acratia sinuata (Kozur, 1991), external view of a left valve, sample GM1 (MNHN.F.F63411). - L-M. Bairdia radlerae Kellett, 1934. L. External view of a left valve, sample GM1 (MNHN.F.F63412). M. Right lateral view of a carapace, sample GM1 (MNHN.F.F63413). - N. Bairdia rhomboidalis Hamilton, 1942, right lateral view of a carapace, sample 2 (GM6) (MNHN.F.F63414). - O. Bairdia subfusiformis Hamilton, 1942, external view of a left valve, sample GM1 (MNHN.F.F63415). - P-R. Bairdia elcapitanensis Forel sp. nov. P. Right lateral view of a carapace, sample GM1 (MNHN.F.F63416). Q. Right lateral view of a carapace, sample GM1 (MNHN.F.F63417). R. Paratype, external view of a right valve, sample GM1 (MNHN.F.F63418). Scale bars: $100 \mu \mathrm{m}$. 


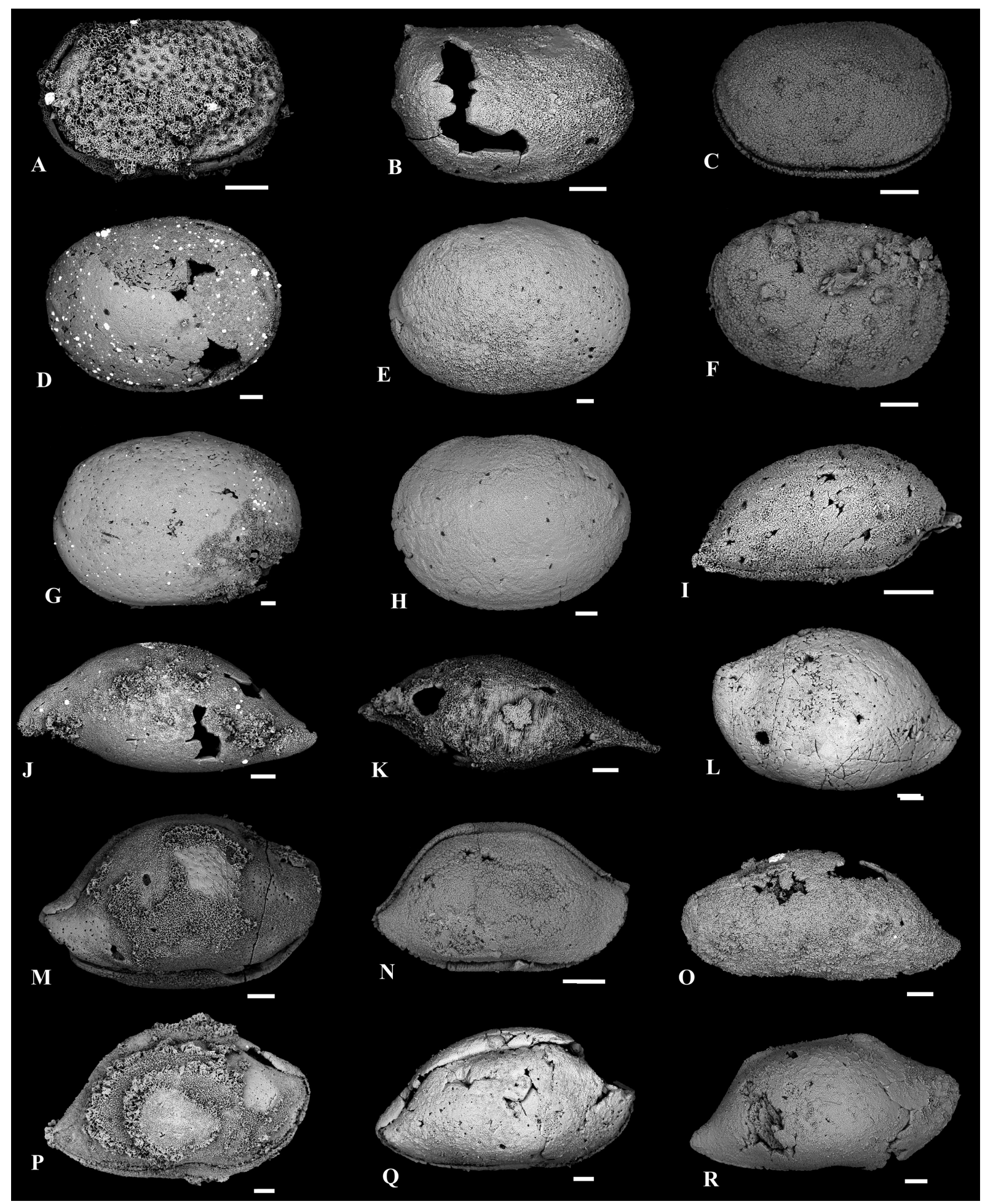




\section{Material examined}

USA 11 complete carapace; Guadalupe Mountains, West Texas, 'McKittrick Canyon' Member, Bell Canyon Formation, MKCS1 section, sample 4 (GM5); Capitanian, Middle Permian; MNHN.F.F63403

- 2 complete carapaces; same locality as for preceding; Capitanian, Middle Permian; MNHN.F.F63485.

\section{Occurrence}

Menar and Tom Green Counties, Texas, USA, Kungurian (Arroyo Formation), Early Permian (Sohn 1971 and all references therein); sample 4 (GM5), MKCS1 section, 'McKittrick Canyon' Member, Bell Canyon Formation, Capitanian; Guadalupe Mountains, West Texas, USA, Guadalupian, Middle Permian (this work).

\section{Paraparchites pecosensis Crasquin sp. nov. urn:lsid:zoobank.org:act:E76693E7-FD8A-4E7B-9328-6D3248AC584F}

Figs 5D, 7D-H

\section{Diagnosis}

A species of Paraparchites with a small hemi-circular ridge at mid-H of PB on both valves and a weak overreach of RV above hinge-line.

\section{Etymology}

In reference to the Pecos River, West Texas, USA.

\section{Material examined}

\section{Holotype}

USA - complete carapace, (Fig. 7D); Guadalupe Mountains, West Texas, Williams Ranch Member, Cutoff Formation, Quarry section, sample GM1; Roadian, Middle Permian; MNHN.F.F63404.

\section{Paratype}

USA - 1 complete carapace, (Fig. 7H); Guadalupe Mountains, West Texas, Williams Ranch Member, Cutoff Formation, Quarry section, sample GM1; Roadian, Middle Permian; MNHN.F.F63408.

\section{Additional material}

USA - 1 complete carapace; Guadalupe Mountains, West Texas, Williams Ranch Member, Cutoff Formation, Quarry section, sample GM1; Roadian, Middle Permian; MNHN.F.F63405 - 1 right valve; same locality as for preceding; Roadian, Middle Permian; MNHN.F.F63407 • 1 right valve; Guadalupe Mountains, West Texas, 'McKittrick Canyon' Member, Bell Canyon Formation, MKCS1 section, sample GM6; Capitanian, Middle Permian; MNHN.F.F63406 • 1 complete carapace, 52 valves; Guadalupe Mountains, West Texas, Williams Ranch Member, Cutoff Formation, Quarry section, samples GM1, GM2, GM3; Roadian; MNHN.F.F63656 • 15 valves; same locality as for preceding; 'McKittrick Canyon' Member, Bell Canyon Formation, MKCS1 section, sample 2 (GM6), Capitanian; Middle Permian; MNHN.F.F63486.

\section{Dimensions}

$\mathrm{L}=503-2100 \mu \mathrm{m} ; \mathrm{H}=341-1467 \mu \mathrm{m}$ (Fig. 5D).

\section{Description}

Carapace stocky $(\mathrm{H} / \mathrm{L}=0.69-0.75)$ sub-rectangular in lateral outline; $\mathrm{DB}$ straight at $\mathrm{LV}$; DB a little convex with weak overreach of RV above hinge-line; $\mathrm{AB}$ and $\mathrm{PB}$ quite equivalent with large radius of curvature, maximum of curvature around mid-H; VB nearly straight; LV overlaps RV all around free margins; presence of small hemi-circular ridge at mid-H of PB on both valves. 


\section{Occurrence}

Samples GM1, GM2, and GM3, Quarry section, Williams Ranch Member, Cutoff Formation, Roadian; sample 2 (GM6), MKCS1 section, 'McKittrick Canyon' Member, Bell Canyon Formation, Capitanian; Guadalupe Mountains, West Texas, USA, Middle Permian (this work).

\section{Remarks}

Paraparchites pecosensis sp. nov. has a lateral outline similar to that of Paraparchites texanus Ulrich \& Bassler, 1906 emend. Scott, 1959 from the Early Permian of Texas (Scott 1959). The main difference between the two species is the small hemi-circular ridge at PB in the new species.

Order Podocopida Sars, 1866

Suborder Podocopina Sars, 1866

Superfamily Bairdioidea Sars, 1888

Family Bairdiidae Sars, 1888

\section{Preliminary remarks}

The classification of ornate Bairdiidae is complex and still disputed mainly because of the difficulty to unravel the taxonomic significance of their lateral ornamentation features. As detailed in Forel \& Grădinaru (2020), the revision of their classification requires the addition of new characters to avoid producing another classification system that would only be a new point of view further complicating the current situation. The peak of diversity of ornate Bairdiidae occurred in the Late Triassic but they were already present in the Permian (see Forel \& Crasquin 2020 and references therein for a review) as illustrated for instance by the material described herein from the Middle Permian of the Guadalupe Mountains. Specifically, the confusion and instability of the conceptions of Petasobairdia Chen, 1982 and Praelobobairdia Kozur, 1991 have recently been described and discussed in Forel \& Grădinaru (2020). Herein, we follow the view that Praelobobairdia is a junior synonym of Petasobairdia, as was first introduced by Becker (2001).

Genus Acratia Delo, 1930

\section{Type species}

Acratia typica Delo, 1930 by original designation.

Acratia sinuata (Kozur, 1991)

Fig. $7 \mathrm{~K}$

Cooperuna sinuata Kozur, 1991: 107-108, pl. 21 fig. 2.

\section{Material examined}

USA 1 left valve; Guadalupe Mountains, West Texas, Williams Ranch Member, Cutoff Formation, Quarry section, sample GM1; Roadian, Middle Permian; MNHN.F.F63411 • 1 complete carapace, 2 valves; Guadalupe Mountains, West Texas, Williams Ranch Member, Cutoff Formation, Quarry section, samples GM1, GM4; Roadian, Middle Permian; MNHN.F.F63487.

\section{Occurrence}

Bükk Mountains, Hungary, Early Wuchiapingian, Late Permian (Kozur 1991); samples GM1, GM4, Quarry section, Williams Ranch Member, Cutoff Formation, Guadalupe Mountains, West Texas, USA, Roadian, Middle Permian (this work). 
Genus Bairdia McCoy, 1844

\section{Type species}

Bairdia curta McCoy, 1844 subsequently designated by Ulrich \& Bassler (1923).

\section{Bairdia radlerae Kellett, 1934}

Fig. 7L-M

Bairdia radlerae Kellett, 1934: 125, pl. 14 figs 3a-c.

Bairdia radlerae - Sohn 1960: 31.

\section{Material examined}

USA - 1 left valve; Guadalupe Mountains, West Texas, Williams Ranch Member, Cutoff Formation, Quarry section, sample GM1; Roadian, Middle Permian; MNHN.F.F63412 • 1 complete carapace; same locality as for preceding; Roadian, Middle Permian; MNHN.F.F63413 • 78 valves; Guadalupe Mountains, West Texas, Williams Ranch Member, Cutoff Formation, Quarry section, samples GM1, GM2, and GM4; Roadian; MNHN.F.F63657 • 27 valves; same locality as for preceding; 'McKittrick Canyon' Member, Bell Canyon Formation, MKCS1 section, samples 4 (GM5) and 2 (GM6), Capitanian; Middle Permian; MNHN.F.F63488.

\section{Occurrence}

Kansas, USA, Asselian, Sakmarian, Early Permian (Kellett 1934); samples GM1, GM2, GM4, Quarry section, Williams Ranch Member, Cutoff Formation, Roadian; samples 4 (GM5) and 2 (GM6), MKCS1 section, 'McKittrick Canyon' Member, Bell Canyon Formation, Capitanian; Middle Permian, Guadalupe Mountains, West Texas, USA (this work).

Bairdia rhomboidalis Hamilton, 1942

Fig. $7 \mathrm{~N}$

Bairdia rhomboidalis Hamilton, 1942: 715, pl. 110 figs 11a-b.

Bairdia rhomboidalis - Sohn 1960: 31, pl. 1 figs 26-27.

\section{Material examined}

USA 11 complete carapace; Guadalupe Mountains, West Texas, 'McKittrick Canyon' Member, Bell Canyon Formation, MKCS1 section, sample 2 (GM6); Capitanian, Middle Permian; MNHN.F.F63414 • 1 complete carapace; same locality as for preceding but sample 4 (GM5); Capitanian, Middle Permian; MNHN.F.F63489.

\section{Occurrence}

Texas, USA, Roadian, Middle Permian (Hamilton 1942); samples 4 (GM5) and 2 (GM6), MKCS1 section, 'McKittrick Canyon' Member, Bell Canyon Formation, Guadalupe Mountains, West Texas, USA, Capitanian, Middle Permian (this work).

\section{Bairdia subfusiformis Hamilton, 1942}

Fig. 70

Bairdia subfusiformis Hamilton, 1942: pl. 110, figs 8a-b.

Bairdia subfusiformis - Sohn 1960: 32. 


\section{Material examined}

USA 11 left valve; Guadalupe Mountains, West Texas, Williams Ranch Member, Cutoff Formation, Quarry section, sample GM1; Roadian, Middle Permian; MNHN.F.F63415 • 1 left valve; Guadalupe Mountains, West Texas, Williams Ranch Member, Cutoff Formation, Quarry section, sample GM1, Roadian; MNHN.F.F63658 • 1 left valve; same locality as for preceding; 'McKittrick Canyon' Member, Bell Canyon Formation, sample 4 (GM5); Capitanian, Middle Permian; MNHN.F.F63490.

\section{Occurrence}

Glass Mountains, West Texas, USA, Roadian, Middle Permian (Hamilton 1942); sample GM1, Quarry section, Williams Ranch Member, Cutoff Formation, Roadian; sample 4 (GM5), MKCS1 section, 'McKittrick Canyon' Member, Bell Canyon Formation, Capitanian; Guadalupe Mountains, West Texas, USA, Middle Permian (this work).

Bairdia elcapitanensis Forel sp. nov. urn:1sid:zoobank.org:act:4DDDDD5C-6D2B-45E5-8B59-427C19C96DD7

Figs $7 \mathrm{P}-\mathrm{R}, 8 \mathrm{~A}-\mathrm{B}$

\section{Diagnosis}

A species of Bairdia with asymmetric valves and with a strong overlap along DB; ADB concave at RV and dorsal margin uniformly convex; $\mathrm{PD}$ and $\mathrm{AD}$ angles laterally compressed at $\mathrm{RV}$.

\section{Etymology}

In reference to the El Capitan Peak located within the Guadalupe Mountains National Park, Guadalupe Mountains, West Texas, USA.

\section{Material examined}

\section{Holotype}

USA • right valve, (Fig. 8B); Guadalupe Mountains, West Texas, Williams Ranch Member, Cutoff Formation, Quarry section, sample GM1; Roadian, Middle Permian; MNHN.F.F63420.

\section{Paratype}

USA • 1 right valve, (Fig. 7R); Guadalupe Mountains, West Texas, Williams Ranch Member, Cutoff Formation, Quarry section, sample GM1; Roadian, Middle Permian; MNHN.F.F63418.

\section{Additional material}

USA -1 complete carapace; Guadalupe Mountains, West Texas, Williams Ranch Member, Cutoff Formation, Quarry section, sample GM1; Roadian, Middle Permian; MNHN.F.F63416 • 1 carapace; same locality as for preceding; Roadian, Middle Permian; MNHN.F.F63417 • 1 right valve; same locality as for preceding; Roadian, Middle Permian; MNHN.F.F634119 • 16 right valves; same locality as for preceding but samples GM1, GM2; Roadian, Middle Permian; MNHN.F.F63491.

\section{Dimensions}

$\mathrm{L}=1250-1386 \mu \mathrm{m} ; \mathrm{H}=635-761 \mu \mathrm{m}$.

\section{Description}

Carapace sub-triangular in lateral view with asymmetric valves and overlap of LV all around RV with maximum along DB; lateral surface smooth. 
LV: Hmax around mid-L; dorsal margin uniformly convex with PDB and ADB almost straight; AB with small radius of curvature located above mid-H; VB straight to only gently concave; PB narrow with maximum of convexity located very ventrally.

RV: Hmax located at AD angulation; DB long and straight, gently bent posteriorly; PDB straight with terminal concavity; $\mathrm{ADB}$ concave; $\mathrm{PD}$ angle $\left(130-135^{\circ}\right)$ and $\mathrm{AD}$ angle $\left(135-140^{\circ}\right)$ laterally compressed; $\mathrm{AB}$ with relatively small radius of curvature, located above mid-H; oral concavity in front of mid-L at ventral margin; PB slighted.

\section{Occurrence}

Samples GM1 and GM2, Quarry section, Williams Ranch Member, Cutoff Formation, Guadalupe Mountains, West Texas, USA, Roadian, Middle Permian (this work).

\section{Remarks}

Bairdia girtyi Sohn, 1960 sensu Hoare \& Merrill, 2004 from the Pennsylvanian of Texas (Hoare \& Merrill 2004) is similar to Bairdia elcapitanensis sp. nov. by its lateral morphology, but lacks the AD and PD angulations at RV. It is worth noting that the specimens of Hoare \& Merrill (2004) do not belong to Bairdia girtyi Sohn, 1960 from the Late Mississippian of USA (Sohn 1960), because Bairdia girtyi is more elongate, with PB located higher and Hmax located slightly behind mid-H.

\section{Bairdia sp. A}

Fig. 8D

\section{Material examined}

USA 1 complete carapace; Guadalupe Mountains, Williams Ranch Member, Cutoff Formation, Quarry section, sample GM1; Roadian, Middle Permian; MNHN.F.F63422 • 1 complete carapace, 4 valves; same locality as for preceding; Roadian, Guadalupian, Middle Permian; MNHN.F.F63492.

Fig. 8 (next page). SEM micrographs of ostracods from the Middle Permian (Roadian, Quarry section, samples GM1 and GM4; Capitanian, MKCS1 section, samples 4 (GM5) and 2 (GM6)) of the Guadalupe Mountains (West Texas, USA). All specimens are temporarily housed in the collections of the Muséum national d'histoire naturelle, Paris, France (MNHN). A-B. Bairdia elcapitanensis Forel sp. nov. A. External view of a right valve, sample GM1 (MNHN.F.F63419). B. Holotype, external view of a right valve, sample GM1 (MNHN.F.F63420). - C. Bairdia cf. postacuta Chen, 1958, right lateral view of a carapace, sample GM1 (MNHN.F.F63421). - D. Bairdia sp. A, right lateral view of a carapace, sample GM1 (MNHN.F.F63422). - E. Bairdia sp. B, right lateral view of a carapace, sample GM1 (MNHN.F.F63423). - F. Bairdia sp. C, right lateral view of a carapace, sample GM1 (MNHN.F.F63424) - G. Bairdiacypris sp. B, right lateral view of a carapace, sample 2 (GM6) (MNHN.F.F63425). - H. Bairdiacypris sp. C, right lateral view of a carapace, sample GM1 (MNHN.F.F63426). I-J. Fabalicypris acetalata (Coryell \& Billings, 1932). I. Right lateral view of a carapace, sample GM1 (MNHN.F.F63427). J. External right of a right valve, sample GM4 (MNHN.F.F63428). K. Fabalicypris glennensis (Harlton, 1927), right lateral view of a carapace, sample 4 (GM5) (MNHN.F.F63429). - L. Fabalicypris sp., right lateral view of a carapace, sample GM4 (MNHN.F.F63430). - M-N. Orthobairdia texana (Harlton, 1927). M. External right of a right valve, sample 4 (GM5) (MNHN.F.F63431). N. External view of a right valve, sample GM1 (MNHN.F.F63432). - O-R. Ceratobairdia mescaleroella Forel sp. nov. O. Holotype, adult, external view of a left valve, sample 4 (GM5) (MNHN.F.F63433). P. A-1, external view of a left valve, sample 4 (GM5) (MNHN.F.F63434). Q. Paratype, A-1, external view of a left valve, sample 4 (GM5) (MNHN.F.F63435). R. A-1, external view of a left valve, sample 4 (GM5) (MNHN.F.F63436). Scale bars: $100 \mu \mathrm{m}$. 


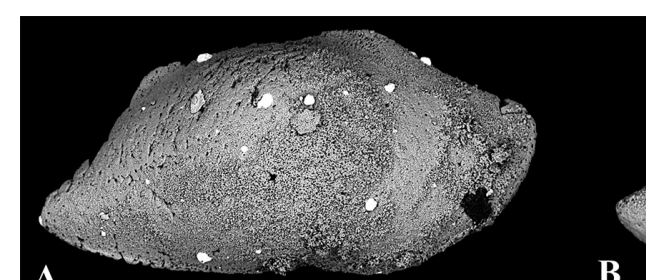

A

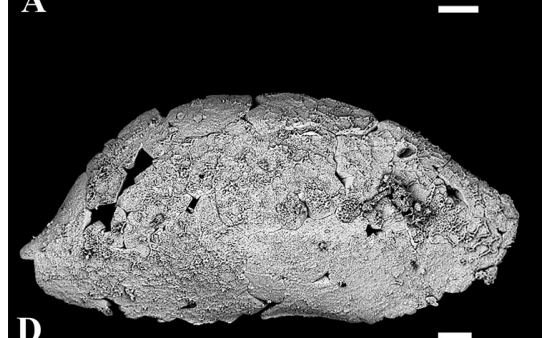

D

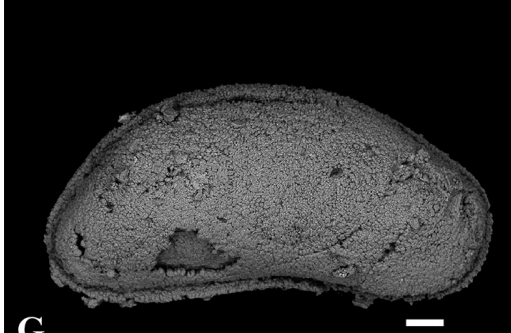

G

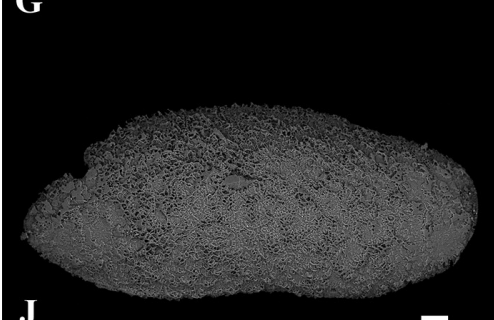

$\mathrm{J}$

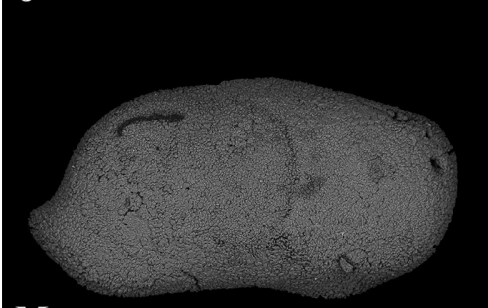

M

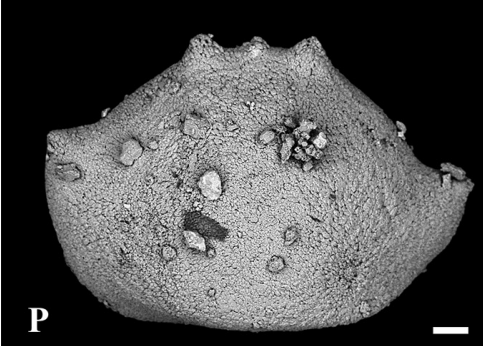

H

K
B

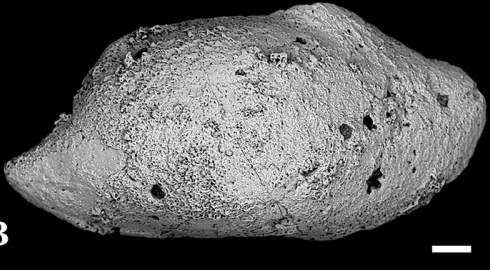

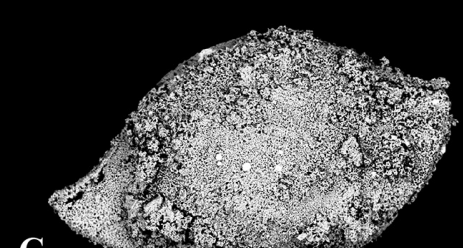

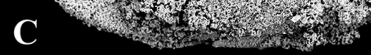

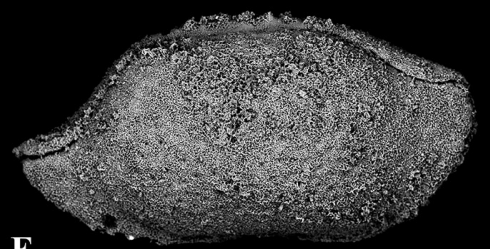

一

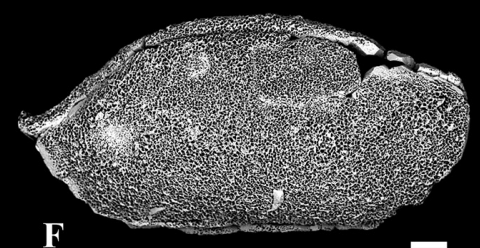

I
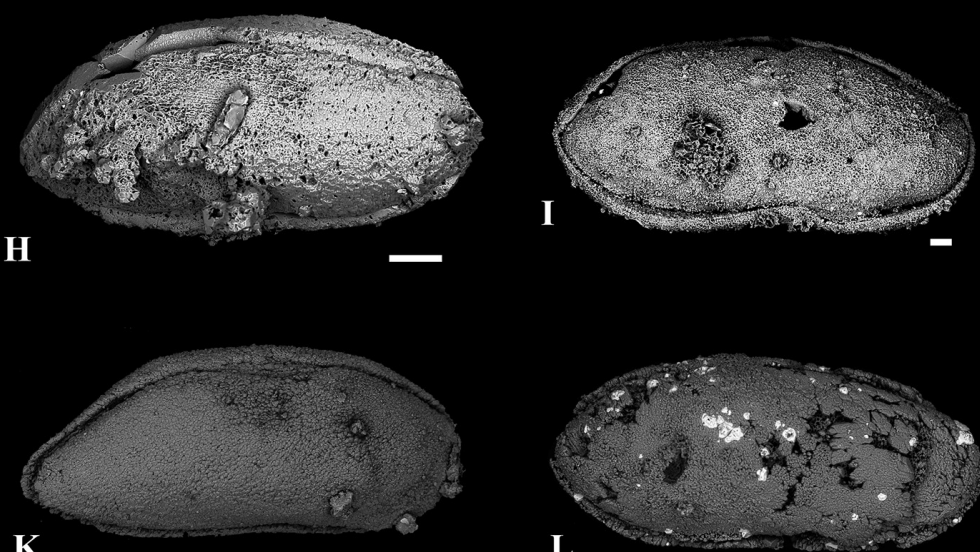

-

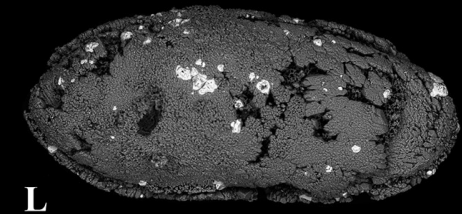

$\mathbf{0}$

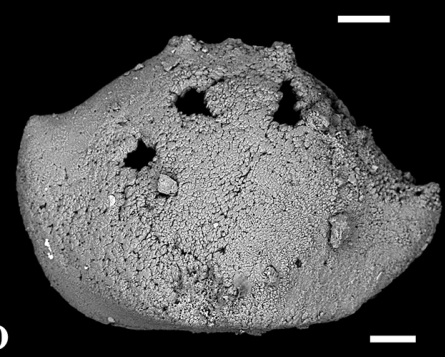

Q
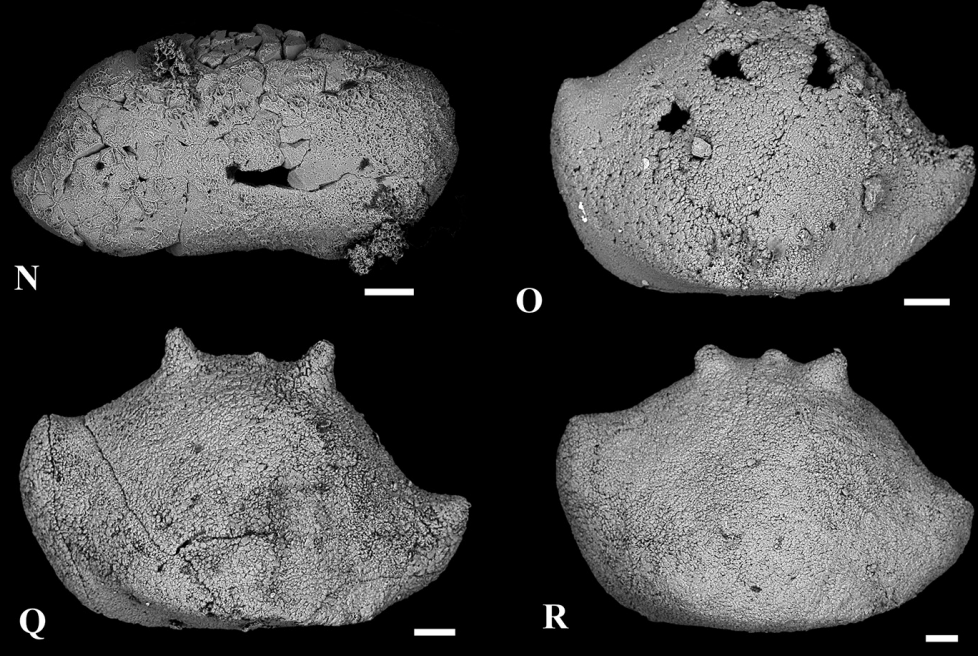


\section{Remarks}

Bairdia sp. A is extremely similar to Bairdia salemensis Geis, 1932 from the Mississippian of Indiana (Geis 1932) that was later considered as a junior synonym of Bairdia permagna Geis, 1932 by Sohn (1960). Sohn (1960) more precisely stated that the type specimens of Bairdia salemensis are young juveniles of Bairdia permagna, although dimensions provided by Geis for Bairdia salemensis are of Lmean $=1450 \mu \mathrm{m}$, Hmean $=685 \mu \mathrm{m}$. In the present analysis, Bairdia sp. A is morphologically closer to specimens considered as young juveniles of Bairdia permagna than to adults and their dimensions are very similar to those reported for Bairdia salemensis with Lmean $=1500 \mu \mathrm{m}$, Hmean $=500 \mu \mathrm{m}$. Herein, however, all specimens at hand assigned to Bairdia sp. A are not abundant enough to discuss their ontogenetic development and verify the conspecificity of the species.

\section{Occurrence}

Sample GM1, Quarry section, Williams Ranch Member, Cutoff Formation, Guadalupe Mountains, West Texas, USA, Roadian, Middle Permian (this work).

Genus Fabalicypris Cooper, 1946

\section{Type species}

Fabalicypris wileyensis Cooper, 1946 by original designation.

Fabalicypris acetalata (Coryell \& Billings, 1932)

Fig. 8I-J

Bairdia acetalata Coryell \& Billings, 1932: 173, pl. 17 fig. 5.

Fabalicypris acetalata - Sohn 1960 (with all previous synonymy therein): 62.

\section{Material examined}

USA - 1 complete carapace; Guadalupe Mountains, West Texas, Williams Ranch Member, Cutoff Formation, Quarry section, sample GM1; Roadian, Middle Permian; MNHN.F.F63427 • 1 right valve; same locality as for preceding but sample GM4; Roadian, Middle Permian; MNHN.F.F63428 • 9 valves; same locality as for preceding but samples GM1, GM2, and GM4; Roadian, Middle Permian; MNHN.F.F63493.

\section{Occurrence}

Kansas, Texas, Illinois, USA, Pennsylvanian (Coryell \& Billings 1932; Sohn 1960); Nebraska, USA, Permian (Sohn 1960); samples GM1, GM2, and GM4, Quarry section, Williams Ranch Member, Cutoff Formation, Guadalupe Mountains, West Texas, USA, Roadian, Middle Permian (this work).

Fabalicypris glennensis (Harlton, 1927)

Fig. 8K

Bairdia glennensis Harlton, 1927: 210, pl. 33 fig. 10.

Bairdia glennensis - Kellett 1935: 133, pl. 18 figs 4a-e.

Fabalicypris glennensis - Sohn 1960: pl. 3 figs 9-10. — Yi 1993: 62, pl. 3 figs 9-10. — Shi \& Chen 2002: 86, pl. 23 figs $1-2$. 


\section{Material examined}

USA 11 complete carapace; Guadalupe Mountains, West Texas, 'McKittrick Canyon' Member, Bell Canyon Formation, MKCS1 section, sample 4 (GM5); Capitanian, Middle Permian; MNHN.F.F63429.

\section{Occurrence}

Kansas, Oklahoma, USA, Kasimovian, Pennsylvanian (Harlton 1927; Kellett 1935; Sohn 1960); Hubei, China, Kungurian, Early Permian (Yi 1993); Sichuan, China, Changhsingian, Late Permian (Shi \& Chen 2002); sample 4 (GM5), MKCS1 section, 'McKittrick Canyon' Member, Bell Canyon Formation, Guadalupe Mountains, West Texas, USA, Capitanian, Middle Permian (this work).

Genus Orthobairdia Sohn, 1960

\section{Type species}

Bairdia cestriensis Ulrich, 1891 subsequently designated by Sohn (1960).

Orthobairdia texana (Harlton, 1927)

Fig. $8 \mathrm{M}-\mathrm{N}$

Bairdia texana Harlton, 1927: 210, pl. 33 fig. 9.

Bairdia chasae Kellett, 1934: pl. 18 figs 5a-d, pl. 19 figs 5a-d.

Bairdia guadalupiana Hamilton, 1942: 714, pl. 110 figs 5a-b.

Orthobairdia texana - Sohn 1960: 68, 69, pl. 3 fig. 29 with all the previous references therein. - Hoare et al. 1999: 444, figs 3.22-3.25.

\section{Material examined}

USA 11 right valve; Guadalupe Mountains, West Texas, 'McKittrick Canyon' Member, Bell Canyon Formation, MKCS1 section, sample 4 (GM5); Capitanian, Middle Permian; MNHN.F.F63431 • 1 right valve; Guadalupe Mountains, West Texas, Williams Ranch Member, Cutoff Formation, Quarry section, sample GM1; Roadian, Middle Permian; MNHN.F.F63432 • 3 valves; Guadalupe Mountains, West Texas, Williams Ranch Member, Cutoff Formation, Quarry section, sample GM1; Roadian; MNHN.F.F63659 - 10 valves; same locality as for preceding; 'McKittrick Canyon' Member, Bell Canyon Formation, MKCS1 section, samples 4 (GM5) and 2 (GM6), Capitanian; Middle Permian; MNHN.F.F63494.

\section{Occurrence}

Kansas, Texas, Missouri, Oklahoma, Nebraska, Illinois, Ohio, USA, Pennsylvanian (Sohn 1960 and all references therein); Glass Mountains, West Texas, USA, Roadian, Middle Permian (Hamilton 1942); Ischimbaev District (North Ural?), Russia, Early Permian (Sohn 1960); Germany, Permian (Sohn 1960); Appalachian Basin, USA, Pennsylvanian (Hoare et al. 1999); sample GM1, Quarry section, Williams Ranch Member, Cutoff Formation, Roadian; samples 4 (GM5) and 2 (GM6), MKCS1 section, 'McKittrick Canyon' Member, Bell Canyon Formation, Capitanian; Guadalupe Mountains, West Texas, USA, Middle Permian (this work).

Genus Ceratobairdia Sohn, 1954

\section{Type species}

Ceratobairdia dorsospinosa Sohn, 1954 by original designation. 
Ceratobairdia mescaleroella Forel sp. nov. urn:1sid:zoobank.org:act:52B30AEF-EB47-4D12-A0BC-20EC095A2F6F

Figs 5E, 8O-R, 9A-C

\section{Diagnosis}

A species of Ceratobairdia with three spines, two long and one short, along the dorsal margin of LV and the ventro-lateral ala not terminated by spine.

\section{Etymology}

From the tribe of Mescalero Apaches who inhabited parts of the Southwest US and Northwest Mexico.

\section{Material examined}

\section{Holotype}

USA • left valve, (Fig. 8O); Guadalupe Mountains, West Texas, 'McKittrick Canyon' Member, Bell Canyon Formation, MKCS1 section, sample 4 (GM5); Capitanian, Middle Permian; MNHN.F.F63433.

\section{Paratypes}

USA 1 left valve (Fig. 9A); Guadalupe Mountains, West Texas, 'McKittrick Canyon' Member, Bell Canyon Formation, MKCS1 section, sample 4 (GM5); Capitanian, Middle Permian; MNHN.F.F63435 (Fig. 8Q) 1 right valve; same locality and sample as for preceding; Capitanian, Middle Permian; MNHN.F.F63437.

\section{Additional material}

USA - 1 left valve; Guadalupe Mountains, West Texas, 'McKittrick Canyon' Member, Bell Canyon Formation, MKCS1 section, sample 4 (GM5); Capitanian, Middle Permian; MNHN.F.F63434 • 1 left valve; same locality and sample as for preceding; Capitanian, Middle Permian; MNHN.F.F63436 1 left valve; same locality as for preceding but sample 2 (GM6); Capitanian, Middle Permian; MNHN.F.F63438 - 1 left valve; same locality and sample as for preceding; Capitanian, Middle Permian; MNHN.F.F63439

Fig. 9 (next page). SEM micrographs of ostracods from the Middle Permian (Roadian, Quarry section, samples GM1; Capitanian, MKCS1 section, samples 4 (GM5) and 2 (GM6)) of the Guadalupe Mountains (West Texas, USA). All specimens are temporarily housed in the collections of the Muséum national d'histoire naturelle, Paris, France (MNHN). A-C. Ceratobairdia mescaleroella Forel sp. nov. A. Paratype, A-1, external view of a right valve, sample 4 (GM5) (MNHN.F.F63437). B. External view of a left valve, A-2, sample 2 (GM6) (MNHN.F.F63438). C. External view of a right valve, A-2, sample 2 (GM6) (MNHN.F.F63439). - D-J. Ceratobairdia sexagintaduella Forel sp. nov. D. Holotype, right lateral view of a carapace, adult, sample GM1 (MNHN.F.F63440). E. Paratype, external view of a left valve, adult, sample GM1 (MNHN.F.F63441). F. External view of a left valve, A-1, sample GM1 (MNHN.F.F63442). G. Right lateral view of a carapace, A-2, sample GM1 (MNHN.F.F63443). H. External view of a left valve, A-2, sample GM1 (MNHN.F.F63444). I. Right lateral view of a carapace, A-3, sample GM1 (MNHN.F.F63445). J. External view of a left valve, A-3, sample GM1 (MNHN.F.F63446). - K-R. Ceratobairdia wordensis (Hamilton, 1942). K. External view of a left valve, adult, sample GM1 (MNHN.F.F63447). L. External view of a left valve, adult, sample GM1 (MNHN.F.F63448). M. External view of a left valve, A-2?, sample GM1 (MNHN.F.F63449). N. External view of a right valve, sample 4 (GM5) (MNHN.F.F63450). O. External view of a right valve, sample GM1 (MNHN.F.F63497). P. External view of a right valve, sample GM1 (MNHN.F.F63451). Q. External view of a left valve, sample GM1 (MNHN.F.F63452). R. Right lateral view of a carapace, sample 4 (GM5) (MNHN.F.F63453). Scale bars: $100 \mu \mathrm{m}$. 


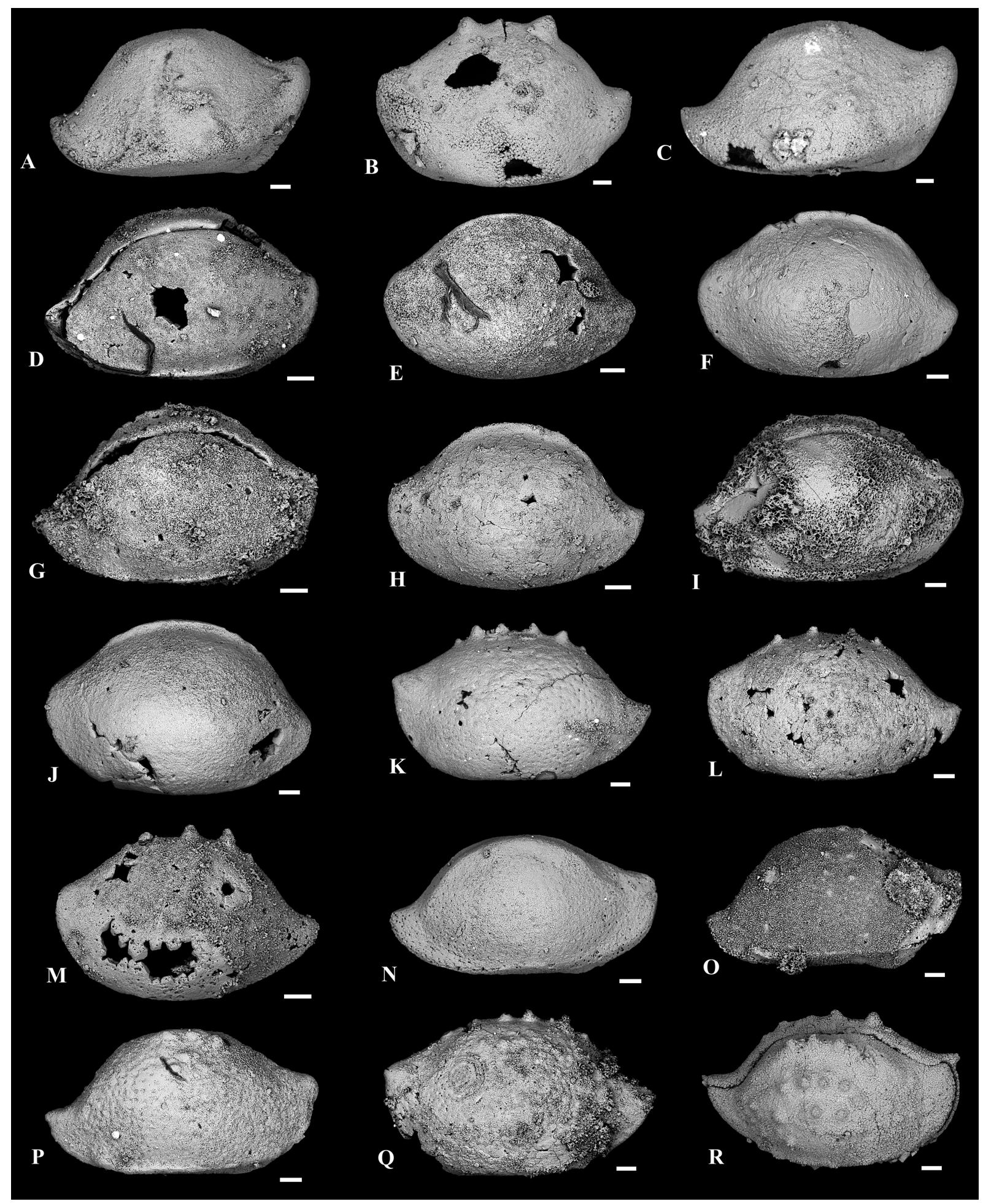


- 13 valves; Guadalupe Mountains, West Texas, 'McKittrick Canyon' Member, Bell Canyon Formation, MKCS1 section, samples 4 (GM5) and 2 (GM6); Capitanian, Middle Permian; MNHN.F.F63495.

\section{Dimensions}

See Fig. 5E.

\section{Description}

LV: stocky, subtriangular in lateral view with Hmax along DB and Lmax in lower half of $\mathrm{H}$; dorsal margin uniformly arched with concave ADB and PDB, and apex of curvature with 3 equidistant spines; the 3 spines triangular in shape and thickest at base, central spine sub-vertical, external spines biggest, anterior one oriented anteriorly and posterior one oriented posteriorly; $\mathrm{AB}$ beak-like located in upper fourth of $\mathrm{H}$ with long and subvertical AVB; ventral margin uniformly convex; PB beak-like, located below mid-H; anterior and posterior margins laterally compressed; ventro-lateral ala delimited by smooth subhorizontal ridge lacking terminal spine.

RV: elongate, subtriangular in lateral view with Hmax at AD angulation and Lmax below mid-H; dorsal margin tripartite with $\mathrm{DB}$ slightly convex, very concave $\mathrm{ADB}$ and $\mathrm{PDB}$; $\mathrm{AB}$ beak-like located in upper fourth of $\mathrm{H}$ with AVB long and straight; $\mathrm{PB}$ beak-like located in lower third of $\mathrm{H}$; ventral margin long and straight except for tenuous oral concavity in anterior part; anterior and posterior margins laterally compressed; lateral surface inflated dorsally into narrow platform and ventrally delimited by lateroventral ala lacking terminal spine and raising upward anteriorly.

\section{Occurrence}

Samples 4 (GM5) and 2 (GM6), MKCS1 section, 'McKittrick Canyon' Member, Bell Canyon Formation, Guadalupe Mountains, West Texas, USA, Capitanian, Middle Permian (this work).

\section{Remarks}

Ceratobairdia dorsospinosa Sohn, 1954 from the Roadian, Middle Permian of Texas (Sohn 1954) is morphologically very similar to Ceratobairdia mescaleroella sp. nov., but the new species differs by its relatively smaller dimensions, three thicker dorsal spines, lack of a terminal sharp spine at the latero-ventral ridge and PB located higher and more upturned. Sohn (1954) observed that specimens of Ceratobairdia dorsospinosa Sohn, 1954 have one or two spines along the dorsal margin of LV. However, all other characters of the carapaces and valves are very similar, so he suggested that all specimens are conspecific. Although very similar morphologically, Ceratobairdia mescaleroella sp. nov. cannot be considered as another degree in the variability of Ceratobairdia dorsospinosa Sohn, 1954 as the lateral morphologies of the two species are significantly different as detailed above. Contrary to what was shown for Ceratobairdia dorsospinosa Sohn, 1954, Ceratobairdia mescaleroella sp. nov. has a conservative number and position of dorsal spines, the only difference observed is in the expression of the central spine.

Ceratobairdia sexagintaduella Forel sp. nov. urn:1sid:zoobank.org:act:E353B465-EF88-46B2-A3E0-5C7C3B2AB411

Figs 5F, 9D-J

\section{Diagnosis}

A species of Ceratobairdia with a lamellar overlap of LV over RV and a curved latero-ventral ridge delimiting a flat venter lacking a posterior spine. 


\section{Etymology}

From the Latin 'sexaginta duo', meaning ' 62 ', referring to the location of the studied sections along the Highway 62.

\section{Material examined}

\section{Holotype}

USA - complete carapace, (Fig. 9D); Guadalupe Mountains, West Texas, Williams Ranch Member, Cutoff Formation, Quarry section, sample GM1; Roadian, Middle Permian; MNHN.F.F63440.

\section{Paratype}

USA - 1 left valve, (Fig. 9E); Guadalupe Mountains, West Texas, Williams Ranch Member, Cutoff Formation, Quarry section, sample GM1; Roadian, Middle Permian; MNHN.F.F63441.

\section{Additional material}

USA - 1 left valve; Guadalupe Mountains, West Texas, Williams Ranch Member, Cutoff Formation, Quarry section, sample GM1; Roadian, Middle Permian; MNHN.F.F63442 • 1 complete carapace; same locality as for preceding; Roadian, Middle Permian; MNHN.F.F63443 • 1 left valve; same locality as for preceding; Roadian, Middle Permian; MNHN.F.F63444 1 complete carapace; same locality as for preceding; Roadian, Middle Permian; MNHN.F.F63445 • 1 left valve; same locality as for preceding; Roadian, Middle Permian; MNHN.F.F63446 29 complete carapaces, 368 valves; Guadalupe Mountains, West Texas, Williams Ranch Member, Cutoff Formation, Quarry section, samples GM1, GM2; Roadian, Middle Permian; MNHN.F.F63496.

\section{Dimensions}

$\mathrm{L}=753-1488 \mu \mathrm{m} ; \mathrm{H}=389-925 \mu \mathrm{m}$ (Fig. 5F, not all valves measured, all RV correspond to complete carapaces).

\section{Description}

Massive carapace $(\mathrm{H} / \mathrm{L}=0.58-0.71)$; AB with small radius of curvature and maximum located at mid- $\mathrm{H}$, dorsal part of $\mathrm{AB}$ forms a right angle with $\mathrm{ADB}$ at $\mathrm{RV}$; VB straight at $\mathrm{RV}$ and convex at $\mathrm{LV}$; $\mathrm{PB}$ short, with small radius of curvature located in lower $1 / 3^{\text {rd }}$ of Hmax; latero-ventral ridge developed along the VB; strong overlap of LV on RV particularly at DB developed into blade; surface smooth.

\section{Occurrence}

Samples GM1, GM2, Quarry section, Williams Ranch Member, CutoffFormation, Guadalupe Mountains, West Texas, USA, Roadian, Middle Permian (this work).

\section{Remarks}

Ceratobairdia sexagintaduella sp. nov. is similar to Bairdia monstrabilis Cooper, 1946 from the Pennsylvanian of Illinois (Cooper 1946) but differs by a shorter carapace, a shorter latero-ventral ridge and the presence of a blade along the dorsal overlap at LV. Noteworthy, Sohn (1954) considered that Bairdia monstrabilis Cooper, 1946 does not belong to Ceratobairdia mainly because of the lack of a dorsal structure at LV. Lobobairdia ventriconcava (Chen, 1958) in Chen \& Bao, 1986 from the Early Permian of South China (Chen \& Bao 1986) is similar to Ceratobairdia sexagintaduella sp. nov., but has an even shorter latero-ventral ridge and lacks the dorsal blade at LV. The morphology of the latero-ventral ridge in Ceratobairdia sexagintaduella sp. nov. is similar to that of Ceratobairdia sinensis Wang, 1978 from the Changhsingian, Late Permian of South China (Wang 1978) by the morphology of the lateroventral ridge but differs by lamellar DB at LV, PB less upturned and AB located lower. Ceratobairdia sexagintaduella sp. nov. is very similar to Bairdia permiana Hamilton, 1942 from the Kungurian of the 
Glass Mountains (Hamilton 1942): this species has later been shown to be a junior synonym of Bairdia pecosensis Delo, 1930 by Sohn (1960), who additionally mentioned that the ridge on the ventral part of the RV is not as pronounced as shown in the original drawing of Hamilton (1942).

Ceratobairdia wordensis (Hamilton, 1942)

Figs $9 \mathrm{~K}-\mathrm{R}, 10$

Bairdia wordensis Hamilton, 1942: 716, pl. 110 fig. 4.

Ceratobairdia wordensis - Sohn 1954: 5; 1960: 69, pl. 4 figs 8-17.

\section{Material examined}

USA - 1 left valve; Guadalupe Mountains, West Texas, Williams Ranch Member, Cutoff Formation, Quarry section, sample GM1; Roadian, Middle Permian; MNHN.F.F63447 • 1 left valve; same locality as for preceding; Roadian, Middle Permian; MNHN.F.F63448 • 1 left valve; same locality as for preceding; Roadian, Middle Permian; MNHN.F.F63449 - 1 right valve; Guadalupe Mountains, West Texas, 'McKittrick Canyon' Member, Bell Canyon Formation, MKCS1 section, sample 4 (GM5); Capitanian, Middle Permian; MNHN.F.F63450 • 1 right valve; Guadalupe Mountains, West Texas, Williams Ranch Member, Cutoff Formation, Quarry section, sample GM1; Roadian, Middle Permian; MNHN.F.F63497 - 1 right valve; same locality as for preceding; Roadian, Middle Permian; MNHN.F.F63451 • 1 left valve; same locality as for preceding; Roadian, Middle Permian; MNHN.F.F63452 - 1 complete carapace; Guadalupe Mountains, West Texas, 'McKittrick Canyon' Member, Bell Canyon Formation, MKCS1 section, sample 4 (GM5); Capitanian, Middle Permian; MNHN.F.F63453 • 1 broken carapace, 20 left valves; Guadalupe Mountains, West Texas, Williams Ranch Member, Cutoff Formation, Quarry section, samples GM1, GM2, and GM3; Roadian; MNHN.F.F63660 3 broken carapaces, 15 left valves; same locality as for preceding; 'McKittrick Canyon' Member, Bell Canyon Formation, MKCS1 section, samples 4 (GM5) and 2 (GM6), Capitanian; Middle Permian; MNHN.F.F63498.

\section{Dimensions}

$\mathrm{L}=727-1450 \mu \mathrm{m} ; \mathrm{H}=397-920 \mu \mathrm{m}$ (Fig. 10, not considering spines).

\section{Occurrence}

Glass Mountains, Texas, USA, Roadian, Middle Permian (Hamilton 1942; Sohn 1954, 1960); samples GM1, GM2, and GM3, Quarry section, Williams Ranch Member, Cutoff Formation, Roadian; samples 4 (GM5) and 2 (GM6), MKCS1 section, 'McKittrick Canyon' Member, Bell Canyon Formation, Capitanian; Guadalupe Mountains, West Texas, USA, Middle Permian (this work).

\section{Remarks}

Most of the specimens of Ceratobairdia wordensis (Hamilton, 1942) in the present work occur as isolated valves and LV are relatively easily assigned to this species based on their unique dorsal ornamentation. The identification of RV is more complex and has been possible because of the description provided by Hamilton (1942: 716) of "the centrodorsal portion of the right valve flattens abruptly to hingeline, expressed as a flat terrace in dorsal bi and as a rather prominent ridge in lateral view; [...]; the ventral portion of the right valve sharply infolded, forming a sharp downward projecting ridge terminating in mature molds as a backward projecting spine."

Hamilton (1942: 716) described the dorsal margin of LV of Ceratobairdia wordensis as "usually ornamented by two to seven short strong spines equally spaced and concentrated dorsally on the middle third or more." The present material provided LV with 4 to 6 spines, but all other characters leave no doubt on the conspecificity of the specimens. Is the number of spines related to ontogeny? Although 

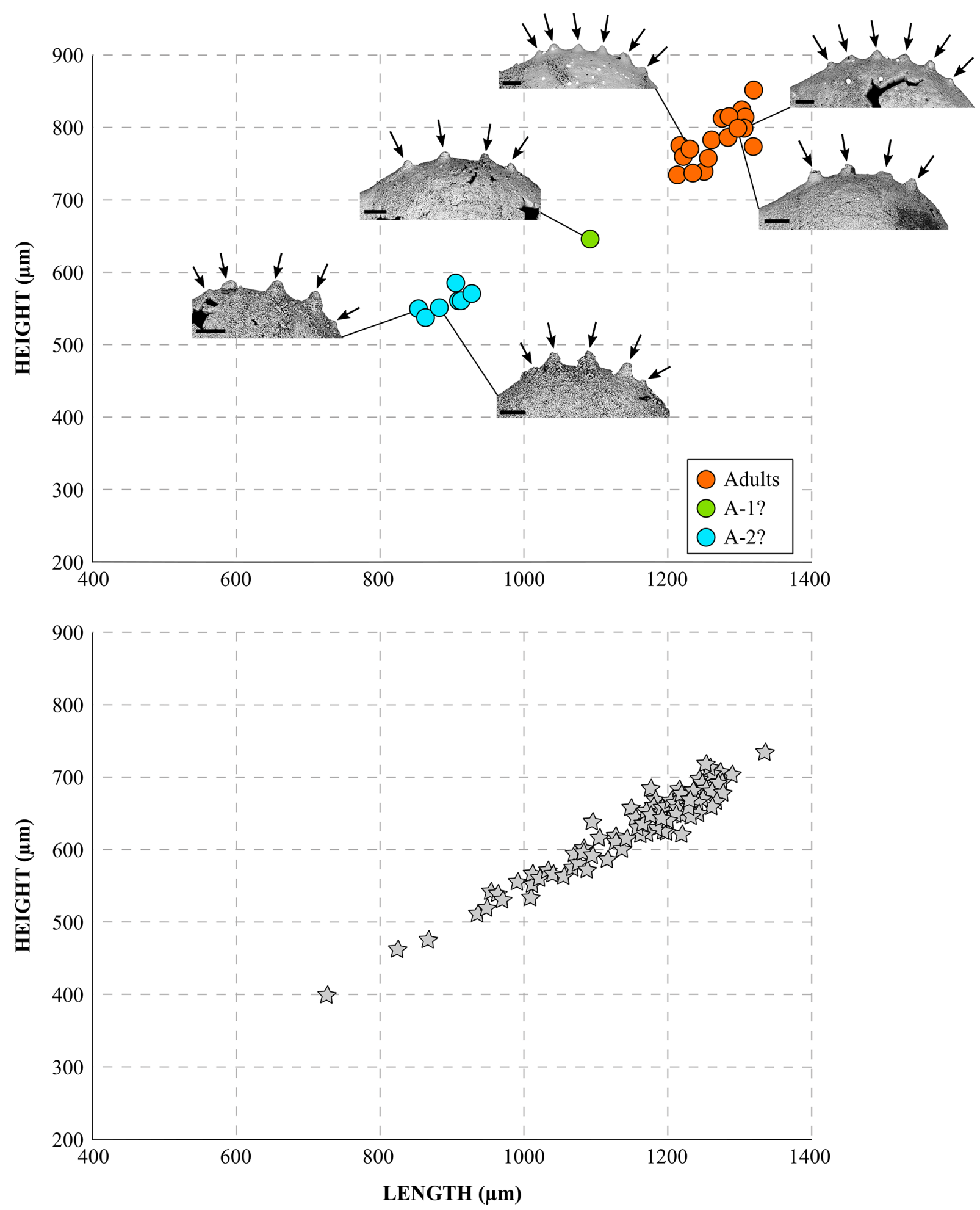

Fig. 10. Height/length scatter plots of Ceratobairdia wordensis (Hamilton, 1942) from the Middle Permian (Roadian, Capitanian) of the Guadalupe Mountains (West Texas, USA). Top: left valves (arrows pointing to dorsal spines), bottom: right valves. 
Hamilton (1942) mentioned 2 to 7 dorsal spines on LV, the unique specimen illustrated displays 6 spines and no discussion is provided on the possible link between size and number of the spines. Conversely, Sohn (1960: pl. 4 figs 12,14, 17) illustrated specimens with 8 to 10 dorsal spines and at least one of them shows spines organized into 2 overlapping rows. For the present material, the number of spines along the dorsal margin of LV of Ceratobairdia wordensis ranges from 4 to 6 without pattern related to ontogeny (Fig. 10):

- most of the smallest specimens, assigned to A-2?, are weathered but the two sufficiently well preserved at dorsal margin show 5 spines, the 2 external being 'embryonic',

- the only known specimen of A-1? stage displays 4 dorsal spines,

- the adult specimens display from 4 to 6 spines, the external ones being the smallest.

It is worth noting that in A-2? specimens, the external spines are very small and may be overlooked, leading to the wrong identification of only 3 dorsal spines. However, these specimens cannot be confused with Petasobairdia tricornuta Chen in Shi \& Chen, 2002 from the Late Permian of South China (Shi \& Chen 2002) which is more elongate, larger, and with only 3 spines for specimens of a size slightly larger than the adults of Ceratobairdia wordensis.

Asignificant morphological trend is observed in Ceratobairdia wordensis from the Guadalupe Mountains: whereas the majority of specimens have a relatively smooth surface, several show the development of scattered nodules in the dorsal area of their lateral surface (Fig. 9O-Q). This trend culminates in very rare specimens with the entire lateral surface of the valves covered by nodules (Fig. 9R). This nodular surface is reminiscent of Pustulobairdia Sohn, 1960 but all characters of Ceratobairdia wordensis and the morphological trend observed from smooth to nodular leave no doubt that these specimens belong to Ceratobairdia wordensis.

Ceratobairdia pratti Forel sp. nov. urn:lsid:zoobank.org:act:97132C11-D593-4D25-AFA4-05B453873EA4

Figs $5 \mathrm{G}, 11 \mathrm{C}-\mathrm{G}$

\section{Diagnosis}

A species of Ceratobairdia with a nodular dorsal blade at LV displaying 6 very small triangular nodules?, $\mathrm{AB}$ and $\mathrm{PB}$ prolonged by short horizontal spines, posterior end reduced.

\section{Etymology}

Named after the geologist Wallace Pratt who donated a part of the McKittrick Canyon which became part of the Guadalupe Mountains National Park, Guadalupe Mountains, West Texas, USA.

\section{Material examined}

\section{Holotype}

USA - complete carapace (Fig. 11C); Guadalupe Mountains, West Texas, Williams Ranch Member, Cutoff Formation, Quarry section, sample GM1; Roadian, Middle Permian; MNHN.F.F63456.

\section{Paratype}

USA • 1 left valve, (Fig. 11D); Guadalupe Mountains, West Texas, Williams Ranch Member, Cutoff Formation, Quarry section, sample GM1; Roadian, Middle Permian; MNHN.F.F63457. 
Additional material

USA - 1 left valve; Guadalupe Mountains, West Texas, Williams Ranch Member, Cutoff Formation, Quarry section, sample GM1; Roadian, Middle Permian; MNHN.F.F63458 • 1 left valve; same locality as for preceding; Roadian, Middle Permian; MNHN.F.F63459 1 left valve; same locality as for preceding; Roadian, Middle Permian; MNHN.F.F63460 - 43 left valves; Guadalupe Mountains, West Texas, Williams Ranch Member, Cutoff Formation, Quarry section, samples GM1, GM2; Roadian, Middle Permian; MNHN.F.F63499.

\section{Dimensions}

$\mathrm{L}=981-1317 \mu \mathrm{m} ; \mathrm{H}=607-846 \mu \mathrm{m}$ (Fig. 5G, only complete left valves measured, not considering spines).

\section{Description}

Sub-rectangular in lateral outline with Hmax around mid-L and Lmax around mid-H; dorsal margin uniformly convex with ADB straight and short; strong overlap of LV on RV along entire dorsal margin, much narrower along venter; PB steep, short and concave; dorsal margin transformed into nodular blade with 6 small triangular nodules; $\mathrm{AB}$ large with maximum of curvature around upper $1 / 3^{\text {rd }}$ and AVB plump, very convex; ventral margin straightly to slightly concave around mid-L; PB reduced, relatively large with maximum of curvature around mid- $\mathrm{H} ; \mathrm{AB}$ and $\mathrm{PB}$ slightly compressed laterally, prolonged by thick, short horizontal spines; surface smooth.

\section{Occurrence}

Samples GM1, GM2, Quarry section, Williams Ranch Member, CutoffFormation, Guadalupe Mountains, West Texas, USA, Roadian, Middle Permian (this work).

\section{Remarks}

Ceratobairdia pratti sp. nov. is unique among species of Ceratobairdia known to date by the disposition of nodules along a nodular blade at LV and by the spines terminating AB and PB.

Genus Petasobairdia Chen in Chen \& Shi, 1982

Praelobobairdia Kozur, 1991: 66.

\section{Type species}

Petasobairdia bicornuta Chen in Chen \& Shi, 1982 by original designation.

Petasobairdia campbelli Chitnarin in Chitnarin et al., 2017

Fig. $11 \mathrm{H}-\mathrm{I}$

Petasobairdia campbelli Chitnarin in Chitnarin et al., 2017: 670, figs 12j-p.

\section{Material examined}

USA - 1 left valve; Guadalupe Mountains, West Texas, Williams Ranch Member, Cutoff Formation, Quarry section, sample GM2; Roadian, Middle Permian; MNHN.F.F63461 • 1 left valve; same locality as for preceding but sample GM4; Roadian, Middle Permian; MNHN.F.F63462.

\section{Occurrence}

Central Thailand, Artinskian, Roadian?, Early-Middle Permian (Chitnarin et al. 2017); samples GM2, GM4, Quarry section, Williams Ranch Member, Cutoff Formation, Guadalupe Mountains, West Texas, USA, Roadian, Middle Permian (this work). 
Superfamily Sigillioidea Mandelstam, 1960

Family Microcheilinellidae Gramm, 1975

Genus Microcheilinella Geis, 1933

\section{Type species}

Microcheilus distortus Geis, 1932 by original designation.

\section{Microcheilinella postspinosa Chen, 1958}

Fig. 11L

Microcheilinella postspinosa Chen, 1958: 254, pl. 2 figs 1-6.

Microcheilinella postspinosa - Chen \& Bao 1986: 110.

non Microcheilinella postspinosa - Crasquin-Soleau \& Baud 1998: 146, pl. 7 figs 5-9.

\section{Material examined}

USA 11 complete carapace; Guadalupe Mountains, West Texas, 'McKittrick Canyon' Member, Bell Canyon Formation, MKCS1 section, sample 2 (GM6); Capitanian, Middle Permian; MNHN.F.F63465 - 1 left valve; same locality as for preceding but sample 4 (GM5); Capitanian, Middle Permian; MNHN.F.F63472.

\section{Occurrence}

Chishia Limestone, Kwanshan, Lungtan, China, Kungurian, Early Permian (Chen 1958); Jurong, China, Kungurian, Early Permian (Chen \& Bao 1986); samples 4 (GM5) and 2 (GM6), MKCS1 section,

Fig. 11 (next page). SEM micrographs of ostracods from the Middle Permian (Roadian, Quarry section, samples GM1, GM2 and GM4; Capitanian, MKCS1 section, samples 4 (GM5) and 2 (GM6)) of the Guadalupe Mountains (West Texas, USA), except R and S from the late Capitanian, Middle Permian, Penglaitan section, Guangxi, South China (Zazzali 2016, unpublished PhD thesis). All specimens from A-Q are temporarily housed in the collections of the Muséum national d'histoire naturelle, Paris, France (MNHN), specimens R and S are housed in the collections of the Sorbonne University, Paris, France. A-B. Ceratobairdia $\mathrm{cf}$. ventrocostata Wang, 1978. A. External view of a left valve, sample 4 (GM5) (MNHN.F.F63454). B. External view of a left valve, sample 2 (GM6) (MNHN.F.F63455). C-G. Ceratobairdia pratti Forel sp. nov. C. Holotype, right lateral view of a carapace, sample GM1 (MNHN.F.F63456). D. Paratype, external view of a left valve, sample GM1 (MNHN.F.F63457). E. External view of a left valve, sample GM1 (MNHN.F.F63458). F. External view of a left valve, sample GM1 (MNHN.F.F63459). G. External view of a left valve, sample GM1 (MNHN.F.F63460). - H-I. Petasobairdia campbelli Chitnarin in Chitnarin et al., 2017. H. External view of a left valve, sample GM2 (MNHN.F.F63461). I. External view of a left valve, sample GM4 (MNHN.F.F63462). - J. Petasobairdia cf. bicornuta Chen in Chen \& Shi, 1982, right lateral view of a carapace, sample 4 (GM5) (MNHN.F.F63463). - K. Petasobairdia? sp., external view of a right valve, sample 4 (GM5) (MNHN.F.F63464). - L. Microcheilinella postspinosa Chen, 1958, right lateral view of a carapace, sample 2 (GM6) (MNHN.F.F63465). - M. Microcheilinella sp. 15 in Zazzali et al. (2015), right lateral view of a carapace, sample 4 (GM5) (MNHN.F.F63466). - N-O. Microcheilinella sp. N. Right lateral view of a carapace, sample 2 (GM6) (MNHN.F.F63467). O. Right lateral view of a carapace, sample 4 (GM5) (MNHN.F.F63468). - P-Q. Denticupachydomella bellcanyonensis Forel sp. nov. P. Holotype, right lateral view of a carapace, sample 4 (GM5) (MNHN.F.F63469). Q. Paratype, external left of a right valve, sample 2 (GM6) (MNHN.F.F63470). - R-S. Microcheilinella sp. D in Zazzali (2016). R. Dorsal view of a carapace (P6M 3778). S. Right lateral view of a carapace (P6M 3779). Scale bars: $100 \mu \mathrm{m}$. 

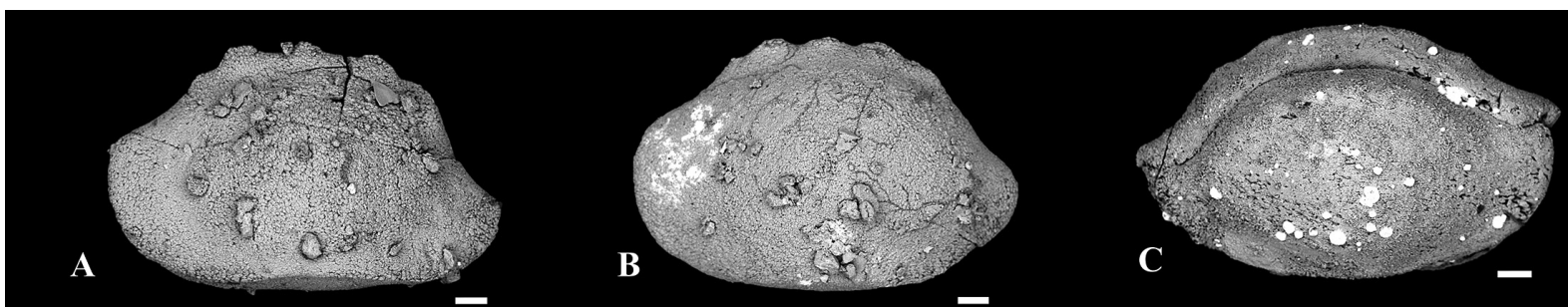

D
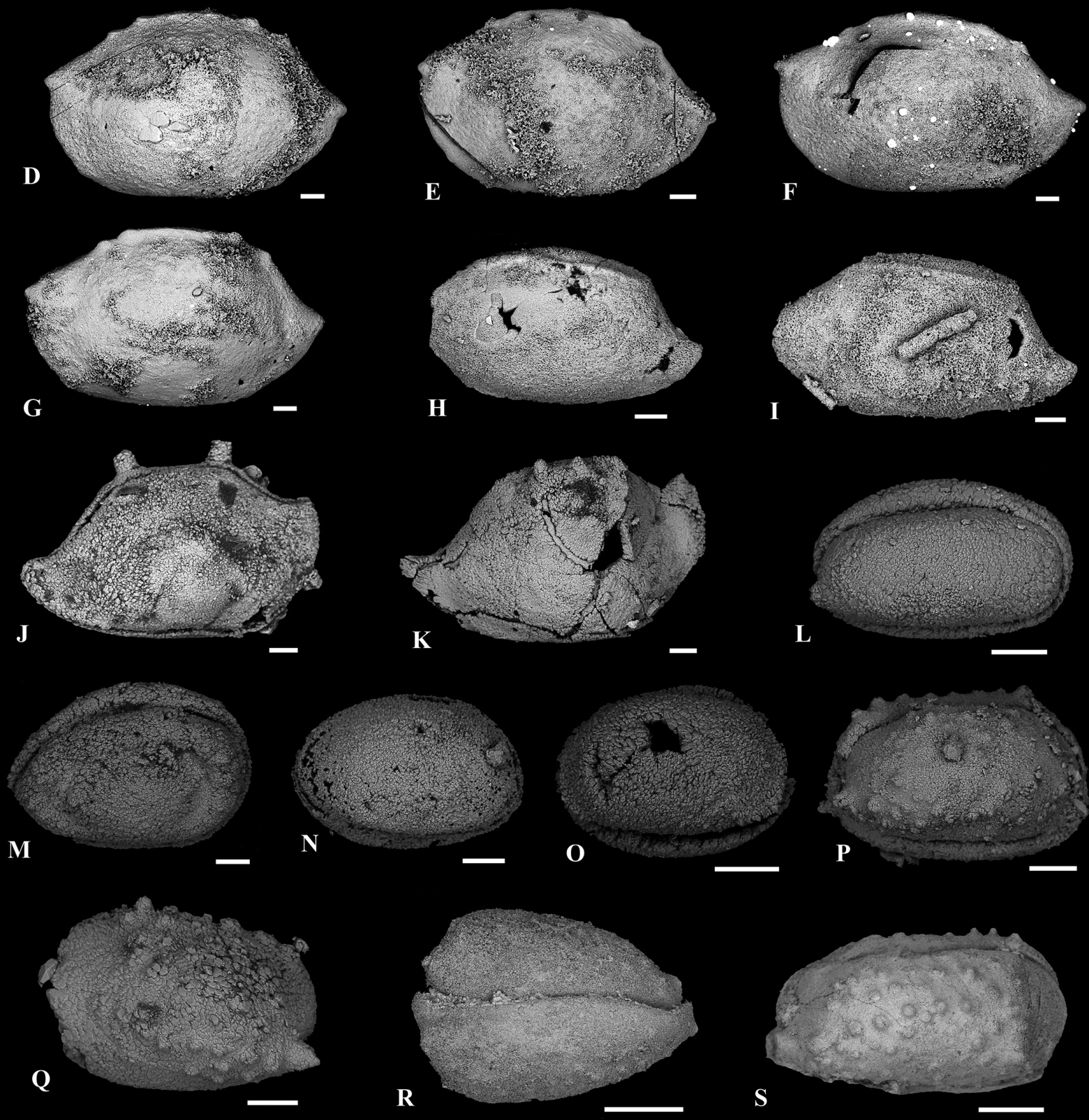
'McKittrick Canyon' Member, Bell Canyon Formation, Guadalupe Mountains, West Texas, USA, Capitanian, Middle Permian (this work).

Microcheilinella sp. 15 in Zazzali et al. 2015

Fig. $11 \mathrm{M}$

Microcheilinella sp. 15 - Zazzali et al. 2015: fig. 12m.

\section{Material examined}

USA - 1 complete carapace; Guadalupe Mountains, West Texas, 'McKittrick Canyon' Member, Bell Canyon Formation, MKCS1 section, sample 4 (GM5); Capitanian, Middle Permian; MNHN.F.F63466 - 1 complete carapace, 3 left valves; same locality as for preceding but samples 4 (GM5) and 2 (GM6); Capitanian, Middle Permian; MNHN.F.F63471.

\section{Occurrence}

Chaotian section, Sichuan, China, Wuchiapingian, Late Permian (Zazzali et al. 2015); samples 4 (GM5) and 2 (GM6), MKCS1 section, 'McKittrick Canyon' Member, Bell Canyon Formation, Guadalupe Mountains, West Texas, USA, Capitanian, Middle Permian (this work).

\section{Remarks}

The carapace of this species is subtriangular in lateral view, with large AB and strong overlap of LV on $\mathrm{RV}$, hemi-circular furrows are visible on the medio-anterior and medio-posterior parts of the carapace in both valves.

Genus Denticupachydomella Yuan in Yuan et al., 2009

\section{Type species}

Denticupachydomella spinosa Yuan in Yuan et al., 2009 by original designation.

\section{Original diagnosis}

"A new Pachydomellidae genus with left valve having straight dorsal border, two horny dorsal spines erecting at the anterior cardinal angle, one stronger spine at the posterior cardinal angle, three denticles along dorsal border, one posteroventral spine and pustulous lateral surface." (from Yuan et al. 2009: 392-393).

\section{Emended diagnosis}

A new genus with a relatively massive postero-ventral spine, spines of various morphology and strength developed along the dorsal margin of the left valve and surface pustulose.

\section{Assigned species}

The following species are herein re-assigned to the genus Denticupachydomella:

Microcheilinella? multinodosa Forel in Crasquin et al., 2010 from the Changhsingian, Late Permian of the Meishan section, Zhejiang Province, China (Crasquin et al. 2010);

Microcheilinella sp. D in Zazzali (2016: 208-209, pl. 14f-g, unpublished material, PhD thesis), Late Capitanian, Middle Permian, Penglaitan section, Guangxi, South China (Fig. 11R-S). 


\section{Remarks}

The thorough comparison of ornate Microcheilinellidae revealed that all the above-mentioned species share postero-ventral spines on both valves and spines/nodules/pustules along the dorsal margin and over the lateral surface. Conversely, the two horny dorsal spines and the three denticles along the dorsal border originally described by Yuan et al. (2009) as of generic significance to the newly erected genus Denticupachydomella rather appear as variations of the morphology and strength of the spines, thus being of specific significance. The diagnosis of Denticupachydomella Yuan in Yuan et al., 2009 is here emended to clarify this issue of generic versus specific characters.

Denticupachydomella bellcanyonensis Forel sp. nov. urn:1sid:zoobank.org:act:89B99A6B-AD6C-43DD-BAAD-D85F291FCBD3

Fig $5 \mathrm{H}, 11 \mathrm{P}-\mathrm{Q}$

\section{Diagnosis}

A species of Denticupachydomella with a row of nodules uniform in size along DB of LV and a surface ornamented by nodules.

\section{Etymology}

After the Bell Canyon Formation (Middle Permian, West Texas) that yielded this new species.

\section{Material examined}

\section{Holotype}

USA - complete carapace, (Fig. 11P); Guadalupe Mountains, West Texas, 'McKittrick Canyon' Member, Bell Canyon Formation, MKCS1 section, sample 4 (GM5); Capitanian, Middle Permian; MNHN.F.F63469.

\section{Paratype}

USA 1 left valve, (Fig. 11Q); Guadalupe Mountains, West Texas, 'McKittrick Canyon' Member, Bell Canyon Formation, MKCS1 section, sample 2 (GM6); Capitanian, Middle Permian; MNHN.F.F63470.

\section{Additional material}

USA - 1 complete carapace, 13 valves; Guadalupe Mountains, West Texas, 'McKittrick Canyon' Member, Bell Canyon Formation, MKCS1 section, samples 4 (GM5) and 2 (GM6); Capitanian, Middle Permian; MNHN.F.F63500.

\section{Dimensions}

$\mathrm{L}=387-756 \mu \mathrm{m} ; \mathrm{H}=247-433 \mu \mathrm{m}$ (Fig. 5H).

\section{Description}

Small carapace sub-rectangular, $\mathrm{H} / \mathrm{L}=0.57-0.69$; $\mathrm{DB}$ straight on both valves; row of nodules long $\mathrm{DB}$ of LV; $\mathrm{AB}$ with quite large radius of curvature with maximum located below mid-H; VB almost straight at both valves; PB with large radius of curvature for genus with maximum of curvature located in lower part of $\mathrm{H}$; presence of spine in posteroventral part of carapace; overlap of LV on RV all around carapace; presence of nodules on surface of both valves.

\section{Occurrence}

Samples 4 (GM5) and 2 (GM6), MKCS1 section, 'McKittrick Canyon' Member, Bell Canyon Formation, Guadalupe Mountains, West Texas, USA, Capitanian, Middle Permian (this work). 


\section{Remarks}

Denticupachydomella bellcanyonensis sp. nov. is very similar to the type species Denticupachydomella spinosa Yuan in Yuan et al., 2009 from the Changhsingian of South China (Yuan et al. 2009) but is larger and lacks the two horny spines at the anterior and posterior cardinal angles. Denticupachydomella bellcanyonensis sp. nov. differs from Denticupachydomella multinodosa (Forel in Crasquin et al. 2010) from the Changhsingian (Late Permian) of Sichuan, south-west China (Crasquin et al. 2010) by the presence of a postero-ventral spine and by the organisation of nodules along dorsal and ventral margins at both valves. Herein, the dorso-central shoulder at RV is absent and the surface is covered by nodules. The species from the Late Capitanian of the Penglaitan section (Guadalupuan-Lopingian GSSP) in Guangxi, South China (Zazzali 2016: fig. 13o-p, unpublished material) differs from Denticupachydomella bellcanyonensis sp. nov. by a more elongate carapace and a PB with smaller radius of curvature. The species from the Penglaitan section is also new and has to be described.

\section{Discussion}

\section{Diversity and taxonomic composition of ostracod assemblages}

Each of the 6 samples studied from the Middle Permian (Roadian and Capitanian) of the Guadalupe Mountains, West Texas, USA, yielded identifiable ostracods. In total, 51 species have been identified, belonging to 26 genera and 15 families. Seventeen species were previously known from the Permian, 11 are new and 23 are kept in open nomenclature due to poor preservation and/or paucity of material (including Microcheilinella sp. 15 in Zazzali et al. 2015, which occurs in the Wuchiapingian, Late Permian of South China). Table 1 provides a complete taxonomic list of the two assemblages from the Middle Permian (Roadian and Capitanian) of the Guadalupe Mountains. The assemblages described in the present contribution are obtained from two continuous bedded sections.

The diversity level of the Roadian assemblage is slightly higher with 34 species, 21 genera, and 13 families whereas in the Capitanian assemblage, the ostracods are represented by 26 species, 18 genera, and 11 families. Across the studied area, 23 species are restricted to the Roadian (including Healdia mckittrickensis sp. nov., Amphissites centronotus (Ulrich \& Bassler, 1906), Kindlella fissiloba Sohn, 1954, Aurikirkbya guadalupensis sp. nov., Geisina culbersonensis sp. nov., Acratia sinuata (Kozur, 1991), Bairdia subfusiformis Hamilton, 1942, B. elcapitanensis sp. nov., Fabalicypris acetalata (Coryell \& Billings, 1932), Ceratobairdia sexagintaduella sp. nov., C. pratti sp. nov., and Petasobairdia campbelli Chitnarin in Chitnarin et al., 2017) and 15 are restricted to the Capitanian (including Kindlella bellsundi Olempska \& Błaszyk, 1996, Paraparchites texanus Delo, 1930, Bairdia rhomboidalis Hamilton, 1942, Fabalicypris glennensis (Harlton, 1927), Ceratobairdia mescaleroella sp. nov., Microcheilinella postspinosa Chen, 1958, and Denticupachydomella bellcanyonensis sp. nov.). Conversely, eight genera are restricted to the Roadian (Amphissites, Polytylites, Kirkbya, Glyptopleura, Geisina, Libumella, Fellerites, Acratia) and five are only found in the Capitanian (Kirkbyidae gen. et sp. indet., Kirkbyella, Miltonella, Microcheilinella, Denticupachydomella). At the family level, Amphissitidae, Glyptopleuridae, Geisinidae, Rozhdestvenskaytidae are restricted to the Roadian whereas Microcheilinidae and Kirkbyellidae are restricted to the Capitanian.

In terms of family composition (Fig. 12), both assemblages are dominated by Bairdiidae (Acratia, Bairdia, Bairdiacypris, Ceratobairdia, Fabalicypris, Orthobairdia, Petasobairdia) that account for 53\% and $42 \%$ of the species in the Roadian and the Capitanian, respectively. The Roadian and Capitanian assemblages differ by their respective composition in secondary and accessory families. In the Roadian, all families except Bairdiidae account for less than $10 \%$ of the species. Of them, Kirkbyidae (Aurikirkbya, Kirkbya) is the most diverse with $9 \%$ of the species. Amphissitidae (Amphissites, Polytylites) and Healdiidae (Healdia) each account for $6 \%$ of the species. Accessory families are Paraparchitidae (Paraparchites), Cavellinidae (Sulcella), Kelletinidae (Kindlella), Scrobiculidae 
Table 1 (continued on next page). Taxonomic list of all ostracod species identified from the Roadian and Capitanian of the Guadalupe Mountains, West Texas, USA.

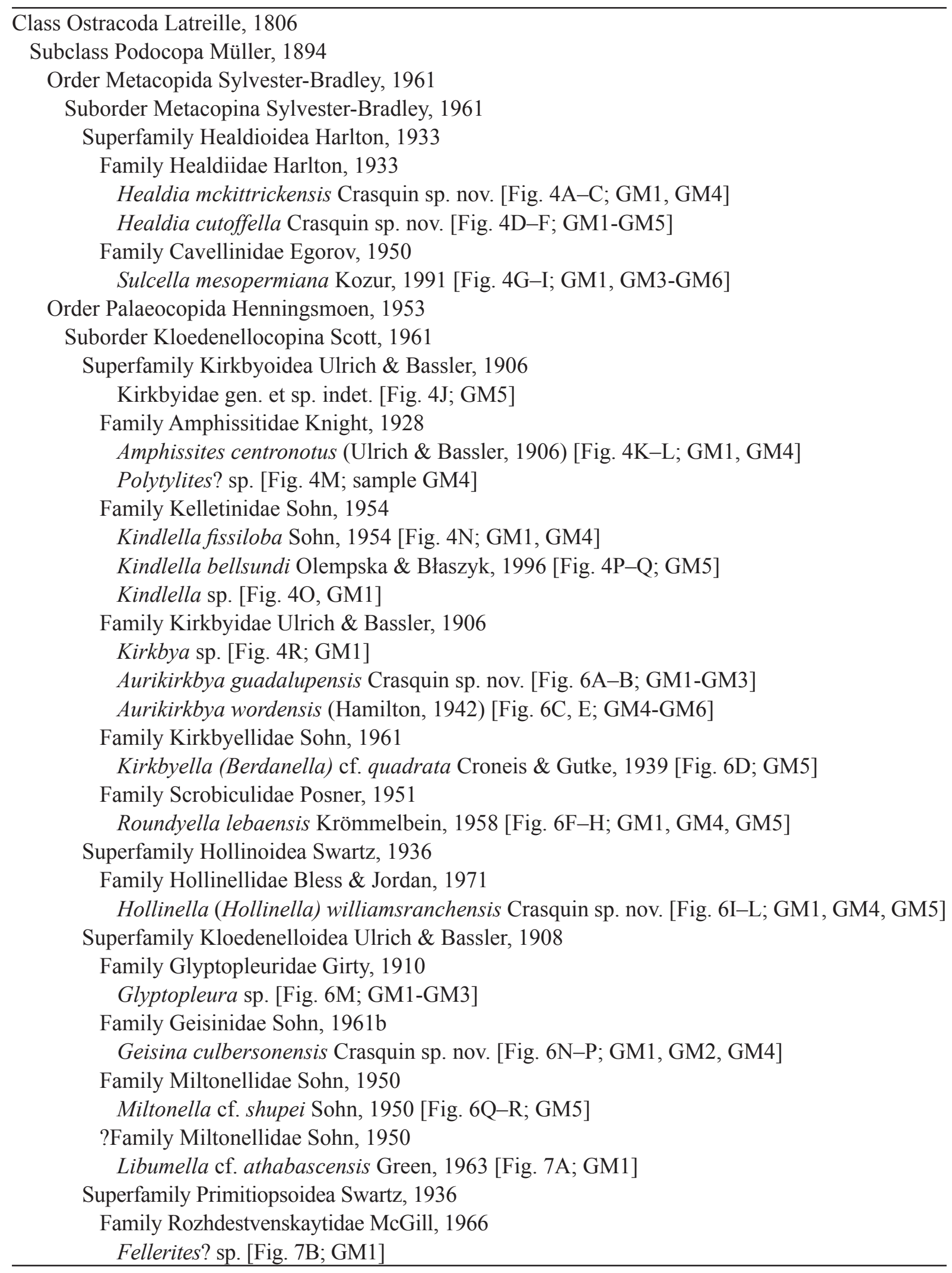


Table 1 (continued). Taxonomic list of all ostracod species identified from the Roadian and Capitanian of the Guadalupe Mountains, West Texas, USA.

Order Leiocopa Schallreuter, 1973

Superfamily Paraparchitoidea Scott, 1959

Family Paraparchitidae Scott, 1959

Paraparchites texanus Delo, 1930 [Fig. 7C; GM5]

Paraparchites pecosensis Crasquin sp. nov. [Fig. 7D-H; GM1-GM3, GM6]

Order Podocopida Sars, 1866

Suborder Podocopina Sars, 1866

Superfamily Bairdioidea Sars, 1887

Family Bairdiidae Sars, 1865

Acratia sp. [Fig. 7I; GM2]

Acratia cf. sinuata (Kozur, 1991) [Fig. 7J; GM1]

Acratia sinuata (Kozur, 1991) [Fig. 7K; GM1, GM4]

Bairdia radlerae Kellett, 1934 [Fig. 7L-M; GM1, GM2, GM4-GM6]

Bairdia rhomboidalis Hamilton, 1942 [Fig. 7N; GM5, GM6]

Bairdia subfusiformis Hamilton, 1942 [Fig. 7O; GM1]

Bairdia elcapitanensis Forel sp. nov. [Figs 7P-R, 8A-B; GM1]

Bairdia cf. postacuta Chen, 1958 [Fig. 8C; GM1, GM2, GM5, GM6]

Bairdia sp. A [Fig. 8D; GM1]

Bairdia sp. B [Fig. 8E; GM1]

Bairdia sp. C [Fig. 8F; GM1]

Bairdiacypris sp. B [Fig. 8G; GM1-GM6]

Bairdiacypris sp. C [Fig. 8H; GM1]

Fabalicypris acetalata (Coryell \& Billings, 1932) [Fig. 8I-J; GM1, GM2, GM4]

Fabalicypris glennensis (Harlton, 1927) [Fig. 8K; GM5]

Fabalicypris sp. [Fig. 8L; GM1, GM2, GM4]

Orthobairdia texana (Harlton, 1927) [Fig. 8M, N; GM1, GM5, GM6]

Ceratobairdia mescaleroella Forel sp. nov. [Fig. 8O-R, 9A-C; GM5, GM6]

Ceratobairdia sexagintaduella Forel sp. nov. [Fig. 9D-J; GM1, GM2]

Ceratobairdia wordensis (Hamilton, 1942) [Fig. 9K-R; GM1-GM3, GM5, GM6]

Ceratobairdia cf. ventrocostata Wang, 1978 [Fig. 11A, B; GM5, GM6]

Ceratobairdia pratti Forel sp. nov. [Fig. 11C-G; GM1, GM2]

Petasobairdia campbelli Chitnarin in Chitnarin et al., 2017 [Fig. 11H, I; GM2, GM4]

Petasobairdia cf. bicornuta Chen in Shi \& Chen, 2002 [Fig. 11J; GM5]

Petasobairdia? sp. [Fig. 11K; GM5]

Superfamily Sigillioidea Mandelstam, 1960

Family Microcheilinellidae Gramm, 1975

Microcheilinella postspinosa Chen, 1958 [Fig. 11L; GM5, GM6]

Microcheilinella sp. 15 in Zazzali et al. 2015 [Fig. 11M; GM5, GM6]

Microcheilinella sp. [Fig. 11N, O; GM5, GM6]

Denticupachydomella bellcanyonensis Forel sp. nov. [Fig. 11P, Q; GM5, GM6] 


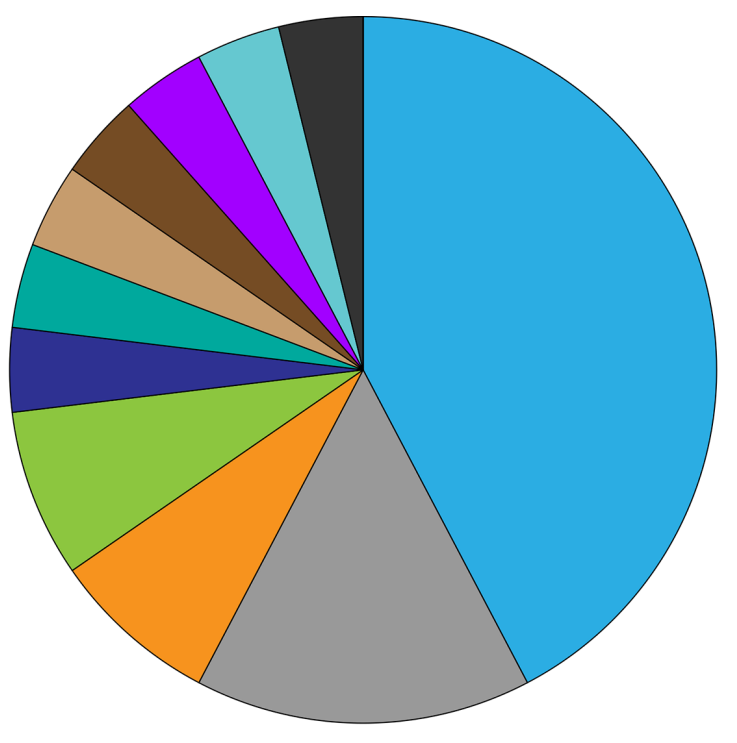

\section{Capitanian}

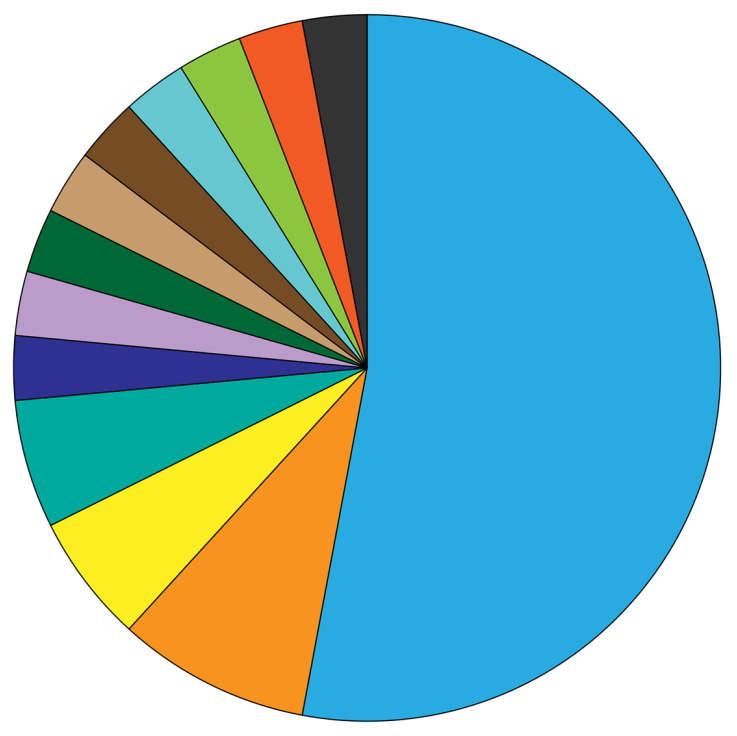

Roadian

\begin{tabular}{|ll|}
\hline$\square$ Bairdiidae & Scrobiculidae \\
$\square$ Kirkbyidae & $\square$ Hollinellidae \\
$\square$ Microcheilinidae & $\square$ Miltonellidae \\
$\square$ Amphissitidae & Glyptopleuridae \\
$\square$ Healdiidae & $\square$ Geisinidae \\
$\square$ Paraparchitidae & $\square$ Rozhdestvenskaytidae \\
$\square$ Cavellinidae & $\square$ Kirkbyellidae \\
$\square$ Kelletinidae & \\
\hline
\end{tabular}

Fig. 12. Circular diagrams of faunal composition of ostracod assemblages by number of species in the Roadian and Capitanian of the Guadalupe Mountains, West Texas, USA. 
(Roundyella), Hollinellidae (Hollinella), ?Miltonellidae (Libumella), Glyptopleuridae (Glyptopleura), Geisinidae (Geisina) and Rozhdestvenskaytidae (Fellerites), each being 3\% of the species. The Capitanian assemblage shows a slightly different structure with relatively diverse Microchellinellidae (Microcheilinella, Denticupachydomella) accounting for 15\% of the species. Secondary components are Kirkbyidae (Aurikirkbya, Kirkbyidae gen. et sp. indet.) and Paraparchitidae (Paraparchites) each with $8 \%$ of the species. Accessory families each account for less than $4 \%$ of the species: Healdiidae (Healdia), Cavellinidae (Sulcella), Kelletinidae (Kindlella), Scrobiculidae (Roundyella), Hollinellidae (Hollinella), Miltonellidae (Miltonella), Kirkbyellidae (Kirkbyella).

The autochthonous or allochthonous nature of ostracod assemblages can be assessed by considering the proportion of complete carapaces versus isolated valves and the demographic structure of populations (e.g., Oertli 1971; Boomer et al. 2003). As visible in all H/L scatter plots shown in Fig. 5 and Fig. 10, both assemblages are composed of a mixture of adults and juveniles, but very small juveniles are significantly lacking. Specimens were picked prior to this analysis and it is possible that only the largest specimens have been collected from the residues. In both assemblages, disarticulated valves are much more abundant than complete carapaces (Fig. 5 and Fig. 10), indicating that relatively important transportation occurred, in line with the lithological information pointing to deep-water downslope deposits in the Roadian Quarry section (Amerman 2009; Hurd \& Kerans 2014; Scholle et al. 2015). It is worth noting that none of the typical Palaeozoic off-shelf taxa (Beecherellidae Ulrich, 1894, Bythocytheridae Sars, 1866, Rectonariidae Gründel, 1962, Tricorninidae Blumenstengel, 1965) is present, which further confirms that these taxa may have been purely Palaeo-Tethyan in the Permian and the Triassic (Forel in press).

\section{Stratigraphical and palaeobiogeographical distributions}

\section{Species restricted to the USA}

Several of the species reported from the present analysis were already known from other North American localities. Several species previously known only from the Early Permian are herein extended to the Middle Permian:

- Kindlella fissiloba Sohn, 1954 previously only known from the Kungurian, Early Permian of Texas is herein shown to extend to the Roadian,

- Bairdia rhomboidalis Hamilton, 1942 documented from the Kungurian, Early Permian of Texas extends to the Capitanian,

- Aurikirkbya wordensis (Hamilton, 1942), Bairdia subfusiformis Hamilton, 1942 and Ceratobairdia wordensis (Hamilton, 1942) were only known from the Kungurian, Early Permian of Texas and are herein shown to occur in the Roadian and Capitanian,

- Bairdia radlerae Kellett, 1934 known from the Asselian and Sakmarian, Early Permian of Kansas herein is documented from the Roadian and Capitanian.

\section{Taxa with world wide distributions}

Of the species recognized in the Middle Permian of the Guadalupe Mountains, several have been previously documented outside of this area during the Permian (Fig. 13). The present work extends the stratigraphical range of Microcheilinella postspinosa that was only known from the Kungurian, to the Capitanian. Conversely, Acratia sinuata, Roundyella lebaensis, Kindlella bellsundi and Microcheilinella sp. 15 in Zazzali et al. 2015 were only known from the Changhsingian, Late Permian, worldwide and herein are shown to have radiated in the Middle Permian. It is important to mention that stratigraphical updates are also observed at the generic level: until now Denticupachydomella was only known from the Late Permian but we show that it radiated in the Capitanian. 
In terms of palaeobiogeographical distribution of taxa through time, several patterns are also visible. The species Sulcella mesopermiana, Petasobairdia campbelli, Fabalicypris glennensis and Microcheilinella postspinosa had a purely Palaeo-Tethyan distribution until the present contribution but their occurrence

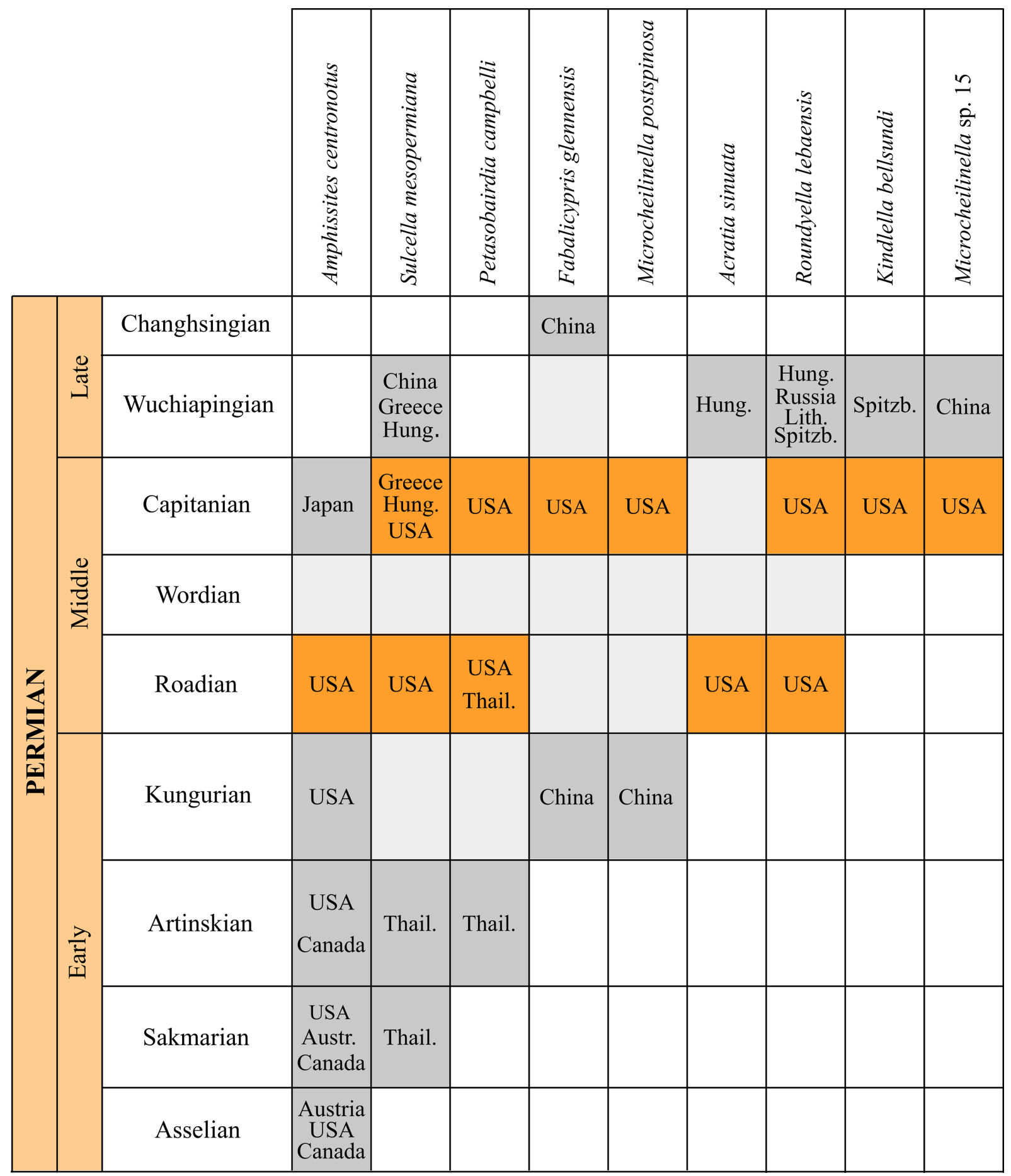

Fig. 13. Stratigraphical (Permian only) and palaeogeographic ranges of ostracod species recorded outside of the USA in the Permian; stage durations not to scale. Abbreviations: Hung. = Hungary; Spitzb. =Spitzbergen.; Lith. =Lithuania; Thail. =Thailand; Austr. =Australia. Orange colour underlined = species found in this study; light grey = supposed occurences; dark grey $=$ ocurrences of species in common with this study. 
in the Middle Permian of the Guadalupe Mountains documents a transpanthalassic dispersal of species in the Permian. In the present state of our knowledge, these species all appear to have radiated on the eastern margin of Palaeo-Tethys sensu lato in the Early Permian (Fig. 13), but their dispersal patterns show significant discrepancies. Sulcella mesopermiana was restricted to the Indochina Block, Thailand (Chitnarin et al. 2017) and later spread westward to the eastern Palaeo-Tethys (Greece, Hungary) and eastward across the Panthalassa to the Guadalupe Mountains area in the Middle Permian. Conversely, Petasobairdia campbelli, Fabalicypris glennensis and Microcheilinella postspinosa only record a transpanthalassic eastward migration trend, but this feature may change with more data from diverse localities worldwide. A second group of species documents the opposite trend: Acratia sinuata, Roundyella lebaensis, Kindlella bellsundi and Microcheilinella sp. 15 in Zazzali et al. 2015 radiated in the Middle Permian of the Guadalupe Mountains area as revealed by the present assemblages and migrated westward to the Palaeo-Tethyan area in the Late Permian. These two migration ways herein illustrated for the first time show that exchanges of taxa between Palaeo-Tethys sensu lato and Panthalassa were strong in the Permian prior to the end-Permian extinction. The strong relationship of the northern America ostracod assemblages with the eastern Tethyan area, more precisely the South China Block, was already reported in Forel (2018). A second observation is that the eastern Palaeo-Tethys has been a zone of major importance in radiation of ostracod taxa in the Permian, as it was in the Triassic (Bate 1977; Kristan-Tollmann 1983, 1993; Lord 1988; Ketmuangmoon et al. 2018; Forel et al. 2019; Forel \& Moix 2020).

\section{Conclusion}

Ostracods of Roadian and Capitanian (Middle Permian) age have been extracted from samples collected from the Quarry and MKCS1 sections in the Guadalupe Mountains area in West Texas, USA. In total, the ostracod assemblages are composed of 49 species, which represent 26 genera and 15 families. Eleven new species are described: Healdia mckittrickensis Crasquin sp. nov., Healdia cutoffella Crasquin sp. nov., Aurikirkbya guadalupensis Crasquin sp. nov., Hollinella (Hollinella) williamsranchensis Crasquin sp. nov., Geisina culbersonensis Crasquin sp. nov., Paraparchites pecosensis Crasquin sp. nov., Bairdia elcapitanensis Forel sp. nov., Ceratobairdia mescaleroella Forel sp. nov., Ceratobairdia sexagintaduella Forel sp. nov., Ceratobairdia pratti Forel sp. nov., and Denticupachydomella bellcanyonensis Forel sp. nov. The Roadian and Capitanian assemblages are largely dominated by isolated valves that document transportation, in line with other proxies indicating deep-water downslope deposition. The stratigraphical and palaeobiogeographic distribution of taxa indicate transpanthalassic exchanges of taxa in the Permian as well as the importance of the eastern Palaeo-Tethys margin for radiation of ostracod species.

\section{Acknowledgements}

We are grateful to the Muséum national d'histoire naturelle for the master grant (AAP) provided to the first author of this work. We thank Gorden Bell, former Guadalupe Mountains National Park geologist, for access to the MKCS1 locality. Alexandre Lethiers (Sorbonne Universite, Paris, France) is acknowledged for his precious help with the figures as well as two anonymous reviewers who provided comments that improved an earlier version of this manuscript.

\section{References}

Amerman R. 2009. Deepwater Mass-Transport Deposits; Structure, Stratigraphy, and Implications for Basin Evolution. PhD thesis, Colorado School of Mines, Golden.

Bate R.H. 1977. Jurassic Ostracoda of the Atlantic Basin. In: Swain F.M. (ed.) Stratigraphic Micropaleontology of Atlantic Basin and Borderlands: 231-244. Elsevier Scientific Publications, New York, Amsterdam. https://doi.org/10.1016/s0920-5446(08)70354-6 
Becker G. 1997. The superfamily Kirkbyacea Ulrich \& Bassler, 1906. 2. Family Amphissitidae Knight, 1928. Contributions to Palaeozoic Ostracod Classification [POC] 3. Neues Jahrbuch für Geologie und Paläontologie, Abhandlungen 204: 127-140. https://doi.org/10.1127/njgpa/204/1997/127

Becker G. 2001. The Superfamily Bairdiacea Sars, 1888-1. Family Bairdiidae Sars, 1888 (Palaeozoic members only). Contributions to Palaeozoic Ostracod Classification [POC] 18. Neues Jahrbuch für Geologie und Paläontologie, Abhandlungen 220 (2): 267-294.

https://doi.org/10.1127/njgpa/220/2001/267

Becker G. \& Wang S.Q. 1992. Kirkbyacea and Bairdiacea (Ostracoda) from the Palaeozoic of China. Palaeontographica Abteilung A 224: 1-54.

Boomer I., Horne D.J. \& Slipper I.J. 2003. The use of ostracods in palaeoenvironmental studies or what can you do with an ostracod shell? Palaeontological Society Papers 9: 153-179.

https://doi.org/10.1017/s1089332600002199

Bradfield H.H. 1935. Pennsylvanian Ostracoda of Ardmore Basin, Oklahoma. Bulletins of American Paleontology 22 (73): 1-145.

Burke C.D. 1985. Paleoecologic interpretation of Ostracoda from Skelley Member, Conemaugh (Pennsylvanian), southeastern Ohio. Journal of Paleontology 19: 839-848.

Chen T.C. 1958. Permian ostracods from the Chihsia Limestone of Lungtan, Nanking. Acta Palaeontologica Sinica 6: 235-257.

Chen D.Q. \& Bao H. 1986. Lower Permian Ostracodes from the Chihsia Formation of Jurongand Longtar, Jiangsu Province. Acta Micropalaeontologica Sinica 3: 107-132.

Chitnarin A., Crasquin S., Charoentitirat T., Tepnarong P. \& Thanee N. 2012. Ostracods (Crustacea) of the Early-Middle Permian from Central Thailand (Indochina block). Part 1. Order Palaeocopida. Geodiversitas 34 (4): 801-835. https://doi.org/10.5252/g2012n4a5

Chitnarin A., Crasquin S., Forel M.-B. \& Tepnarong P. 2017. Ostracods (Crustacea) of the EarlyMiddle Permian (Cisarulian-Guadalupian) from Central Thailand (Indochina Block): Part II, Orders Podocopida, Platycopida and Myodocopida. Geodiversitas 39 (4): 651-690.

https://doi.org/10.5252/g2017n4a1

Christopher C.C. Jr., Hoare R.D. \& Sturgeon M.T. 1990. Pennsylvanian Hollinacean and Kirkbyacean Ostracodes from the Appalachian Basin. Journal of Paleontology 64 (6): 967-987.

https://doi.org/10.1017/s0022336000020047

Cooper C.L. 1946. Pennsylvanian ostracodes of Illinois. Bulletin State Geological Survey of Illinois 70: $1-177$.

Cooper G.A. \& Grant R.E. 1964. New Permian stratigraphic units in Glass Mountains, west Texas. Bulletin of the American Association of Petroleum Geologists 48 (9): 1581-1588.

https://doi.org/10.1306/BC743DA3-16BE-11D7-8645000102C1865D

Coryell H.N. \& Billings G.D. 1932. Pennsylvanian Ostracoda of the Wayland Shale of Texas. American Midland Naturalist 13 (4): 170-189. https://doi.org/10.2307/2420267

Crasquin S. 1985. Zonation par les ostracodes dans le Mississippien de l'Ouest canadien. Revue de Paléobiologie 4 (1): 43-52.

Crasquin S. \& Forel M.-B. 2014. Ostracods (Crustacea) through Permian-Triassic events. Earth Science Reviews 137: 52-64. https://doi.org/10.1016/j.earscirev.2013.01.006 
Crasquin S., Forel M.-B., Feng Q.L., Yuan A.H., Baudin F. \& Collin P.-Y. 2010. Ostracods (Crustacea) through the Permian-Triassic boundary in South China: The Meishan stratotype (Zhejiang Province). Journal of Systematic Palaeontology 8: 331-370. https://doi.org/10.1080/14772011003784992

Crasquin S., Forel M.-B., Yuan A.H., Nestell G. \& Nestell M. 2018. Species of Hollinella (Palaeocopida: Ostracoda: Crustacea) as stratigraphical indices of the Late Permian-Early Triassic post-extinction interval. Journal of Systematic Palaeontology 16 (3): 213-224.

https://doi.org/10.1080/14772019.2017.1283648

Crasquin-Soleau S. 1997. First Upper Paleozoic ostracodes from British Columbia (Canada): Harper Ranch Group. Palaeontographica Abteilung A 244: 37-84.

Crasquin-Soleau S. \& Baud A. 1998. New Permian ostracods from Greece (Hydra Island). Journal of Micropalaeontology 17: 131-152. https://doi.org/10.1144/jm.17.2.131

Crasquin-Soleau S. \& Orchard M.J. 1994. Upper Paleozoic ostracodes of the Harper Ranch beds (southcentral British Columbia, Canada). Micropaleontology 40: 242-254. https://doi.org/10.2307/1485818

Crasquin-Soleau S., Broutin J., Roger J., Platel J.P., Al Hashmi H., Angiolini L., Baud A., Bucher H. \& Marcoux J. 1999. First Permian ostracode fauna from the Arabian Plate (Khuff Formation, Sultanate of Oman). Micropaleontology 45 (2): 163-182. https://doi.org/10.2307/1486111

Crasquin-Soleau S., Marcoux J., Angiolini L., Richoz S., Nicora A., Baud A. \& Bertho Y. 2004. A new ostracode fauna from the Permian-Triassic boundary in Turkey (Taurus, Antalya Nappes). Micropaleontology 50 (3): 281-295. https://doi.org/10.2113/50.3.281

Delo D.M. 1930. Some Upper Carboniferous Ostracoda from the shale basin of western Texas. Journal of Paleontology 4: 152-178.

Ferdinando D. 2001. Ostracode and Foraminiferal Taxonomy and Paleoecology of the Holmwood Shale, Northern Perth Basin, Western Australia. PhD thesis, The University of Western Australia.

Fohrer B. 1991.Verkieselte Flachwasser-Ostracoden und ihre Begleitfauna aus dem Oberkarbon der Karnischen Alpen (Nassfeld-Region,Kärnten, Österreich). Abhandlungen der Geologischen Bundesanstalt 46: 1-107.

Fohrer B. 1997. Ostracoden aus dem Oberkarbon und Unterperm der Kärnischen Alpen (Österreich): Systematik, Biostratigraphie und Palökologie. Jahrbuch der Geologischen Bundesanstalt 140: 99-191.

Forel M.-B. 2018. Rediscovering the Lys collection in the Muséum national d'histoire naturelle (Paris, France): first report on Middle Permian ostracods from occidental Hindu Kush, Afghanistan. Geodiversitas 40 (18): 471-496. https://doi.org/10.5252/geodiversitas2018v40a18

Forel M.-B. in press. Thoughts on the Late Paleozoic-Early Mesozoic records of deep-sea ostracods. Micropaleontology.

Forel M.-B. \& Crasquin S. 2020. Bounded by crises: an updated view of the evolution of marine ostracods during the Triassic. Marine Micropaleontology: e101925.

https://doi.org/10.1016/j.marmicro.2020.101925

Forel M.-B. \& Grădinaru E. 2020. Rhaetian (Late Triassic) ostracods from the offshore prolongation of North Dobrogean Orogen into the Romanian Black Sea Shelf. European Journal of Taxonomy 727: 1-83. https://doi.org/10.5852/ejt.2020.727.1183

Forel M.-B. \& Moix P. 2020. Late Triassic ostracods from the Lycian Nappes, southwestern Turkey: implications on taxonomy and palaeobiogeographical distribution. Geodiversitas 191 (30): 1-18. https://doi.org/10.1051/bsgf/2020035 
Forel M.-B., Thuy B. \& Wisshak M. 2019. Digging into the ancestral stocks of Jurassic lineages: Ostracods (Crustacea) from Carnian (Late Triassic) sponge mounds from the Maantang Formation (South China). Bulletin de la Société géologique de France 190 (9): 1-28. https://doi.org/10.1051/bsgf/2019009

Geis H.L. 1932. Some ostracodes from the Salem Lime- stone, Mississippian of Indiana. Journal of Paleontology 6: 149-188. Available from https://www.jstor.org/stable/1298173 [accessed 2 Aug. 2021].

Girty G.H. 1908. The Guadalupian Fauna. United States Geological Survey, Professional Paper 58: 1-652. https://doi.org/10.3133/pp58

Girty G.H. 1910. The fauna of the Phosphate Beds of the Park City Formation in Idaho, Wyoming, and Utah. United States Geological Survey Bulletin 436: 1-82. https://doi.org/10.3133/b436

Glenister B.F., Wardlaw B.R., Lambert L.L., Spinosa C., Bowring S.A., Erwin D.H., Menning M. \& Wilde G.L. 1999. Proposal of Guadalupian and component Roadian, Wordian and Capitanian Stages as International standards for the Middle Permian Series. Permophiles 34: 3-11.

Gramm M.N. 1997. Ostracodes of the Chandalaz horizon (Upper Permian) of south Primorye. Mémoires de Géologie (Lausanne) 30: 61-66.

Green R. 1963. Lower Mississippian ostracodes from the Banff Formation, Alberta. Research Council of Alberta, Bulletin 11: 1-237.

Hamilton B. 1942. Ostracodes from the Upper Permian of Texas. Journal of Paleontology 16 (6): 712 718.

Harlton B.H. 1927. Some Pennsylvanian Ostracoda of the Glenn and Hoxbar Formations of southern Oklahoma and of the upper part of the Cisco Formation of northern Texas. Journal of Paleontology 1 (3): 203-212.

Harlton B.H. 1933. Micropaleontology of the Pennsylvanian Johns Valley Shale of Ouachita Mountains, Oklahoma and its relationships to the Mississippian Caney Shale. Journal of Paleontology 7: 3-29.

Harris M.T. 2000. Members for the Cutoff Formation, Western Escarpment of the Guadalupe Mountains, West Texas. In: Wardlaw B.R., Grant R.E. \& Rohr D.M. (eds) The Guadalupian Symposium. Smithsonian Contributions to the Earth Sciences 32: 101-120.

Hoare R.D. \& Mapes R.H. 2000. Ostracodes from the Imo Formation (Mississippian, Chesterian) of North Central Arkansas. Journal of Paleontology 74 (52): 1-23. https://doi.org/dm5xg3

Hoare R.D. \& Merrill G.K. 2004. A Pennsylvanian (Morrowan) ostracode fauna from Texas. Journal of Paleontology 78 (1): 185-204. https://doi.org/c63z5x

Hoare R.D., Svitko J.L. \& Sturgeon M.T. 1999. Pennsylvanian Bairdiidae (Ostracoda) from the Appalachian Basin. Journal of Paleontology 73 (3): 437-453. https://doi.org/10.1017/s0022336000027967

Horne D.J., Cohen A. \& Martens K. 2002. Taxonomy, morphology and biology of Quaternary and living Ostracoda. American Geophysical Union, Washington (Geophysical Monograph Series) 131: 5-36. https://doi.org/10.1029/131gm02

Hurd G. \& Kerans C. 2014. Advances in the stratigraphic model of the Cutoff Formation with implications for Upper Bone Spring and Avalon Reservoirs: Western Escarpment, Guadalupe Mountains. West Texas Geological Society Field Trip Guidebook: 14-129.

Hurd G., Kerans C., Fullmer S. \& Janson X. 2016. Large-scale inflections in slope angle below the shelf break: a first order control on the stratigraphic architecture of carbonate slopes, Cutoff Formation, Guadalupe Mountains National Park, West Texas. Journal of Sedimentary Research 86: 336-362. https://doi.org/10.2110/jsr.2016.25 
Hurd G., Kerans C., Fullmer S. \& Janson X. 2018. New members of the Cutoff Formation in the southern Guadalupe Mountains near the Global Stratotype Section and Point for the base of the Roadian Stage, Texas. Stratigraphy 15 (2): 109-133. https://doi.org/10.29041/strat.15.2.109-133

Ishizaki K. 1964. Middle Permian ostracodes from the Iwaizaki limestone, northeast Japan. The Science Reports of the Tohoku University. Second Series, Geology 36 (1): 139-151.

Ivanov A.O., Nestell M.K., Nestell G.P. \& Bell Jr G.L. 2020. New fish assemblages from the Middle Permian from the Guadalupe Mountains, West Texas, USA. Palaeoworld 29: 239-256.

https://doi.org/10.1016/j.palwor.2018.10.003

Ivanov V.K. 1975. Остракоды. In: П. И. Сувейздис (ed.) Пермская система Прибалтики: 156-184. Вильнюс. [In Russian.]

Johnson W.R. 1936. The ostracods of the Missouri Series of Nebraska. Nebraska Geological Survey Paper 11: 1-52.

Jones P.J. 1988. Carapace sculpture in Amphissites (Kirkbyacea: Ostracoda). In: Hanai T., Ikeya N. \& Ishizaki K. (eds) Evolutionary Biology of Ostracoda, Developments in Paleontology and Stratigraphy 11: 259-270. Elsevier, Amsterdam. https://doi.org/10.1016/S0920-5446(08)70188-2

Jones P.J. 1989. Lower Carboniferous Ostracoda (Beyrichicopida and Kirkbyocopa) from the Bonaparte Basin, North-Western Autralia. Australian Bureau of Mineral Ressources, Australia Bulletin 228: 1-97.

Jones T.R. 1859. [With notes on the species, in] On Permian Entomostraca from the shell-limestone of Durham. Transactions of the Tyneside Naturalists' Field Club 4: 133-137.

Kellett B. 1933. Ostracodes of the Upper Pennsylvanian and the Lower Permian strata of Kansas: I. the Aparchitidae, Beyrichiidae, Glyptopleuridae, Kloedenellidae, Kirkbyiidae, and Youngiellidae. Journal of Paleontology 7 (1): 59-108.

Kellett B. 1934. Ostracodes of the Upper Pennsylvanian and the Lower Permian Strata of Kansas: II. The Genus Bairdia. Journal of Paleontology 8: 120-138.

Kellett B. 1935. Ostracodes of the Upper Pennsylvanian and the Lower Permian strata of Kansas: III. Bairdiidae (concluded), Cytherellidae, Cypridinidae, Entomoconchidae, Cytheridae and Cypridae. Journal of Paleontology 9: 132-166.

Ketmuangmoon P., Chitnarin A., Forel M.-B. \& Tepnarong P. 2018. Diversity and paleoenvironmental significance of Middle Triassic ostracods (Crustacea) from northern Thailand: Pha Kan Formation (Anisian, Lampang Group). Revue de Micropaléontologie 61 (1): 3-22.

https://doi.org/10.1016/j.revmic.2017.11.001

Khivintseva E.A. 1969. New ostracods from the Kazanian deposits of Orenburg Province. Paleontological Journal 1: 85-94.

Knight J.B. 1928. Some Pennsylvanian Ostracodes from the Henrietta Formation of Eastern Missouri (part 1). Journal of Paleontology 3: 229-267.

Knüpfer J. 1967. Mikrofauna aus dem unteren Teil des Zechsteins von Rügen. Freiberger Forschungshefte C213: 73-87.

Kotschetkova N.M. 1959. Ostracody nizhnekazanskogo podyarysa zapadnoi Bashkirii [Ostracods of the Lower Kazanian Stage of Western Bashkiria]. In: Olli A.I. (ed.) Voprosy geologii vostochnoi Ola-ainy Russkoi Platformy i Yuzhnogo Urala [Problems in the Geology of the Eastern Borders of the Russian Platform and the South Urals]: 5-28. Akademiya Nauk SSSR, Bashkirskii Filial, Gorno-Geologicheskii Institut, Ufa. 
Kozur H. 1991. Neue Ostracoden - Arten aus dem Oberen Mittelkarbon (Höberes Moskovian), Mittelund Oberperm des Bükk-gebirges (N-Ungarn). Geologisch- Paläontologische Mitteilungen Innsbruck 2: $1-145$.

Kozur H.W., Aydin M., Demir O., Yakar H., Göncüoglu M.C. \& Kuru F. 2000. New stratigraphic and palaeogeographic results from the Palaeozoic and Early Mesozoic of the Middle Pontides (Northern Turkey) in the Azdavay, Devrekani, Küre and Inebolu areas: implications for the Carboniferous - Early Cretaceous geodynamic evolution and some related remarks to the Karakaya Oceanic Rift Basin. Geologica Croatica 53 (2): 209-268.

Kristan-Tollmann E. 1983. Ostracoden aus dem Oberanis von Leidapo bei Guiyang in Südchina. Neue Beiträge zur Biostratigraphie der Tethys-Trias 5: 121-176.

Kristan-Tollmann E. 1993. Zur paläogeographischen Verbreitung der Ostracoden-Gattung Hermiella an der Rhät/Lias-Grenze. Zitteliana 20: 331-342.

Krömmelbein K. 1958. Ostracoden aus dem unteren Zechstein der Bohrung Leba in Pommern. Geologisches Jahrbuch 75: 115-135.

Lambert L.L., Lehrmann D.J. \& Harris M.T. 2000. Correlation of the Road Canyon and Cutoff Formations, West Texas, and its relevance to establishing an International Middle Permian (Guadalupian) Series. In: Wardlaw B.R., Grant R.E. \& Rohr D.M. (eds) The Guadalupian Symposium. Smithsonian Contributions to the Earth Sciences 32: 153-184.

Lord A.R. 1988. Ostracoda of the Early Jurassic Tethyan Ocean. In: Hanai T., Ikeya N. \& Ishizaki K. (eds) Evolutionary Biology of Ostracoda: Its Fundamentals and Applications: 855-868. Proceedings of the Ninth International Symposium on Ostracoda, Shizuoka, Japan, 1985. Developments in Palaeontology and Stratigraphy 11. Kodansha, Tokyo and Elsevier, Amsterdam.

https://doi.org/10.1016/s0920-5446(08)70226-7

Melnyk D.H. \& Maddocks R.F. 1988. Ostracode biostratigraphy of the Permo-Carboniferous of central and north-central Texas, Part I: paleoenvironmental framework. Micropaleontology 34: 1-20.

https://doi.org/10.2307/1485607

Moore R.C. 1961. Treatise on Invertebrate Paleontology. Arthropoda 3, Crustacea, Ostracoda. Geological Society of America and University of Kansas Press.

Nestell G.P. \& Nestell M.K. 2020. Roadian (earliest Guadalupian, Middle Permian) Radiolarians from the Guadalupe Mountains, West Texas, USA Part I: Albaillellaria and Entactinaria. Micropaleontology 66 (1): $1-50$.

Nestell M.K., Nestell G.P., Wardlaw B.R. \& Ivanov A.O. 2015. A diverse Roadian (Guadalupian, Middle Permian) microfauna from the Guadalupe Mountains, West Texas, USA. In: Nurgaliev D.K. (eds) XVIII International Congress on the Carboniferous and Permian. Abstract Volume: 134. Kazan.

Oertli H.J. 1971. The aspect of ostracode fauna - a possible new tool in petroleum sedimentology. In: Oertli H.J. (ed.) Paléoécologie des Ostracodes: 137-151. Bulletin du Centre de Recherches de Pau, SNPA, Pau.

Olempska E. 1999. Silicified shallow-water ostracodes from the Early Carboniferous of South China. Acta Palaeontologica Polonica 44 (4): 383-436.

Olempska E. \& Błaszyk J. 1996. Ostracods from Permian of Spitsbergen. Polish Polar Research 17 (1-2): 3-20.

Salas M.J., Vannier J. \& Williams M. 2007. Early Ordovician ostracods from Argentina: their bearing on the origin of binodicope and palaeocope clades. Journal of Paleontology 81 (6): 1384-1395.

https://doi.org/10.1666/05-134.1 
Sanchez de Posada L.C. 1977. Ostracodes from the Upper Carboniferous of La Camocha Coal Mine (Asturias, N. Spain). Revista Española de Micropaleontología 9: 411-438.

Schneider G.F. 1966. Upper Permian ostracods of the Russian Platform and their importance for stratigraphy. In: Vyalov O.S. (ed.) Fossil Ostracoda: 109-125. Papers from the First All-Union Symposium on Fossil Ostracods, Lvov, 1963. Izdatel'stvo "Naukova Dumka”, Kiev [Israel Program for Scientific Translations, Jerusalem, 1971].

Scholle P., Ulmer-Scholle D., Nestell M. \& Nestell G. 2015. Around the Horn. Guadalupian age basinal facies in the western Delaware Mountain Group Outcrop Belt, Guadalupe and Apache Mountains. In: Trentham R. (ed.) West Texas Geological Society, WTGS Annual Fall Field Trip, Publication 15-131. West Texas Geological Society, Midland, Texas.

Scott H.W. 1959. The type species of Paraparchites Ulrich \& Bassler. Journal of Paleontology 33: $670-674$.

Shaver R.H. \& Smith S.G. 1974. Some Pennsylvanian ostracods from Lawrence County, Illinois. Journal of Paleontology 15: 354-358.

Shen S.Z., Yuan D.X., Henderson C.M., Wu Q., Zhang Y.C., Zhang H., Mu L., Ramezani J., Wang X.D., Lambert L.L., Erin D.H., Hearst J.M., Xiang L., Chen B., Fan J.X., Wang Y., Wang Q., Qi Y.P., Qie W.K. \& Wang T.T. 2020. Progress, problems and prospects: An overview of the Guadalupian Series of South China and North America. Earth-Science Reviews 211: 103412.

https://doi.org/10.1016/j.earscirev.2020.103412

Shi C.G. 1982. Some Early Carboniferous ostracodes from Nylam, Xizang (Tibet). Acta Palaeontologica Sinica 21: 309-314.

Shi C.G. \& Chen D.Q. 2002. Late Permian ostracodes from Heshan and Yishan of Guangxi. Bulletin of the Nanjing Institute Geology and Paleontology 15: 47-129. [In Chinese with English abstract.]

Sohn I.G. 1950. Growth series of ostracodes from the Permian of Texas. United States Geological Survey Professional Paper 221-C: 33-43. https://doi.org/10.3133/pp221c

Sohn I.G. 1954. Ostracoda from the Permian of the Glass Mountains, Texas. United States Geological Survey Professional Paper 264-A: 1-24. https://doi.org/10.3133/pp264a

Sohn I.G. 1960. Paleozoic species of Bairdia and related genera. United States Geological Survey Professional Paper 330-A: 1-105. https://doi.org/10.3133/pp330a

Sohn I.G. 1961a. Ostracodes and conodonts from the Getaway limestone member of the Cherry Canyon formation (Permian), Texas. Geological Survey Research 1961. Short Papers in the Geologic and Hydrologic Sciences, Articles 293-435: D244-D245.

Sohn I.G. 1961b. Aechminella, Amphissites, Kirkbyella and related genera. United States Geological Survey Professional Paper 330-B: 107-160. https://doi.org/10.3133/pp330b

Sohn I.G. 1962. Stratigraphic significance of the Paleozoic ostracode genus Coryellina Bradfield, 1935. Journal of Paleontology 36 (6): 1201-1213.

Sohn I.G. 1971. New Late Mississippian Ostracode genera and species from Northern Alaska. A revision of the Paraparchitacea. United States Geological Survey Professional Paper 711A: 1-24.

https://doi.org/10.3133/pp711a

Sohn I.G. 1972. Late Paleozoic Ostracode Species from the conterminous Unites States. A revision of the Paraparchitacea. Unites States Geological Survey Professional Paper 711-B: B1-B13.

https://doi.org/10.3133/pp711B 
Sohn I.G. 1982. Biostratigraphic evaluation of the Carboniferous and Permian ostracodes of Texas: 103-109. In: Maddocks R.F. (ed.) Texas Ostracoda. Guidebook of Excursions and related papers for the Eighth International Symposium on Ostracoda, July 24-25 and July 30 - August 3, 1982. University of Houston, Texas.

Spinosa C., Furnish W.M. \& Glenister B.F. 1975. The Xenodiscidae, Permian ceratitoid ammonoids. Journal of Paleontology 49 (2): 239-283.

Tanaka G., Ono T., Yuan A.H., Ichida M. \& Maeda H. 2012. Early Permian ostracods from Mugi County, Gifu Prefecture, Central Japan. Paleontological Research 16: 88-106.

https://doi.org/10.2517/1342-8144-16.2.088

Tanaka G., Ono T., Nishimura T. \& Maeda H. 2013. Middle Permian ostracods from the Akasaka Limestone, Gifu Prefecture, central Japan. Paleontological Research 16: 289-306.

https://doi.org/10.2517/1342-8144-16.4.289

Thompson M.L. \& Shaver R.H. 1964. Early Pennsylvanian microfaunas of the Illinois Basin. Illinois Academy of Sciences Transactions 57: 3-23.

Ulrich E.O. \& Bassler R.S. 1906. New American Palaeozoic Ostracoda. Notes and descriptions of the Upper Carboniferous genera and species. United States National Museum Paper 30: 149-164.

https://doi.org/10.5479/si.00963801.30-1446.149

Ulrich E.O. \& Bassler R.S. 1923. Paleozoic Ostracoda: their morphology, classification and occurence. In: Maryland Geological Survey (ed.) Silurian: 271-391. Johns Hopkins Press, Baltimore.

Wang S.Q. 1978. Late Permian and Early Triassic ostracods of Western Guizhou and Northeastern Yunnan. Acta Palaeontologica Sinica 17: 277-308.

Wardlaw B.R. 2002. The upper Roadian Willis Ranch Member of the Word Formation is the Third Limestone Member of the Word Formation of King (1931). Permophiles 40: 32.

Wardlaw B.R. 2004. Guadalupian News. Permophiles 44: 18.

Woszczyńska S. 1987. Foraminifera and ostracods from the carbonate sediments of the polish Zechstein. Acta Palaeontologica Polonica 32 (3-4): 155-205.

Yi W.J. 1993. Ostracode fauna from lower Permian Chihsia Formation of Guangping in Datian, Fujian. Acta Palaeontologica Sinica 32: 561-586.

Yi W.J. 2004. Ostracodes from the Upper Permian and Lower Triassic at the Kongtongshan section of Datian, Fujian. Acta Palaeontologica Sinica 43: 556-570.

Yuan A., Crasquin-Soleau S., Feng Q.L. \& Gu S. 2007. Latest Permian deep-water ostracods from southwestern Guangxi, South China. Journal of Micropalaeontology 26 (2): 169-191.

https://doi.org/10.1144/jm.26.2.169

Yuan A., Crasquin-Soleau S., Feng Q.L. \& Gu S. 2009. Ostracods from uppermost Permian siliceous and muddy rocks of Guizhou, Guangxi and Anhui. Acta Micropalaeontologica Sinica 26: 385-403.

Zazzali S. 2016. Paléobiodiversité des ostracodes à la limite Permien moyen - Permien supérieur. PhD Thesis, Université Paris 6, Golden.

Zazzali S., Crasquin S., Deconinck J.F. \& Feng Q.L. 2015. Biodiversity across the GuadalupianLopingian Boundary: first results on the ostracod (Crustacea) fauna, Chaotian section (Sichuan Province, South China). Geodiversitas 37 (3): 283-313. https://doi.org/10.5252/g2015n3a1

Zhang X.J. \& Liang X.Y. 1987. Ostracoda from the Taiyuan Formation of Xingyang and Gong Xian districts, Henan. Acta Micropalaeontologica Sinica 4: 293-312. 
Manuscript received: 23 March 2021

Manuscript accepted: 16 July 2021

Published on: 22 September 2021

Topic editor: Christian de Muizon

Desk editor: Kristiaan Hoedemakers

Printed versions of all papers are also deposited in the libraries of the institutes that are members of the EJT consortium: Muséum national d'histoire naturelle, Paris, France; Meise Botanic Garden, Belgium; Royal Museum for Central Africa, Tervuren, Belgium; Royal Belgian Institute of Natural Sciences, Brussels, Belgium; Natural History Museum of Denmark, Copenhagen, Denmark; Naturalis Biodiversity Center, Leiden, the Netherlands; Museo Nacional de Ciencias Naturales-CSIC, Madrid, Spain; Real Jardín Botánico de Madrid CSIC, Spain; Zoological Research Museum Alexander Koenig, Bonn, Germany; National Museum, Prague, Czech Republic. 